\title{
LOCAL ESTIMATE OF FUNDAMENTAL GROUPS
}

\author{
GUOYI XU
}

\begin{abstract}
Aвstract. For any complete $n$-dim Riemannian manifold $M^{n}$ with nonnegative Ricci curvature, Kapovitch and Wilking proved that any finitely generated subgroup of the fundamental group $\pi_{1}\left(M^{n}\right)$ can be generated by $C(n)$ generators. Inspired by their work, we give a quantitative proof of the above theorem and show that $C(n) \leq n^{n^{20 n}}$. Our main tools are quantitative Cheeger-Colding's almost splitting theory, and the squeeze lemma for covering groups between two Riemannian manifolds with nonnegative Ricci curvature.
\end{abstract}

\section{CONTENTS}

Introduction . . . . . . . . . . . . . . . . . . . . . . . . 1

Part I. G-H approximation yields A.O.L. harmonic functions . . . . . . 5

1. Almost orthonormal local Busemann functions. . . . . . . . . . 6

2. Existence of almost orthonormal linear (A.O.L.) harmonic functions . . 18 Part II. A.O.L. harmonic functions produce G-H approximation . . . . . 32

3. Existence of almost linear function and Hessian estimate . . . . . . 32

4. Segment inequality and measure of 'good' points . . . . . . . . . . 37

5. Quantitative almost splitting theorem . . . . . . . . . . . . . 42

Part III. Covering groups of Riemannian manifolds with $R c \geq 0$. . . . . 53

6. Squeeze Lemma and Dimension induction on harmonic functions . . . 53

Acknowledgments . . . . . . . . . . . . . . . . . . . . . 62

References . . . . . . . . . . . . . . . . . . . . 62

\section{INTRODUCTION}

It is well-known that any compact Riemannian manifold $\left(M^{n}, g\right)$ has finitely generated fundamental group. For non-compact complete Riemannian manifolds, the conclusion is not always right. For example, the surface with infinite genus has infinitely generated fundamental group. A natural question is:

Question 0.1. For which complete Riemannian manifold $\left(M^{n}, g\right)$, the fundamental group $\pi_{1}\left(M^{n}\right)$ is finitely generated?

Date: June 3, 2019.

The author was partially supported by NSFC-11771230. 
Note the above example has no non-negative sectional curvature metric, to obtain the finitely generatedness of fundamental group, we may consider adding some curvature assumption on complete Riemannian manifolds.

For the group $\mathbf{G}$, we define that $\mathfrak{n g}(\mathbf{G})$ is the minimal number of generators needed. In 1911 Bieberbach proved: For complete flat Riemannian manifold $M^{n}$, the fundamental group $\pi_{1}\left(M^{n}\right)$ is finitely generated and $\mathfrak{n g}\left(\pi_{1}\left(M^{n}\right)\right) \leq C(n)$.

Bieberbach reduced the study of fundamental groups of flat manifolds to the study of the discrete subgroup of the isometry group of $\mathbb{R}^{n}$, which was later developed into a more general theory about the discrete subgroups of Lie groups (see [Rag72]).

Later for hyperbolic manifolds, the following theorem was obtained (see [BGS85]): Any finite volume hyperbolic manifold has finitely generated fundamental group.

Remark 0.2. The above theorem is a corollary of the following general result in Lie group theory: Any lattice $\Gamma$ of a Lie group $\mathbf{G}$ is finitely generated, where lattice means that $\Gamma$ is a discrete subgroup of $\mathbf{G}$ and $\mathbf{G} / \Gamma$ has finite volume.

The study of the above non-positive curvature case, has more algebraic flavor, which has strong contrast to the following non-negative curvature case.

In 1972, Cheeger and Gromoll [CG72] studied non-negative sectional curvature Riemannian manifolds. Among other things, they obtained the following result: If $M^{n}$ has sectional curvature sec $\geq 0$, then $\pi_{1}\left(M^{n}\right)$ is finitely generated.

In fact, in [CG72] it was showed that $M^{n}$ is homotopic to a compact totally geodesic submanifold of $M^{n}$, through the deformation by the gradient flow of Busemann function. The above theorem follows as a corollary of this more general structure result.

In 1978, Gromov [Gro78] used Toponogov Comparison Theorem to study the fundamental group directly, and proved: For $M^{n}$ with sectional curvature $\sec \geq 0$, $\mathfrak{n g}\left(\pi_{1}\left(M^{n}\right)\right) \leq C(n)=\frac{\operatorname{Vol}\left(\mathbb{S}^{n-1}\right)}{\operatorname{Vol}\left(\mathbb{D}^{n-1}\left(\frac{\pi}{6}\right)\right)}$, where $\mathbb{S}^{n-1}$ is the unit sphere in $\mathbb{R}^{n}, \mathbb{D}^{n-1}(r)$ is the geodesic ball with radius $r$ in $\mathbb{S}^{n-1}$.

For compact Riemannian manifolds with $R c \geq 0$, under the additional assumption of conjugate radius, Guofang Wei [Wei97] gave a uniform estimate on the generators of the fundamental group similar as in Gromov's result.

Back in 1968, Milnor [Mil68] proved that for complete Riemannian manifold $M^{n}$ with $R c \geq 0$, any finitely generated subgroup of $\pi_{1}\left(M^{n}\right)$, is polynomial growth of order $\leq n$. Furthermore, he posed the following conjecture:

Conjecture 0.3 (Milnor). For complete Riemannian manifold $\left(M^{n}, g\right)$ with $R c \geq 0$, $\pi_{1}\left(M^{n}\right)$ is finitely generated.

In the 1980s, Peter Li [Li86] used the heat kernel and Anderson [And90] used the property of covering maps, to prove Milnor conjecture for Euclidean (maximal) volume growth case respectively (moreover, they proved the fundamental group is finite in fact).

In 2000, Sormani [Sor00] used the excess estimate of Abresch and Gromoll [AG90] on the universal cover of manifolds, successfully proved Milnor conjecture for linear (minimal) volume growth case. 
Also in 2000, B. Wilking [Wil00] used Milnor's Theorem and the theory of discrete subgroup in Lie group to prove: If for any complete Riemannian manifold $\left(M^{n}, g\right)$ with $R c(g) \geq 0$ and $\pi_{1}\left(M^{n}\right)$ is an abelian group, we have $\pi_{1}\left(M^{n}\right)$ is finitely generated; then for any complete Riemannian manifold $\left(N^{n}, \tilde{g}\right)$ with $\operatorname{Rc}(\tilde{g}) \geq 0$, $\pi_{1}\left(N^{n}\right)$ is finitely generated.

Note the proof of the above Wilking's Theorem do not need to use Bochner formula in Riemannian geometry, only relies on the Bishop-Gromov Volume Comparison Theorem.

Recently, in 2011, V. Kapovitch and B. Wilking [KW11] proved the following local estimate of fundamental groups among other things :

Theorem 0.4 (Kapovitch and Wilking). For complete Riemannian manifold $\left(M^{n}, g\right)$ with $R c \geq 0$, any finitely generated subgroup $\Gamma$ of $\pi_{1}\left(M^{n}\right)$ satisfies $\mathfrak{n g}(\Gamma) \leq C(n)$.

Their proof was inspired by Fukaya and Yamaguchi's work [FY92], started by contradiction, used the equivariant pointed Gromov-Hausdorff convergence and reduced the problem to the study of Ricci limit space with group actions, then do the induction on the dimension of Ricci limit space. The main technical tools are Cheeger-Colding's theory of Ricci limit space and almost rigidity results. However this proof can not give the explicit estimate of the above $C(n)$.

One main purpose of this paper is to give an explicit uniform estimate of $\mathfrak{n g}(\Gamma)$ for finitely generated subgroup $\Gamma \subseteq \pi_{1}\left(M^{n}\right)$. More precisely, we prove the following theorem, which can be thought as the quantitative version of Theorem 0.4 .

Theorem 0.5. For complete Riemannian manifold $\left(M^{n}, g\right)$ with $R c \geq 0$, any finitely generated subgroup $\Gamma$ of $\pi_{1}\left(M^{n}\right)$ satisfies $n \mathrm{~g}(\Gamma) \leq n^{n^{20 n}}$.

Remark 0.6. The above upper bound $n^{n^{20 n}}$ is not sharp, and our method can not provide the sharp bound either. Also, comparing the concise proof of Theorem 0.4 in [KW11], our proof is sort of lengthy.

One advantage of our proof is self-contained. Basically, our argument only use Bishop-Gromov Volume Comparison Theorem and the Bochner formula for complete Riemannian manifolds with $R c \geq 0$. We try to reveal the relation between discrete isometry group actions and $R c \geq 0$ in an intrinsic way. Even the Compactness Theorem of Gromov-Hausdorff convergence is not used, let alone the theory of Ricci limit spaces, we only need the concept of $\epsilon$-Gromov-Hausdorff approximation.

There are three key ingredients of our proof. The first one tells us how to transfer from Gromov-Hausdorff approximation (geometry assumption) to almost orthonormal linear harmonic functions (analysis result). The second one is doing reverse argument, i.e. transferring analysis to geometry. And the third one studies the generating set of the covering groups through analysis and geometry. We will describe those three key ingredients in the rest of this section, and conclude the section with a sketchy description of our proof.

Firstly, we hope to reveal more explicit relation between group actions and the assumption $R c \geq 0$, through the concrete analysis on distance function, in the 
similar spirit of Gromov's proof about estimate of fundamental group's generators. This hope starts from the following classical global result of Cheeger and Gromoll [CG71] obtained in 1971:

Theorem 0.7 (Cheeger and Gromoll). If $\left(M^{n}, g\right)$ is a complete $n$-dim Riemannian manifold with $R c \geq 0$, and $M^{n}$ contains a line, then $M^{n}$ is isometric to $N^{n-1} \times \mathbb{R}$, where $N^{n-1}$ is a complete $(n-1)$-dim Riemannian manifold with $R c \geq 0$.

The proof of Theorem 0.7 used the harmonic function $\mathbf{b}$ globally defined on $M^{n}$, which is sort of the limit of the distance function of $M^{n}$ (more precisely, the Busemann function). In fact, the above manifold $N^{n-1}$ is a level set of $\mathbf{b}$ and the splitting lines $\mathbb{R}$ are the gradient flow lines of $\mathbf{b}$. One crucial technical point is to get the gradient estimate of the harmonic function $\mathbf{b}$, which is $|\nabla \mathbf{b}| \equiv 1$.

In 1975, Cheng and Yau [CY75] established the well-known local gradient estimate of harmonic functions on complete Riemannian manifolds with $R c \geq 0$, which enables us to obtain the gradient estimate of harmonic functions from the $C^{0}$-bound of harmonic functions locally. On such manifolds, in 1990, Abresch and Gromoll [AG90] obtained the excess estimate, which gives the local estimate of the sum of two local Busemann functions with respect to a segment (such sum is 0 for two Busemann functions with reverse directions in the proof of Theorem 0.7).

Then in the 1990s, based on the gradient estimate and the excess estimate, Colding initiated the study of local properties of distance function, through harmonic function locally defined on manifolds with Ricci lower bound, in a series paper [Col96a], [Col96b], [Col97]; while solving several important problems in the theory of Gromov-Hausdorff convergence, which was established by Gromov in the 1980s (for more details see [Gro99]). More precisely, among other things, Colding constructed the locally defined harmonic function based on the local Busemann function with respect to a segment, and proved such harmonic functions are 'almost linear' harmonic functions with bounded gradient, where the 'almost linear' is in average integral sense.

The first ingredient of our proof is the above existence of almost orthonormal linear harmonic functions, which was originally established by Colding in [Col96a], [Col96b], [Col97]. For our purpose, we need the explicit quantitative version, so we give all the details of the proof here. Although some calculation is sort of tedious, these explicit estimates possibly give an intrinsic expression of the transfer from geometry (Gromov-Hausdorff approximation) to analysis (existence of almost orthonormal linear harmonic functions).

In 1996, Cheeger and Colding [CC96] established the almost splitting theorem among other things, which transfers from analysis (existence of almost orthonormal linear harmonic functions) to geometry (Gromov-Hausdorff approximation). Very roughly, they proved that if there exist $k$ almost orthonormal linear harmonic functions on a geodesic ball $B_{r}(p) \subset M^{n}$ with $R c \geq 0$, then a smaller concentric geodesic ball is close to a ball of $\mathbb{R}^{k} \times \mathbf{X}_{k}$ in the sense of Gromov-Hausdorff distance.

This almost splitting theorem is the second ingredient of our proof. For the same reason as the above, we need the quantitative version. During the proof of the 
almost splitting theorem, one crucial thing is the existence of a suitable GromovHausdorff approximation under the suitable Hessian integral bound assumption. Because the original proof of this existence result in [CC96] is concise, one of our contribution is a different detailed proof by modifying some argument in [CN12], for more details see Section 5 of this paper.

It is a natural question whether the topology of two metric spaces are the same when they are very close in Gromov-Hausdorff distance sense. Generally, the answer is no, although there is an intrinsic Reifenberg type theorem when one of the spaces is $n$-dim Euclidean space and the other space is $n$-dim Riemannian manifolds (for details, see [CC97, Appendix]).

However, for a family of Riemannian manifolds converging to a metric space in Gromov-Hausdorff distance sense, using the equivariant Gromov-Hausdorff convergence theory developed by Fukaya and Yamaguchi in the 1980s (see [Fuk86], [FY92]), Kapovitch and Wilking [KW11] obtained the results, which relate the fundamental groups of those converging Riemannian manifolds, to the limit group, which acts on the limit space of the universal covers of those Riemannian manifolds.

The study of the fundamental groups can be put into a more general context, i.e. the covering group of a covering map between two Riemannian manifolds with $R c \geq 0$. The third ingredient of our proof is to characterize the change of the covering groups by the Gromov-Hausdorff distance between two metric spaces in quantitative form, where one metric space is a geodesic ball in Riemannian manifolds with $R c \geq 0$ and the other one is a ball in a product metric space $\mathbb{R}^{k} \times$ $\mathbf{X}_{k}$. Our squeeze lemma provides a bridge linking analysis with group actions and geometry. Very roughly, if there are $k$ almost orthonormal linear harmonic functions on a geodesic ball $B_{r}(p) \subset M^{n}$ with $R c \geq 0$ (analysis), then from the almost splitting theorem in the second ingredient, we know that $B_{c r}(p)$ is close to $B_{c r}(0, \hat{p}) \subset \mathbb{R}^{k} \times \mathbf{X}_{k}$ in Gromov-Hausdorff sense (geometry). Then the group actions on $B_{c r}(p)$ are almost generated by the group actions on $B_{c r}(\hat{p}) \subset \mathbf{X}_{k}$, for more details see Lemma 6.4

Now we describe our proof in a rough way. We start with a geodesic ball $B_{r}(p) \subset M^{n}$ with $R c \geq 0$, firstly we use the first ingredient tool to find one almost linear harmonic function, then apply Squeeze Lemma to shrink the group action to a group action on a ball $B_{c r}(0, \hat{p}) \subset \mathbf{X}_{n-1}$. Now we apply the first ingredient tool again to find two almost orthonormal linear harmonic functions on a smaller geodesic ball $B_{r_{1}}\left(p_{1}\right) \subset M^{n}$, and the group action on $B_{r}(p)$ can be 'controlled' or generated by the group action on $B_{r_{1}}\left(p_{1}\right)$. We repeat the above procedure by induction on the dimension of almost orthonormal linear harmonic functions. When the dimension is $n$, the group action is shown to be trivial, and we get our conclusion.

\section{PART I. G-H APPROXIMATION YIELDS A.O.L. HARMONIC FUNCTIONS}

In Part I of this paper, we will prove the following version result about analytic characterization of Gromov-Hausdorff approximation, which was implied in 
the argument of Cheeger and Colding in a series of papers, [Col96a], [Col96b], [Col97], [CC96] and [Che99].

Let us recall the definition of the pointed $\epsilon$-Gromov-Hausdorff approximation.

Definition 0.8. Let $\left(\mathbf{X}, d_{\mathbf{X}}, x_{0}\right)$ and $\left(\mathbf{Y}, d_{\mathbf{Y}}, y_{0}\right)$ be two pointed metric spaces. For $\epsilon>0$, a map $f:\left(\mathbf{X}, x_{0}\right) \rightarrow\left(\mathbf{Y}, y_{0}\right)$ is called a pointed $\epsilon$-Gromov-Hausdorff approximation if

$$
\begin{aligned}
& f\left(x_{0}\right)=y_{0}, \quad \mathbf{Y} \subset \mathbf{U}_{\epsilon}(f(\mathbf{X})), \\
& \left|d_{\mathbf{Y}}\left(f\left(x_{1}\right), f\left(x_{2}\right)\right)-d_{\mathbf{X}}\left(x_{1}, x_{2}\right)\right|<\epsilon, \quad \forall x_{1}, x_{2} \in \mathbf{X},
\end{aligned}
$$

where $\mathbf{U}_{\epsilon}(f(\mathbf{X})):=\{z \in \mathbf{Y}: d(z, f(\mathbf{X})) \leq \epsilon\}$. For simplicity, we also call that $f$ is an $\epsilon$-Gromov-Hausdorff approximation when the base points are fixed and clear.

In the rest of the paper, unless otherwise mentioned, we use $\mathbf{X}, \mathbf{Y}, \mathbf{X}_{k}$ to denote metric spaces. Also, we are only interested in geometry and analysis on $n$-dim manifolds with $n \geq 3$, so we will always assume the dimension of any manifolds $\mathbf{n} \geq \mathbf{3}$ in the rest of this paper.

For our application, we need the quantitative estimate, which relate the existence of the Gromov-Hausdorff approximation to the existence of almost orthonormal linear harmonic functions. Although many results in Part I are well-known to some experts in this field, we made the contribution to establish the suitable statement and the quantitative estimates. Also we elaborate the concise argument of Cheeger-Colding to present the proof of some known results in all the details for self-contained reason, and also hope to provide a backup reference for future study, besides the original works of Cheeger-Colding.

Theorem 0.9. For $B_{10 r}(q) \subset\left(M^{n}, g\right)$ with $\operatorname{Rc}(g) \geq 0$, any $0<\epsilon<1, \delta=$ $n^{-3400 n^{3}} \epsilon^{110 n}$ and integer $0 \leq k \leq n$, assume there is an ( $\left.\delta r\right)$-Gromov-Hausdorff approximation, $f: B_{10 r}(q) \rightarrow B_{10 r}(0, \hat{q}) \subset \mathbb{R}^{k} \times \mathbf{X}_{k}$, and $\operatorname{diam}\left(B_{r}(\hat{q})\right) \geq \frac{1}{4} r$ where $B_{r}(\hat{q}) \subset \mathbf{X}_{k}$. Then there are harmonic functions $\left\{\mathbf{b}_{i}\right\}_{i=1}^{k+1}$ defined on $B_{s}(p) \subset B_{10 r}(q)$, where $s=n^{-320 n^{3}} \epsilon^{10 n} r$, such that

$$
\sup _{\substack{B_{s}(p) \\ i=1, \cdots, k+1}}\left|\nabla \mathbf{b}_{i}\right| \leq 1+\epsilon \quad \text { and } \quad \sup _{t \leq s} f_{B_{t}(p)} \sum_{i, j=1}^{k+1}\left|\left\langle\nabla \mathbf{b}_{i}, \nabla \mathbf{b}_{j}\right\rangle-\delta_{i j}\right| \leq \epsilon .
$$

\section{Almost orthonormal local Busemann functions}

Cheng-Yau's gradient estimates was originally proved in [CY75], the following form include an explicit form of constant, which is needed in the later proof. The proof is the same as in [CY75], so we omit it.

Theorem 1.1 (Cheng-Yau's gradient estimates). Assume $\operatorname{Rc}\left(M^{n}\right) \geq 0, p \in M^{n}$, $B_{2 R}(p) \subset M^{n}, f: B_{2 R}(p) \rightarrow \mathbb{R}$ is a harmonic function and $f \in C\left(\overline{B_{2 R}(p)}\right)$, then

$$
\sup _{B_{R}(p)}|\nabla f| \leq \frac{60 n}{R} \sup _{B_{2 R}(p)}|f| \text {. }
$$


If $\Delta f=c_{0} \geq 0$ for $f \in C\left(\overline{B_{2 R}(p)}\right)$, then $\sup _{B_{R}(p)}|\nabla f| \leq \frac{200 n(R+1)}{R}\left[\sup _{B_{2 R}(p)}|f|+c_{0}\right]$.

Set $b^{+}(\cdot)=d(q, \cdot)-d(q, p): M^{n} \rightarrow \mathbb{R}$, the function $b^{+}$is called the local Busemann function with respect to the couple points $[p, q]$. And we define the positive part of a function $f$ as

$$
f_{+}(x)=\max \{f(x), 0\} .
$$

Lemma 1.2. Given $R>0, R c\left(M^{n}\right) \geq 0$ and $p, q \in M$ with $d(p, q)>2 R$, then

$$
\frac{1}{V\left(B_{R}(p)\right)} \int_{B_{R}(p)}\left|\Delta b^{+}\right| \leq \frac{3 n}{R}
$$

where $b^{+}(\cdot)=d(q, \cdot)-d(q, p): M^{n} \rightarrow \mathbb{R}$.

Proof: Note for any $x \in B_{R}(p)$,

$$
d(q, x) \geq d(q, p)-d(p, x)>2 R-R=R .
$$

By Laplace Comparison Theorem,

$$
\Delta b^{+}=\Delta d(q, \cdot) \leq \frac{n-1}{d(q, \cdot)}<\frac{n-1}{R}, \quad \text { on } B_{R}(p)
$$

hence the positive part of $\Delta b^{+}$satisfies

$$
\sup _{x \in B_{R}(p)}\left(\Delta b^{+}\right)_{+}(x) \leq \frac{n-1}{R} .
$$

By Bishop-Gromov Comparison Theorem,

$$
\frac{V\left(\partial B_{R}(p)\right)}{V\left(B_{R}(p)\right)} \leq \frac{n}{R}
$$

Now from (1.1), (1.2), we have

$$
\begin{aligned}
\int_{B_{R}(p)}\left|\Delta b^{+}\right| & =2 \int_{B_{R}(p)}\left(\Delta b^{+}\right)_{+}-\int_{B_{R}(p)} \Delta b^{+} \\
& \leq 2 V\left(B_{R}(p)\right) \max _{B_{R}(p)}\left(\Delta b^{+}\right)_{+}+\left|\int_{B_{R}(p)} \Delta b^{+}\right| \\
& \leq \frac{2(n-1)}{R} V\left(B_{R}(p)\right)+\left|\int_{\partial B_{R}(p)} \frac{\partial b^{+}}{\partial \vec{n}}\right| \\
& \leq \frac{2(n-1)}{R} V\left(B_{R}(p)\right)+V\left(\partial B_{R}(p)\right) \\
& \leq \frac{3 n}{R} V\left(B_{R}(p)\right) .
\end{aligned}
$$

Lemma 1.3. Suppose $\operatorname{Rc}\left(M^{n}\right) \geq 0, x \in M$, and the function $\mathbf{b}$ satisfies

$$
\left\{\begin{aligned}
\Delta \mathbf{b} & =0 & & \text { on } B_{4 R}(x) \\
\mathbf{b} & =b^{+} & & \text {on } \partial B_{4 R}(x),
\end{aligned}\right.
$$


where $b^{+}(\cdot)=d(q, \cdot)-d(q, p): M^{n} \rightarrow \mathbb{R}$. Then

$$
\begin{aligned}
f_{B_{4 R}(x)}\left|\nabla\left(\mathbf{b}-b^{+}\right)\right|^{2} & \leq 8 R \cdot f_{B_{4 R}(x)}\left|\Delta b^{+}\right|, \\
f_{B_{2 R}(x)}\left|\nabla^{2} \mathbf{b}\right|^{2} & \leq \frac{10^{8} \cdot n^{3}}{R^{2}}\left[1+\frac{d(p, x)}{R}\right]^{2} .
\end{aligned}
$$

Proof: From Maximum principle, one have $z_{1}, z_{2} \in \partial B_{4 R}(x)$ such that

$$
\mathbf{b}\left(z_{1}\right)=\sup _{z \in B_{4 R}(x)} \mathbf{b}(z), \quad \mathbf{b}\left(z_{2}\right)=\min _{z \in B_{4 R}(x)} \mathbf{b}(z) .
$$

Then for any $y \in B_{4 R}(x)$,

$$
\begin{aligned}
\mathbf{b}(y)-b^{+}(y) & \leq \mathbf{b}\left(z_{1}\right)-b^{+}(y)=b^{+}\left(z_{1}\right)-b^{+}(y)=d\left(q, z_{1}\right)-d(q, y) \\
& \leq d\left(z_{1}, y\right) \leq d\left(z_{1}, x\right)+d(x, y) \leq 8 R .
\end{aligned}
$$

Similarly, we have $\mathbf{b}(y)-b^{+}(y) \geq-d\left(z_{2}, y\right) \geq-8 R$. Hence

$$
\sup _{B_{4 R}(x)}\left|\mathbf{b}-b^{+}\right| \leq 8 R \text {. }
$$

From integration by parts, we get

$$
\begin{aligned}
\int_{B_{4 R}(x)}\left|\nabla\left(\mathbf{b}-b^{+}\right)\right|^{2} & =-\int_{B_{4 R}(x)}\left(\mathbf{b}-b^{+}\right) \Delta\left(\mathbf{b}-b^{+}\right)=\int_{B_{4 R}(x)}\left(\mathbf{b}-b^{+}\right) \Delta b^{+} \\
& \leq \sup _{B_{4 R}(x)}\left|\mathbf{b}-b^{+}\right| \int_{B_{4 R}(x)}\left|\Delta b^{+}\right| \leq 8 R \int_{B_{4 R}(x)}\left|\Delta b^{+}\right| .
\end{aligned}
$$

From $\Delta \mathbf{b}=0$ and Bochner's formula,

$$
\frac{1}{2} \Delta\left(|\nabla \mathbf{b}|^{2}\right)=\left|\nabla^{2} \mathbf{b}\right|^{2}+\langle\nabla \Delta \mathbf{b}, \nabla \mathbf{b}\rangle+R c(\nabla \mathbf{b}, \nabla \mathbf{b}) \geq\left|\nabla^{2} \mathbf{b}\right|^{2} .
$$

Let $\phi \in C^{\infty}\left(R_{+}\right)$be a nonnegative cut-off function such that

$$
\phi(s)= \begin{cases}1 & 0 \leq s \leq 2 R \\ 0 & s \geq 3 R\end{cases}
$$

and

$$
-\frac{2}{R} \leq \phi^{\prime} \leq 0, \quad\left|\phi^{\prime \prime}\right| \leq \frac{16}{R^{2}} .
$$

We use the notation $d_{p}(x)=d(p, x)$, similarly $d_{x}(\cdot)=d(x, \cdot)$. From Theorem 1.1 and Maximum principle,

$$
\sup _{B_{3 R}(x)}|\nabla \mathbf{b}| \leq \frac{60 n}{R} \sup _{B_{4 R}(x)}|\mathbf{b}| \leq \frac{60 n}{R} \sup _{\partial B_{4 R}(x)}\left|b^{+}\right| \leq 60 n \cdot\left[4+\frac{d_{p}(x)}{R}\right] .
$$

Then from (1.6) and (1.8),

$$
\begin{aligned}
\int_{B_{2 R}(x)}\left|\nabla^{2} \mathbf{b}\right|^{2} & \leq \int_{B_{3 R}(x)}\left|\nabla^{2} \mathbf{b}\right|^{2} \cdot\left(\phi \circ d_{x}\right) \leq \int_{B_{3 R}(x)} \frac{1}{2} \Delta\left(|\nabla \mathbf{b}|^{2}\right) \cdot\left(\phi \circ d_{x}\right) \\
(1.9) \quad & =\frac{1}{2} \int_{B_{3 R}(x)}|\nabla \mathbf{b}|^{2} \cdot \Delta\left(\phi \circ d_{x}\right) \leq 10^{6} n^{2}\left[1+\frac{d_{p}(x)}{R}\right]^{2} \int_{B_{3 R}(x)}\left|\Delta\left(\phi \circ d_{x}\right)\right| .
\end{aligned}
$$


On the other hand, from (1.7) and Laplace Comparison Theorem,

$$
\begin{aligned}
\int_{B_{3 R}(x)}\left|\Delta\left(\phi \circ d_{x}\right)\right| & =2 \int_{B_{3 R}(x)}\left(\Delta\left(-\phi \circ d_{x}\right)\right)_{+}-\int_{B_{3 R}(x)} \Delta\left(-\phi \circ d_{x}\right) \\
& \leq 2 V\left(B_{3 R}(x)\right) \max _{B_{3 R}(x)-B_{2 R}(x)}\left(\Delta\left(-\phi \circ d_{x}\right)\right)_{+}+\left|\int_{B_{3 R}(x)} \Delta\left(\phi \circ d_{x}\right)\right| \\
& \leq 2 V\left(B_{3 R}(x)\right) \max _{B_{3 R}(x)-B_{2 R}(x)}\left(-\phi^{\prime} \Delta d_{x}-\phi^{\prime \prime}\right)_{+}+\int_{\partial B_{3 R}(x)}\left|\frac{\partial\left(\phi \circ d_{x}\right)}{\partial \vec{n}}\right| \\
& \leq 2 V\left(B_{3 R}(x)\right) \max _{B_{3 R}(x)-B_{2 R}(x)}\left[\frac{2}{R} \cdot \frac{n-1}{d_{x}}+\left|\phi^{\prime \prime}\right|\right]+\sup _{\partial B_{3 R}(x)}\left|\phi^{\prime}\right| \cdot V\left(\partial B_{3 R}(x)\right) \\
& \leq 2 V\left(B_{3 R}(x)\right) \cdot \frac{20 n}{R^{2}}+\frac{2 n}{R^{2}} V\left(B_{3 R}(x)\right) \\
& \leq \frac{50 n}{R^{2}} V\left(B_{2 R}(x)\right) .
\end{aligned}
$$

The inequality (1.4) follows from (1.9) and (1.10).

Lemma 1.4. Let $0<\delta<\frac{1}{2}, R>0, D>1$ be given, suppose $R c\left(M^{n}\right) \geq 0, \tilde{p} \in M$, and $f, h \in L^{1}\left(B_{R}(\tilde{p})\right)$ with $f_{B_{R}(\tilde{p})}|f| \leq K, f_{B_{R}(\tilde{p})}|h| \leq \hat{K}$. Then there exists finite many disjoint balls $\left\{B_{\frac{1}{2} \delta R}\left(x_{j}\right)\right\}_{j \in \mathbf{J}}$ such that we have the following:

(I) For any $j \in \mathbf{J}, B_{\frac{1}{2} \delta R}\left(x_{j}\right) \subset B_{\left(1-\frac{3}{2} \delta\right) R}(\tilde{p})$ and $B_{2 \delta R}\left(x_{j}\right) \subset B_{R}(\tilde{p})$.

(II)

$$
\left\{\begin{aligned}
V\left(\cup_{i \in \mathbf{J}} B_{\delta R}\left(x_{i}\right)\right) & \geq\left[(1-2 \delta)^{n}-\frac{2 \cdot 10^{n}}{D}\right] V\left(B_{R}(\tilde{p})\right) \\
\sup _{i \in \mathbf{J}} f_{B_{2 \delta R}\left(x_{i}\right)}|f| & \leq D K \quad \text { and } \sup _{i \in \mathbf{J}} f_{B_{2 \delta R}\left(x_{i}\right)}|h| \leq D \hat{K} .
\end{aligned}\right.
$$

Proof: Choose a maximal set of disjoint balls $\left\{B_{\frac{1}{2} \delta R}\left(x_{i}\right)\right\}_{i \in \mathbf{S}}$ contained in $B_{\left(1-\frac{3}{2} \delta\right) R}(\tilde{p})$ and $B_{2 \delta R}\left(x_{i}\right) \subset B_{R}(\tilde{p})$. Assume $\tilde{q} \in \cap_{i=1}^{\lambda} B_{2 \delta R}\left(x_{i}\right)$, where $\{1, \cdots, \lambda\} \subset \mathbf{S}$, and note $B_{\frac{1}{2} \delta R}\left(x_{i}\right) \subset B_{\frac{5}{2} \delta R}(\tilde{q})$,

$$
\begin{aligned}
V\left(B_{\frac{5}{2} \delta R}(\tilde{q})\right) & \geq \sum_{i=1}^{\lambda} V\left(B_{\frac{1}{2} \delta R}\left(x_{i}\right)\right) \geq \sum_{i=1}^{\lambda}\left(\frac{\frac{1}{2} \delta R}{5 \delta R}\right)^{n} V\left(B_{5 \delta R}\left(x_{i}\right)\right) \\
& \geq \sum_{i=1}^{\lambda}\left[10^{-n} V\left(B_{\frac{5}{2} \delta R}(\tilde{q})\right)\right]=\lambda \cdot 10^{-n} V\left(B_{\frac{5}{2} \delta R}(\tilde{q})\right) .
\end{aligned}
$$

Hence $\lambda \leq 10^{n}$, and for all $\tilde{q} \in B_{R}(\tilde{p})$, there exists at most $10^{n}$-many $i$ with $\tilde{q} \in$ $B_{2 \delta R}\left(x_{i}\right)$.

Note $B_{(1-2 \delta) R}(\tilde{p}) \subset \cup_{i \in \mathbf{S}} B_{\delta R}\left(x_{i}\right)$ (otherwise, if $y \in B_{(1-2 \delta) R}(\tilde{p})-\cup_{i \in \mathbf{S}} B_{\delta R}\left(x_{i}\right)$, then $B_{\frac{1}{2} \delta R}(y) \cap B_{\frac{1}{2} \delta R}\left(x_{i}\right)=\emptyset$ for any $i \in \mathbf{S}$ and $B_{\frac{1}{2} \delta R}(y) \subset B_{\left(1-\frac{3}{2} \delta\right) R}(\tilde{p})$, contradicting the choice of $\left.\left\{B_{\frac{1}{2} \delta R}\left(x_{i}\right)\right\}_{i \in \mathbf{S}}\right)$.

Set

$$
\mathbf{I}=\left\{i \in \mathbf{S}\left|\int_{B_{2 \delta R}\left(x_{i}\right)}\right| f \mid \geq D K \cdot V\left(B_{2 \delta R}\left(x_{i}\right)\right) \text { or } \int_{B_{2 \delta R}\left(x_{i}\right)}|h| \geq D \hat{K} \cdot V\left(B_{2 \delta R}\left(x_{i}\right)\right)\right\} .
$$


Let $\mathbf{J}=\mathbf{S}-\mathbf{I}$, then

$$
\begin{aligned}
V\left(\cup_{j \in \mathbf{J}} B_{\delta R}\left(x_{j}\right)\right) & \geq V\left(\cup_{i \in \mathbf{S}} B_{\delta R}\left(x_{i}\right)\right)-V\left(\cup_{i \in \mathbf{I}} B_{\delta R}\left(x_{i}\right)\right) \\
& \geq V\left(B_{(1-2 \delta) R}(\tilde{p})\right)-V\left(\cup_{i \in \mathbf{I}} B_{\delta R}\left(x_{i}\right)\right) .
\end{aligned}
$$

Now we have

$$
\begin{aligned}
V\left(\cup_{i \in \mathbf{I}} B_{\delta R}\left(x_{i}\right)\right) & \leq \sum_{i \in \mathbf{I}} V\left(B_{2 \delta R}\left(x_{i}\right)\right) \leq \frac{1}{D K} \sum_{i \in \mathbf{I}} \int_{B_{2 \delta R}\left(x_{i}\right)}|f|+\frac{1}{D \hat{K}} \sum_{i \in \mathbf{I}} \int_{B_{2 \delta R}\left(x_{i}\right)}|h| \\
& \leq 10^{n} \cdot\left[\frac{1}{D K} \int_{B_{R}(\tilde{p})}|f|+\frac{1}{D \hat{K}} \int_{B_{R}(\tilde{p})}|h|\right] \leq \frac{2 \cdot 10^{n}}{D} V\left(B_{R}(\tilde{p})\right) .
\end{aligned}
$$

From (1.11) and (1.12), we have

$$
\begin{aligned}
& V\left(\cup_{j \in \mathbf{J}} B_{\delta R}\left(x_{j}\right)\right) \geq\left[(1-2 \delta)^{n}-\frac{2 \cdot 10^{n}}{D}\right] V\left(B_{R}(\tilde{p})\right) \\
& f_{B_{2 \delta R}\left(x_{i}\right)}|f| \leq D K \text { and } f_{B_{2 \delta R}\left(x_{i}\right)}|h| \leq D \hat{K}, \quad \forall i \in \mathbf{J} .
\end{aligned}
$$

Then $\left\{B_{\frac{1}{2} \delta R}\left(x_{j}\right)\right\}_{j \in \mathbf{J}}$ is our choice satisfying all properties required.

Lemma 1.5. Suppose $R c\left(M^{n}\right) \geq 0$, and $p, q \in M$ with $d(p, q)>2 r$. For all $\epsilon>0$, there exists finitely many balls $B_{\delta r}\left(x_{i}\right) \subset B_{r}(p), \forall i \in \mathbf{A}$, where $\mathbf{A}$ is a finite set, and harmonic functions $\mathbf{b}_{i}$ with $\delta=2^{-150 n} \epsilon^{18}$ such that

$$
\begin{array}{ll}
V\left(\bigcup_{i \in \mathbf{A}} B_{\delta r}\left(x_{i}\right)\right) \geq(1-\epsilon) V\left(B_{r}(p)\right), & \sum_{i \in \mathbf{A}} V\left(B_{\delta r}\left(x_{i}\right)\right) \leq 2^{2 n} V\left(B_{r}(p)\right), \\
f_{B_{2 \delta r}\left(x_{i}\right)}\left|\nabla\left(\mathbf{b}_{i}-b^{+}\right)\right| \leq \epsilon, & f_{B_{2 \delta r}\left(x_{i}\right)}\left|\nabla^{2} \mathbf{b}_{i}\right| \leq \frac{\epsilon}{\delta r},
\end{array}
$$

where $b^{+}(\cdot)=d(q, \cdot)-d(q, p): M^{n} \rightarrow \mathbb{R}$.

Proof: By scaling the metric $g$ to the new metric $r^{-2} g$, we only need to prove the conclusion for $r=1$.

From Lemma 1.2, we have $f_{B_{1}(p)}\left|\Delta b^{+}\right| \leq 3 n$. Combining this with Lemma 1.4, for $D>1$, and $\frac{1}{2}>\delta_{1}>0$ (to be determined later), we can choose finitely many balls $\left\{B_{\delta_{1}}\left(y_{j}\right)\right\}_{j \in \mathbf{J}}$ and $B_{2 \delta_{1}}\left(y_{j}\right) \subset B_{1}(p)$ such that

$$
\left\{\begin{array}{rlr}
B_{\frac{1}{2} \delta_{1}}\left(y_{j}\right) \cap B_{\frac{1}{2} \delta_{1}}\left(y_{i}\right) & =\emptyset, \quad i \neq j & \\
V\left(\cup_{j \in \mathbf{J}} B_{\delta_{1}}\left(y_{j}\right)\right) & \geq\left[\left(1-2 \delta_{1}\right)^{n}-\frac{2 \cdot 10^{n}}{D}\right] V\left(B_{1}(p)\right) \\
f_{B_{2 \delta_{1}}\left(y_{j}\right)}\left|\Delta b^{+}\right| & \leq D \cdot(3 n), \quad \forall j \in \mathbf{J} .
\end{array}\right.
$$

For each $j \in \mathbf{J}$, let $\mathbf{b}_{j}$ satisfy

$$
\left\{\begin{aligned}
\Delta \mathbf{b}_{j} & =0, & & \text { on } B_{2 \delta_{1}}\left(y_{j}\right) \\
\mathbf{b}_{j} & =b^{+}, & & \text {on } \partial B_{2 \delta_{1}}\left(y_{j}\right) .
\end{aligned}\right.
$$


Apply Lemma 1.3 to $B_{2 \delta_{1}}\left(y_{j}\right)$, we have

$$
\begin{aligned}
f_{B_{\delta_{1}}\left(y_{j}\right)}\left|\nabla\left(\mathbf{b}_{j}-b^{+}\right)\right|^{2} & \leq \frac{V\left(B_{2 \delta_{1}}\left(y_{j}\right)\right)}{V\left(B_{\delta_{1}}\left(y_{j}\right)\right)} f_{B_{2 \delta_{1}}\left(y_{j}\right)}\left|\nabla\left(\mathbf{b}_{j}-b^{+}\right)\right|^{2} \\
& \leq 2^{n+2} \delta_{1} \cdot f_{B_{2 \delta_{1}}\left(y_{j}\right)}\left|\Delta b^{+}\right| \leq D \cdot 2^{n+4} n \delta_{1}, \\
f_{B_{\delta_{1}}\left(y_{j}\right)}\left|\nabla^{2} \mathbf{b}_{j}\right|^{2} & \leq \frac{10^{8} n^{3}}{\left(\delta_{1} / 2\right)^{2}}\left[1+\frac{d_{p}\left(y_{j}\right)}{\delta_{1} / 2}\right]^{2} \leq \frac{10^{10} n^{3}}{\delta_{1}^{4}} .
\end{aligned}
$$

For any $j \in \mathbf{J}$, we can now apply Lemma 1.4 to the functions $\left|\nabla\left(\mathbf{b}_{j}-b^{+}\right)\right|^{2}$ and $\left|\nabla^{2} \mathbf{b}_{j}\right|^{2}$ on $B_{\delta_{1}}\left(y_{j}\right), j \in \mathbf{J}$. We get balls $\left\{B_{\delta_{2} \delta_{1}}\left(x_{i}^{j}\right)\right\}_{i, j}$, where $B_{2 \delta_{2} \delta_{1}}\left(x_{i}^{j}\right) \subset B_{\delta_{1}}\left(y_{j}\right)$ and let $\delta=\delta_{1} \delta_{2}$, such that

$$
\begin{aligned}
& f_{B_{2 \delta}\left(x_{i}^{j}\right)}\left|\nabla\left(\mathbf{b}_{j}-b^{+}\right)\right|^{2} \leq D \cdot f_{B_{\delta_{1}}\left(y_{j}\right)}\left|\nabla\left(\mathbf{b}_{j}-b^{+}\right)\right|^{2} \leq D^{2} \cdot 2^{n+4} n \delta_{1} \\
& f_{B_{2 \delta}\left(x_{i}^{j}\right)}\left|\nabla^{2} \mathbf{b}_{j}\right|^{2} \leq D \cdot f_{B_{\delta_{1}}\left(x_{j}\right)}\left|\nabla^{2} \mathbf{b}_{j}\right|^{2} \leq \frac{D \cdot 10^{10} n^{3}}{\delta_{1}^{4}}=\frac{D \cdot 10^{10} n^{3}\left(\delta_{2} \delta_{1}^{-1}\right)^{2}}{\delta^{2}}
\end{aligned}
$$

Furthermore, we set $\Omega_{j}=B_{\delta_{1}}\left(y_{j}\right)-\bigcup_{i} B_{\delta}\left(x_{i}^{j}\right)$, then

$$
\begin{aligned}
V\left(\Omega_{j}\right) & =V\left(B_{\delta_{1}}\left(y_{j}\right)\right)-V\left(\bigcup_{i} B_{\delta}\left(x_{i}^{j}\right)\right) \leq V\left(B_{\delta_{1}}\left(y_{j}\right)\right)-\left[\left(1-2 \delta_{2}\right)^{n}-\frac{2 \cdot 10^{n}}{D}\right] V\left(B_{\delta_{1}}\left(y_{j}\right)\right) \\
& =\left[1+\frac{2 \cdot 10^{n}}{D}-\left(1-2 \delta_{2}\right)^{n}\right] V\left(B_{\delta_{1}}\left(y_{j}\right)\right) .
\end{aligned}
$$

And we get

$$
\begin{aligned}
& \frac{V\left(\bigcup_{i, j} B_{\delta}\left(x_{i}^{j}\right)\right)}{V\left(B_{1}(p)\right)}=\frac{V\left(\bigcup_{j}\left[B_{\delta_{1}}\left(y_{j}\right)-\Omega_{j}\right]\right)}{V\left(B_{1}(p)\right)} \geq \frac{V\left(\bigcup_{j} B_{\delta_{1}}\left(y_{j}\right)\right)-\Sigma_{j} V\left(\Omega_{j}\right)}{V\left(B_{1}(p)\right)} \\
\geq & {\left[\left(1-2 \delta_{1}\right)^{n}-\frac{2 \cdot 10^{n}}{D}\right]-\left[1+\frac{2 \cdot 10^{n}}{D}-\left(1-2 \delta_{2}\right)^{n}\right] \sum_{j} \frac{V\left(B_{\delta_{1}}\left(y_{j}\right)\right)}{V\left(B_{1}(p)\right)} } \\
\geq & {\left[\left(1-2 \delta_{1}\right)^{n}-\frac{2 \cdot 10^{n}}{D}\right]-2^{n} \cdot\left[1+\frac{2 \cdot 10^{n}}{D}-\left(1-2 \delta_{2}\right)^{n}\right] \sum_{j} \frac{V\left(B_{\frac{1}{2} \delta_{1}}\left(y_{j}\right)\right)}{V\left(B_{1}(p)\right)} }
\end{aligned}
$$

If we choose $\delta_{2}=\delta_{1}^{2}, D=\delta_{1}^{-\frac{1}{3}} \cdot 10^{n}$. Using $\left(1-2 \delta_{1}\right)^{n} \geq 1-2 n \delta_{1}$, then for suitable $C(n)$, when $\delta_{1}=2^{-50 n} \epsilon^{6}, \delta=\delta_{2} \delta_{1}=2^{-150 n} \epsilon^{18}$, we get

$$
\begin{aligned}
D^{2} \cdot 2^{n+4} n \delta_{1} & \leq \epsilon^{2}, \\
D \cdot 10^{10} n^{3}\left(\delta_{2} \delta_{1}^{-1}\right)^{2} & \leq \epsilon^{2}, \\
\left(1-2 \delta_{1}\right)^{n}-\frac{2 \cdot 10^{n}}{D}-2^{n} \cdot\left[1+\frac{2 \cdot 10^{n}}{D}-\left(1-2 \delta_{2}\right)^{n}\right] & \geq 1-\epsilon .
\end{aligned}
$$


From (1.15), (1.16) and (1.17), we have

$$
f_{B_{2 \delta r}\left(x_{i}\right)}\left|\nabla\left(\mathbf{b}_{i}-b^{+}\right)\right|^{2} \leq \epsilon^{2}, \quad f_{B_{2 \delta r}\left(x_{i}\right)}\left|\nabla^{2} \mathbf{b}_{i}\right|^{2} \leq \frac{\epsilon^{2}}{\delta^{2} r^{2}} .
$$

Using Cauchy-Schwartz inequality, the conclusion follows.

Let $S M^{n}$ be the unit tangent bundle of $M^{n}$, if $\pi: S M^{n} \rightarrow M^{n}$ is the projection map, for any $\Omega \subset S M^{n}$, the Liouville measure of $\Omega$, denoted by $V(\Omega)$, is defined by $V(\Omega)=\mu(\pi(\Omega)) \cdot V\left(\mathbb{S}^{n-1}\right)$, where $V\left(\mathbb{S}^{n-1}\right)$ is the volume of the conical Euclidean $(n-1)$-sphere, and $\mu$ is the volume measure of $\left(M^{n}, g\right)$ determined by the metric $g$.

Definition 1.6. For $v \in S_{x} M^{n}$, let $\gamma_{v}(\cdot)$ be the geodesic starting from $x$ with $\gamma_{v}^{\prime}(0)=$ $v$, the geodesic flow $\mathfrak{g}^{t}(x, v):[0, \infty) \times S M^{n} \rightarrow S M^{n}$ is defined by

$$
\mathrm{g}^{t}(x, v)=\left(\gamma_{v}(t), \gamma_{v}^{\prime}(t)\right), \quad \forall t \geq 0
$$

Theorem 1.7 (Liouville's Theorem). For any region $D \subset S M^{n}$ we have $V\left(\mathrm{~g}^{t} D\right)=$ $V(D)$, where $\mathrm{g}^{t}: S M^{n} \rightarrow S M^{n}$ is the geodesic flow on $M^{n}$, and the measure on $D, \mathrm{~g}^{t} D$ is the Liouville measure.

Proof: [Arn89].

Lemma 1.8. Let $x \in M^{n}, l, r>0$, suppose $f \in C^{\infty}\left(B_{r+l}(x)\right)$, and $g$ is a Lipschitz function on $M^{n}$, then for any $0 \leq t \leq l$,

$$
f_{S B_{r}(x)}\left|\left(g \circ \gamma_{v}\right)^{\prime}(t)-\frac{g\left(\gamma_{v}(l)\right)-g\left(\gamma_{v}(0)\right)}{l}\right| \leq \frac{2}{V\left(B_{r}(x)\right)} \int_{B_{r+l}(x)}\left[l\left|\nabla^{2} f\right|+|\nabla(g-f)|\right] .
$$

Proof: Let $h(\tau)=f \circ \gamma_{v}(\tau)$, then for any $0 \leq t \leq l$

$$
\begin{aligned}
\left|h^{\prime}(t)-\frac{h(l)-h(0)}{l}\right| & =\left|\int_{0}^{t} h^{\prime \prime}(\tau) d \tau+h^{\prime}(0)-\frac{\int_{0}^{l}\left[\int_{0}^{s} h^{\prime \prime}(\tau) d \tau+h^{\prime}(0)\right] d s}{l}\right| \\
& \leq \int_{0}^{t}\left|h^{\prime \prime}\right| d \tau+\frac{\int_{0}^{l} \int_{0}^{s}\left|h^{\prime \prime}\right| d \tau d s}{l} \leq 2 \int_{0}^{l}\left|h^{\prime \prime}\right| d \tau
\end{aligned}
$$

Then

$$
\begin{aligned}
f_{S B_{r}(x)}\left|h^{\prime}(t)-\frac{h(l)-h(0)}{l}\right| & \leq \frac{2}{V\left(S B_{r}(x)\right)} \int_{S B_{r}(x)}\left(\int_{0}^{l}\left|\frac{\partial^{2}}{\partial \tau^{2}}\left(f \circ \gamma_{v}\right)\right| d \tau\right) \\
& =\frac{2}{V\left(B_{r}(x)\right) V\left(\mathbb{S}^{n-1}\right)} \int_{0}^{l}\left(\int_{S B_{r}(x)}\left|\frac{\partial^{2}}{\partial \tau^{2}}\left(f \circ \gamma_{v}\right)\right|\right) d \tau \\
& \leq \frac{2 l}{V\left(B_{r}(x)\right)} \int_{B_{r+l}(x)}\left|\nabla^{2} f\right|
\end{aligned}
$$


For any $0 \leq t \leq l$, applying Theorem 1.7 and (1.18),

$$
\begin{aligned}
& f_{S B_{r}(x)}\left|\left(g \circ \gamma_{v}\right)^{\prime}(t)-\frac{g\left(\gamma_{v}(l)\right)-g\left(\gamma_{v}(0)\right)}{l}\right| \leq f_{S B_{r}(x)}\left|\left(g \circ \gamma_{v}\right)^{\prime}(t)-\left(f \circ \gamma_{v}\right)^{\prime}(t)\right| \\
& \quad+f_{S B_{r}(x)}\left|h^{\prime}(t)-\frac{h(l)-h(0)}{l}\right|+f_{S B_{r}(x)} \frac{\int_{0}^{l}\left|\left((f-g) \circ \gamma_{v}\right)^{\prime}\right| d s}{l} \\
& \leq \frac{2 l}{V\left(B_{r}(x)\right)} \int_{B_{r+l}(x)}\left|\nabla^{2} f\right|+\frac{1}{V\left(S B_{r}(x)\right)} \int_{S B_{r+l}(x)}\left|\left(g \circ \gamma_{v}\right)^{\prime}(0)-\left(f \circ \gamma_{v}\right)^{\prime}(0)\right| \\
& \quad+\frac{1}{V\left(S B_{r}(x)\right)} \cdot \frac{1}{l} \int_{0}^{l}\left[\int_{S B_{r}(x)}\left|\left((f-g) \circ \gamma_{v}\right)^{\prime}(s)\right|\right] d s \\
& \leq \frac{2 l}{V\left(B_{r}(x)\right)} \int_{B_{r+l}(x)}\left|\nabla^{2} f\right|+\frac{2}{V\left(B_{r}(x)\right)} \int_{B_{r+l}(x)}|\nabla(g-f)| .
\end{aligned}
$$

Now we prove Colding's integral Toponogov theorem in quantitative form.

Theorem 1.9. Suppose $R c\left(M^{n}\right) \geq 0, p, q \in M$ with $d(p, q)>2 r$. For all $1>\epsilon>0$, there exists $\delta=2^{-240 n} \epsilon^{18}$ such that for all $0 \leq t \leq \delta$,

$$
f_{S B_{r}(p)}\left|\left(b^{+} \circ \gamma_{v}\right)^{\prime}(t r)-\frac{\left(b^{+} \circ \gamma_{v}\right)(\delta r)-\left(b^{+} \circ \gamma_{v}\right)(0)}{\delta r}\right| \leq \epsilon
$$

where $b^{+}(x)=d(x, q)-d(p, q)$.

Proof: From Lemma 1.5, for $\epsilon_{1}>0$ (to be determined later), we can find finitely many balls $B_{\delta r}\left(x_{i}\right) \subset B_{r}(p)$ with $\delta=2^{-150 n} \epsilon_{1}^{18}$ such that

$$
\begin{array}{ll}
V\left(\bigcup_{i \in \mathbf{A}} B_{\delta r}\left(x_{i}\right)\right) \geq\left(1-\epsilon_{1}\right) V\left(B_{r}(p)\right), & \\
f_{B_{2 \delta r}\left(x_{i}\right)}\left|\nabla\left(\mathbf{b}_{i}-b^{+}\right)\right| \leq \epsilon_{1}, & \sum_{i} V\left(B_{\delta r}\left(x_{i}\right)\right) \leq 2^{2 n} V\left(B_{r}(p)\right), \\
& f_{B_{2 \delta r}\left(x_{i}\right)}\left|\nabla^{2} \mathbf{b}_{i}\right| \leq \frac{\epsilon_{1}}{\delta r} .
\end{array}
$$

Let $h(v)=\left(b^{+} \circ \gamma_{v}\right)^{\prime}(t r)-\frac{\left(b^{+} \circ \gamma_{v}\right)(\delta r)-\left(b^{+} \circ \gamma_{v}\right)(0)}{\delta r}$, apply Lemma1.8, we get

$$
\begin{aligned}
& f_{S B_{\delta r}\left(x_{i}\right)}|h(v)| \leq \frac{2 \delta r}{V\left(B_{\delta r}\left(x_{i}\right)\right)} \int_{B_{2 \delta r}\left(x_{i}\right)}\left|\nabla^{2} \mathbf{b}_{i}\right|+\frac{2}{V\left(B_{\delta r}\left(x_{i}\right)\right)} \int_{B_{2 \delta r}\left(x_{i}\right)}\left|\nabla\left(\mathbf{b}_{i}-b^{+}\right)\right| \\
\leq & 2 \delta r \frac{V\left(B_{2 \delta r}\left(x_{i}\right)\right)}{V\left(B_{\delta r}\left(x_{i}\right)\right)} f_{B_{2 \delta r}\left(x_{i}\right)}\left|\nabla^{2} \mathbf{b}_{i}\right|+\frac{2 V\left(B_{2 \delta r}\left(x_{i}\right)\right)}{V\left(B_{\delta r}\left(x_{i}\right)\right)} f_{B_{2 \delta r}\left(x_{i}\right)}\left|\nabla\left(\mathbf{b}_{i}-b^{+}\right)\right| \\
\leq & 2^{n+2} \epsilon_{1} .
\end{aligned}
$$


Note $\left|\nabla b^{+}\right| \leq 1$, then we have

$$
\begin{aligned}
\int_{S B_{r}(p)}|h(v)| & \leq \int_{\bigcup_{i} S B_{\delta r}\left(x_{i}\right)}|h(v)|+2 V\left(S B_{r}(p)-\bigcup_{i} S B_{\delta r}\left(x_{i}\right)\right) \\
& \leq \sum_{i} \int_{S B_{\delta r}\left(x_{i}\right)}|h(v)|+2 V\left(\mathbb{S}^{n-1}\right) V\left(B_{r}(p)-\bigcup_{i} B_{\delta r}\left(x_{i}\right)\right) \\
& \leq 2^{n+2} \epsilon_{1} \sum_{i} V\left(S B_{\delta r}\left(x_{i}\right)\right)+2 V\left(\mathbb{S}^{n-1}\right) \cdot \epsilon_{1} V\left(B_{r}(p)\right) \\
& =2^{n+2} \epsilon_{1} \cdot V\left(\mathbb{S}^{n-1}\right) \sum_{i} V\left(B_{\delta r}\left(x_{i}\right)\right)+2 V\left(\mathbb{S}^{n-1}\right) \cdot \epsilon_{1} V\left(B_{r}(p)\right) \\
& \leq\left(2^{3 n+2}+2\right) \epsilon_{1} \cdot V\left(\mathbb{S}^{n-1}\right) V\left(B_{r}(p)\right)=\left(2^{3 n+2}+2\right) \epsilon_{1} \cdot V\left(S B_{r}(p)\right) .
\end{aligned}
$$

Let $\epsilon_{1}=2^{-5 n} \epsilon$, then we have the conclusion for $\delta=2^{-240 n} \epsilon^{18}$.

Proposition 1.10. Given $0<\epsilon<1,0 \leq k \leq n, B_{10 r}(q) \subset\left(M^{n}, g\right)$ with $R c(g) \geq 0$, any $\delta \leq n^{-1250 n} \cdot \epsilon^{100 n}\left(\frac{r_{1}}{r}\right)$ and $\frac{r_{1}}{r} \leq n^{-110 n} \epsilon^{10 n}$. Assume there is an $(\delta r)$-GromovHausdorff approximation $\Phi: B_{10 r}(0, \hat{q}) \rightarrow B_{10 r}(q)$, where $B_{10 r}(0, \hat{q}) \subset \mathbb{R}^{k} \times \mathbf{X}_{k}$, and there are $\hat{q}_{0}, \hat{q}_{1}^{+} \in B_{3 r}(\hat{q}) \subset \mathbf{X}_{k}$ satisfying $d\left(\hat{q}_{0}, \hat{q}_{1}^{+}\right)=r_{0} \in\left[\frac{1}{16} r, 2 r\right]$. Then we have

$$
f_{B_{r_{1}}\left(q_{1}\right)} \sum_{i, j=1}^{k+1}\left|\left\langle\nabla b_{i}^{+}, \nabla b_{j}^{+}\right\rangle-\delta_{i j}\right| \leq \epsilon,
$$

where $\left\{\mathbf{e}_{i}\right\}_{i=1}^{k}$ is the standard basis for $\mathbb{R}^{k}, q_{1}=\Phi\left(0, \hat{q}_{0}\right)$ and

$$
\begin{aligned}
b_{i}^{+}(\cdot) & =d\left(\cdot, p_{i}^{+}\right)-d\left(q_{1}, p_{i}^{+}\right), & p_{i}^{+}=\Phi\left(r_{0} \cdot \mathbf{e}_{i}, \hat{q}_{0}\right), & 1 \leq i \leq k \\
b_{k+1}^{+}(\cdot) & =d\left(\cdot, p_{k+1}^{+}\right)-d\left(q_{1}, p_{k+1}^{+}\right), & p_{k+1}^{+}=\Phi\left(0, \hat{q}_{1}^{+}\right) . &
\end{aligned}
$$

Proof: Step (1). Firstly we have

$$
d\left(\left(r_{0} \mathbf{e}_{i}, \hat{q}_{0}\right),(0, \hat{q})\right) \leq r_{0}^{2}+d\left(\hat{q}_{0}, \hat{q}\right)^{2} \leq(2 r)^{2}+(3 r)^{2}<(10 r)^{2},
$$

which implies $\left(r_{0} \mathbf{e}_{i}, \hat{q}_{0}\right) \in B_{10 r}(0, \hat{q})$, and $p_{i}^{+}$is well-defined. Also we can easily see $B_{r_{1}}\left(q_{1}\right) \subset B_{10 r}(q)$.

In this step we always assume that $x, y \in B_{r_{1}}\left(q_{1}\right)$. Because $\Phi$ is an $(\delta r)$-GromovHausdorff approximation, there exists $(\tilde{x}, \hat{x}),(\tilde{y}, \hat{y}) \in B_{10 r}(0, \hat{q})$ such that

$$
d(\Phi(\tilde{x}, \hat{x}), x)<\delta r \quad \text { and } \quad d(\Phi(\tilde{y}, \hat{y}), y)<\delta r .
$$

Let $d_{0}=d((\tilde{x}, \hat{x}),(\tilde{y}, \hat{y}))$, then

$$
\begin{aligned}
d_{0} & \leq\left|d(\Phi(\tilde{x}, \hat{x}), \Phi(\tilde{y}, \hat{y}))-d_{0}\right|+d(x, \Phi(\tilde{x}, \hat{x}))+d(y, \Phi(\tilde{y}, \hat{y}))+d(x, y) \\
& \leq 3 \delta r+d(x, y) .
\end{aligned}
$$

Assume $r_{1}=\zeta r$, we have

$$
\begin{aligned}
|\tilde{x}|^{2}+d\left(\hat{x}, \hat{q}_{0}\right)^{2} & \left.=d((\tilde{x}, \hat{x})),\left(0, \hat{q}_{0}\right)\right)^{2} \leq\left[d\left(\Phi(\tilde{x}, \hat{x}), \Phi\left(0, \hat{q}_{0}\right)\right)+\delta r\right]^{2} \\
& \leq\left[d\left(x, q_{1}\right)+2 \delta r\right]^{2} \leq\left(r_{1}+2 \delta r\right)^{2}=(\zeta+2 \delta)^{2} r^{2}
\end{aligned}
$$


which implies

$$
|\tilde{x}| \leq(\zeta+2 \delta) r \quad \text { and } \quad d\left(\hat{x}, \hat{q}_{0}\right) \leq(\zeta+2 \delta) r .
$$

Similarly we have

$$
|\tilde{y}| \leq(\zeta+2 \delta) r \quad \text { and } \quad d\left(\hat{y}, \hat{q}_{0}\right) \leq(\zeta+2 \delta) r .
$$

We define $\tilde{x}=\left(\tilde{x}_{1}, \cdots, \tilde{x}_{k}\right), \tilde{y}=\left(\tilde{y}_{1}, \cdots, \tilde{y}_{k}\right)$ and

$$
\begin{aligned}
& \mathcal{I}_{i}=\left|d\left((\tilde{x}, \hat{x}),\left(r_{0} \mathbf{e}_{i}, \hat{q}_{0}\right)\right)-d\left((\tilde{y}, \hat{y}),\left(r_{0} \mathbf{e}_{i}, \hat{q}_{0}\right)\right)\right| \\
& \mathcal{J}=\left|d\left((\tilde{x}, \hat{x}),\left(0, \hat{q}_{1}^{+}\right)\right)-d\left((\tilde{y}, \hat{y}),\left(0, \hat{q}_{1}^{+}\right)\right)\right| .
\end{aligned}
$$

From (1.21), (1.22) and (1.23),

$$
\begin{aligned}
\mathcal{I}_{i} & =\left|\sqrt{d\left(\tilde{x}, r_{0} \mathbf{e}_{i}\right)^{2}+d\left(\hat{x}, \hat{q}_{0}\right)^{2}}-\sqrt{d\left(\tilde{y}, r_{0} \mathbf{e}_{i}\right)^{2}+d\left(\hat{y}, \hat{q}_{0}\right)^{2}}\right| \\
& \leq \frac{\left|\sum_{j=1}^{k} \tilde{x}_{j}^{2}-\sum_{j=1}^{k} \tilde{y}_{j}^{2}\right|+2 r_{0}\left|\tilde{x}_{i}-\tilde{y}_{i}\right|+\left|d\left(\hat{x}, \hat{q}_{0}\right)^{2}-d\left(\hat{y}, \hat{q}_{0}\right)^{2}\right|}{\sqrt{d\left(\tilde{x}, r_{0} \mathbf{e}_{i}\right)^{2}+d\left(\hat{x}, \hat{q}_{0}\right)^{2}}+\sqrt{d\left(\tilde{y}, r_{0} \mathbf{e}_{i}\right)^{2}+d\left(\hat{y}, \hat{q}_{0}\right)^{2}}} \\
& \leq \frac{2(\zeta+2 \delta) r \cdot\left[\sum_{j=1}^{k}\left|\tilde{x}_{j}-\tilde{y}_{j}\right|+d(\hat{x}, \hat{y})\right]+2 r_{0} \cdot\left|\tilde{x}_{i}-\tilde{y}_{i}\right|}{\left|r_{0}-\tilde{x}_{i}\right|+\left|r_{0}-\tilde{y}_{i}\right|} \\
& \leq \frac{2(\zeta+2 \delta) r}{2 r_{0}-2(\zeta+2 \delta) r} \sqrt{k+1} d_{0}+\frac{2 r_{0}}{2 r_{0}-2(\zeta+2 \delta) r}\left|\tilde{x}_{i}-\tilde{y}_{i}\right| \\
& \leq \frac{8 n(\zeta+2 \delta)}{1-16(\zeta+2 \delta)}[d(x, y)+3 \delta r]+\frac{1}{1-16(\zeta+2 \delta)}\left|\tilde{x}_{i}-\tilde{y}_{i}\right|,
\end{aligned}
$$

in the last inequality above we used the assumption $r_{0} \geq \frac{1}{16} r$. Similarly, we have

$$
\begin{aligned}
\mathcal{J} & =\left|\sqrt{|\tilde{x}|^{2}+d\left(\hat{x}, \hat{q}_{1}^{+}\right)^{2}}-\sqrt{|\tilde{y}|^{2}+d\left(\hat{y}, \hat{q}_{1}^{+}\right)^{2}}\right| \\
& \leq \frac{\left|\sum_{j=1}^{k} \tilde{x}_{j}^{2}-\sum_{j=1}^{k} \tilde{y}_{j}^{2}\right|+\left|d\left(\hat{x}, \hat{q}_{1}^{+}\right)^{2}-d\left(\hat{y}, \hat{q}_{1}^{+}\right)^{2}\right|}{\sqrt{|\tilde{x}|^{2}+d\left(\hat{x}, \hat{q}_{1}^{+}\right)^{2}}+\sqrt{|\tilde{y}|^{2}+d\left(\hat{y}, \hat{q}_{1}^{+}\right)^{2}}} \\
& \leq \frac{2(\zeta+2 \delta) r \sum_{j=1}^{k}\left|\tilde{x}_{j}-\tilde{y}_{j}\right|+2\left(\zeta r+2 \delta r+r_{0}\right) \cdot d(\hat{x}, \hat{y})}{2 r_{0}-2(\zeta+2 \delta) r} \\
& \leq \frac{2 n(\zeta+2 \delta) r d_{0}}{2 r_{0}-2(\zeta+2 \delta) r}+\frac{1}{1-(\zeta+2 \delta) \frac{r}{r_{0}}} d(\hat{x}, \hat{y}) \\
& \leq \frac{8 n(\zeta+2 \delta)}{1-16(\zeta+2 \delta)}[d(x, y)+3 \delta r]+\frac{1}{1-16(\zeta+2 \delta)} d(\hat{x}, \hat{y}) .
\end{aligned}
$$


Then we get

$$
\begin{aligned}
\sqrt{\sum_{i=1}^{k} \mathcal{I}_{i}^{2}+\mathcal{J}^{2} \leq} & \frac{1}{1-16(\zeta+2 \delta)} d_{0}+\frac{8 n^{2}(\zeta+2 \delta)}{1-16(\zeta+2 \delta)}[d(x, y)+3 \delta r] \\
& +\frac{8 n(\zeta+2 \delta)}{1-16(\zeta+2 \delta)} \sqrt{d(x, y)+3 \delta r} \sqrt{d_{0}} \\
\leq & \left(\frac{1+16 n^{2}(\zeta+2 \delta)}{1-16(\zeta+2 \delta)}\right)[d(x, y)+3 \delta r]
\end{aligned}
$$

For $i \leq k$, we have

$$
\begin{aligned}
\left|b_{i}^{+}(x)-b_{i}^{+}(y)\right| & =\left|d\left(x, p_{i}^{+}\right)-d\left(y, p_{i}^{+}\right)\right| \\
& \leq\left|d\left(\Phi(\tilde{x}, \hat{x}), \Phi\left(r_{0} \cdot \mathbf{e}_{i}, \hat{q}_{0}\right)\right)-d\left((\tilde{y}, \hat{y}), \Phi\left(r_{0} \cdot \mathbf{e}_{i}, \hat{q}_{0}\right)\right)\right|+2 \delta r \\
& \leq 4 \delta r+\left|d\left((\tilde{x}, \hat{x}),\left(r_{0} \cdot \mathbf{e}_{i}, \hat{q}_{0}\right)\right)-d\left((\tilde{y}, \hat{y}),\left(r_{0} \cdot \mathbf{e}_{i}, \hat{q}_{0}\right)\right)\right| \\
& \leq 4 \delta r+\mathcal{I}_{i} \\
\left|b_{k+1}^{+}(x)-b_{k+1}^{+}(y)\right| & =\left|d\left(x, p_{k+1}^{+}\right)-d\left(y, p_{k+1}^{+}\right)\right| \\
& \leq\left|d\left(\Phi(\tilde{x}, \hat{x}), \Phi\left(0, \hat{q}_{1}^{+}\right)\right)-d\left(\Phi(\tilde{y}, \hat{y}), \Phi\left(0, \hat{q}_{1}^{+}\right)\right)\right|+2 \delta r \\
& \leq 4 \delta r+\left|d\left((\tilde{x}, \hat{x}),\left(0, \hat{q}_{1}^{+}\right)\right)-d\left((\tilde{y}, \hat{y}),\left(0, \hat{q}_{1}^{+}\right)\right)\right| \\
& \leq 4 \delta r+\mathcal{J} .
\end{aligned}
$$

Let $\Psi=\left(b_{1}^{+}, \cdots, b_{k}^{+}, b_{k+1}^{+}\right): M^{n} \rightarrow \mathbb{R}^{k+1}$, from (1.21), (1.25), (1.26) and (1.24),

$$
\begin{aligned}
& d(\Psi(x), \Psi(y))=\sqrt{\sum_{i=1}^{k+1}\left|b_{i}^{+}(x)-b_{i}^{+}(y)\right|^{2}} \\
\leq & \left\{\sum_{i=1}^{k}\left(16 \delta^{2} r^{2}+8 \delta r \cdot d_{0}+\mathcal{I}_{i}^{2}\right)+16 \delta^{2} r^{2}+8 \delta r \cdot d_{0}+\mathcal{J}^{2}\right\}^{\frac{1}{2}} \\
\leq & 4 n \delta r+4 n \delta \sqrt{r d_{0}}+\sqrt{\sum_{i=1}^{k} \mathcal{I}_{i}^{2}+\mathcal{J}^{2}} \\
\leq & 20 n \delta \cdot r+4 n \delta \cdot d(x, y)+\left(\frac{1+16 n^{2}(\zeta+2 \delta)}{1-16(\zeta+2 \delta)}\right)[d(x, y)+3 \delta r] .
\end{aligned}
$$

If we assume $d(x, y)=l r_{1}$, where $0<l<1$ is to be determined later, then

$$
\begin{aligned}
\frac{d(\Psi(x), \Psi(y))}{d(x, y)} & \leq 4 n \delta+\frac{1+16 n^{2}(\zeta+2 \delta)}{1-16(\zeta+2 \delta)}+\left(20 n+3 \cdot \frac{1+16 n^{2}(\zeta+2 \delta)}{1-16(\zeta+2 \delta)}\right) \frac{\delta \cdot r}{d(x, y)} \\
& \leq \frac{1+16 n^{2}(\zeta+2 \delta)}{1-16(\zeta+2 \delta)}+\left\{4 n \delta+\left(20 n+3 \cdot \frac{1+16 n^{2}(\zeta+2 \delta)}{1-16(\zeta+2 \delta)}\right) \frac{\delta}{l \zeta}\right\} .
\end{aligned}
$$


For $0<\epsilon_{1}<1$ (to be determined later), if the following holds:

$$
\zeta \leq \frac{\left(\frac{\epsilon_{1}}{4}\right)}{400 n^{2}} \quad \text { and } \quad \delta \leq \frac{\left(\frac{\epsilon_{1}}{4}\right) l}{800 n^{2}} \cdot \zeta
$$

from (1.27) and (1.28), we obtain

$$
\frac{d(\Psi(x), \Psi(y))}{d(x, y)} \leq 1+\frac{\epsilon_{1}}{4}, \quad \text { if } d(x, y)=l r_{1} \text { and } x, y \in B_{r_{1}}\left(q_{1}\right) \text {. }
$$

From (1.29), for all $v \in S B_{r_{1}}\left(q_{1}\right)$ except a zero-measure set, we have

$$
\frac{d\left(\Psi \circ \gamma_{v}\left(l r_{1}\right), \Psi \circ \gamma_{v}(0)\right)}{l r_{1}} \leq 1+\frac{\epsilon_{1}}{4}
$$

where $\gamma_{v}$ is the geodesic satisfying $\gamma_{v}^{\prime}(0)=v$.

Step (2). From Theorem 1.9, let

$$
l=2^{-240 n} \epsilon_{1}^{18}
$$

then we have

$$
\frac{1}{V\left(S B_{r_{1}}\left(q_{1}\right)\right)} \int_{S B_{r_{1}}\left(q_{1}\right)}\left|\left\langle\nabla b_{i}^{+}, v\right\rangle-f(v)\right| \leq \epsilon_{1}, \quad i=1, \cdots, k+1
$$

where $f(v):=\frac{\left(b_{i}^{+} \circ \gamma_{v}\right)\left(l r_{1}\right)-\left(b_{i}^{+} \circ \gamma_{v}\right)(0)}{l r_{1}}$ for $v \in S B_{r_{1}}\left(q_{1}\right)$.

For fixed $i$, and some $\theta \in\left(0, \frac{\pi}{2}\right)$ (to be determined later), set

$$
C_{\theta}=\left\{v \in S B_{r_{1}}\left(q_{1}\right) \mid \angle\left(v, \nabla b_{i}^{+}\right) \leq \theta\right\}=\left\{v \in S B_{r_{1}}\left(q_{1}\right) \mid\left\langle v, \nabla b_{i}^{+}\right\rangle \geq \cos \theta\right\},
$$

then for any $v \in C_{\theta}$,

$$
\left|v-\nabla b_{i}^{+}\right|^{2}=|v|^{2}+\left|\nabla b_{i}^{+}\right|^{2}-2\left\langle v, \nabla b_{i}^{+}\right\rangle \leq 2-2 \cos \theta .
$$

From (1.32),

$$
\begin{aligned}
& \frac{1}{V\left(S B_{r_{1}}\left(q_{1}\right)\right)} \int_{C_{\theta}}|| f(v)|-1| \\
\leq & \frac{1}{V\left(S B_{r_{1}}\left(q_{1}\right)\right)} \int_{C_{\theta}}|| f(v)\left|-\left\langle\nabla b_{i}^{+}, v\right\rangle\right|+\frac{1}{V\left(S B_{r_{1}}\left(q_{1}\right)\right)} \int_{C_{\theta}}\left|1-\left\langle\nabla b_{i}^{+}, v\right\rangle\right| \\
\leq & \epsilon_{1}+(1-\cos \theta) \cdot \frac{V\left(C_{\theta}\right)}{V\left(S B_{r_{1}}\left(q_{1}\right)\right)} \leq \epsilon_{1}+\theta \frac{V\left(C_{\theta}\right)}{V\left(S B_{r_{1}}\left(q_{1}\right)\right)} .
\end{aligned}
$$


Now from (1.32), (1.34) and (1.30), for $j \neq i$, we have

$$
\begin{aligned}
& \frac{1}{V\left(S B_{r_{1}}\left(q_{1}\right)\right)} \int_{C_{\theta}}\left|\left\langle\nabla b_{j}^{+}, v\right\rangle\right| \\
\leq & \frac{1}{V\left(S B_{r_{1}}\left(q_{1}\right)\right)} \int_{S B_{r_{1}}\left(q_{1}\right)}\left|\left\langle\nabla b_{j}^{+}, v\right\rangle-f(v)\right|+\frac{1}{V\left(S B_{r_{1}}\left(q_{1}\right)\right)} \int_{C_{\theta}}|f(v)| \\
\leq & \epsilon_{1}+\frac{V\left(C_{\theta}\right)^{\frac{1}{2}}}{V\left(S B_{r_{1}}\left(q_{1}\right)\right)}\left(\int_{C_{\theta}}\left|\frac{d\left(\Psi \circ \gamma_{v}\left(l r_{1}\right), \Psi \circ \gamma_{v}(0)\right)}{l r_{1}}\right|^{2}-|f(v)|^{2}\right)^{\frac{1}{2}} \\
\leq & \epsilon_{1}+\frac{V\left(C_{\theta}\right)^{\frac{1}{2}}}{V\left(S B_{r_{1}}\left(q_{1}\right)\right)}\left\{\epsilon_{1} V\left(C_{\theta}\right)^{\frac{1}{2}}+\left(\left.\int_{C_{\theta}}|1-| f(v)\right|^{2} \mid\right)^{\frac{1}{2}}\right\} \\
\leq & \epsilon_{1}+\frac{V\left(C_{\theta}\right)}{V\left(S B_{r_{1}}\left(q_{1}\right)\right)} \epsilon_{1}+\sqrt{2} \frac{V\left(C_{\theta}\right)^{\frac{1}{2}}}{V\left(S B_{r_{1}}\left(q_{1}\right)\right)} \cdot\left(\int_{C_{\theta}}|| f(v)|-1|\right)^{\frac{1}{2}} \\
\leq & 2 \epsilon_{1}+\sqrt{2}\left(\frac{V\left(C_{\theta}\right)}{V\left(S B_{r_{1}}\left(q_{1}\right)\right)}\right)^{\frac{1}{2}} \cdot\left(\epsilon_{1}+\theta \cdot \frac{V\left(C_{\theta}\right)}{V\left(S B_{r_{1}}\left(q_{1}\right)\right)}\right)^{\frac{1}{2}} \leq 4 \sqrt{\epsilon_{1}}+2 \sqrt{\theta} \frac{V\left(C_{\theta}\right)}{V\left(S B_{r_{1}}\left(q_{1}\right)\right)} .
\end{aligned}
$$

Note (1.33), then

$$
\begin{aligned}
\int_{C_{\theta}}\left|\left\langle\nabla b_{i}^{+}, \nabla b_{j}^{+}\right\rangle\right| & \leq \int_{C_{\theta}}\left|\left\langle\nabla b_{j}^{+}, v-\nabla b_{i}^{+}\right\rangle\right|+\int_{C_{\theta}}\left|\left\langle\nabla b_{j}^{+}, v\right\rangle\right| \\
& \leq \int_{C_{\theta}}\left|v-\nabla b_{i}^{+}\right|+\int_{C_{\theta}}\left|\left\langle\nabla b_{j}^{+}, v\right\rangle\right| \leq \theta \cdot V\left(C_{\theta}\right)+\int_{C_{\theta}}\left|\left\langle\nabla b_{j}^{+}, v\right\rangle\right| .
\end{aligned}
$$

Now note $\left\langle\nabla b_{i}^{+}, \nabla b_{j}^{+}\right\rangle$is constant on $T_{x} M^{n}$ for any fixed $x \in M^{n}$, we have

$$
\begin{aligned}
f_{B_{r_{1}}\left(q_{1}\right)}\left|\left\langle\nabla b_{i}^{+}, \nabla b_{j}^{+}\right\rangle\right| & =\frac{1}{V\left(C_{\theta}\right)} \int_{C_{\theta}}\left|\left\langle\nabla b_{i}^{+}, \nabla b_{j}^{+}\right\rangle\right| \\
& \leq \theta+\frac{V\left(S B_{r_{1}}\left(q_{1}\right)\right)}{V\left(C_{\theta}\right)} \cdot\left\{4 \sqrt{\epsilon_{1}}+2 \sqrt{\theta} \frac{V\left(C_{\theta}\right)}{V\left(S B_{r_{1}}\left(q_{1}\right)\right)}\right\} \\
& \leq 3 \sqrt{\theta}+4 \sqrt{\epsilon_{1}} \cdot \frac{V\left(S B_{r_{1}}\left(q_{1}\right)\right)}{V\left(C_{\theta}\right)} .
\end{aligned}
$$

Let $\epsilon_{1} \leq\left(\frac{V\left(C_{\theta}\right)}{4 V\left(S B_{r_{1}}\left(q_{1}\right)\right)}\right)^{2} \cdot \theta$ and $\theta=\frac{\epsilon^{2}}{16} \cdot \frac{1}{(n+1)^{4}}$, then $f_{B_{r_{1}}\left(q_{1}\right)}\left|\left\langle\nabla b_{i}^{+}, \nabla b_{j}^{+}\right\rangle\right| \leq 4 \sqrt{\theta}=$ $\frac{\epsilon}{(n+1)^{2}}$. So we let $\epsilon_{1}=2 n^{-50 n} \epsilon^{4 n+2}$, the above conclusion follows. Plug into (1.31) and (1.28), the corresponding $\zeta, \delta$ can be determined.

\section{EXISTENCE OF ALMOST ORTHONORMAL LINEAR (A.O.L.) HARMONIC FUNCTIONS}

Definition 2.1. For $q^{+}, q^{-}, p \in \mathbf{X}$, where $\mathbf{X}$ is a metric space, we say that $\left[q^{+}, q^{-}, p\right]$ is an AG-triple on $\mathrm{X}$ with the excess $s$ and the scale $t$ if

$$
\mathbf{E}(p)=s \quad \text { and } \quad \min \left\{d\left(p, q^{+}\right), d\left(p, q^{-}\right)\right\}=t,
$$

where $\mathbf{E}(\cdot)=d\left(\cdot, q^{+}\right)+d\left(\cdot, q^{-}\right)-d\left(q^{+}, q^{-}\right)$.

For $p \in M^{n}, r_{2} \geq r_{1} \geq 0$, we define $A_{r_{1}, r_{2}}(p)$ as the following:

$$
A_{r_{1}, r_{2}}(p)=\left\{x \in M^{n} \mid r_{1}<d(x, p)<r_{2}\right\} .
$$


We have the following Abresch-Gromoll lemma.

Lemma 2.2. On complete Riemannian manifold $\left(M^{n}, g\right)$ with $R c \geq 0$, assume that $\left[q^{+}, q^{-}, p\right]$ is an AG-triple with the excess $\leq \frac{1}{n} \frac{r^{2}}{R}$ and the scale $\geq R$, furthermore assume $R \geq 2^{2 n} r$, then $\sup _{B_{r}(p)} \mathbf{E} \leq 2^{6} \cdot\left(\frac{r}{R}\right)^{\frac{1}{n-1}} r$, where $\mathbf{E}(\cdot)=d\left(\cdot, q^{+}\right)+d\left(\cdot, q^{-}\right)-$ $d\left(q^{+}, q^{-}\right)$.

Proof: Define the function $\varphi(\rho)=\int_{\rho}^{2} \int_{t}^{2}\left(\frac{s}{t}\right)^{n-1} d s d t$, and $\epsilon$ solves

$$
\frac{\frac{R}{r}-3}{4(n-1)}=\frac{\varphi\left(\frac{\epsilon}{4}\right)}{\epsilon}, \quad 0<\epsilon \leq 4
$$

From $R \geq 2^{2 n} r>[3+4(n-1) \varphi(1)] r$, it is easy to see that $\epsilon$ exists and is unique.

By $R \geq 2^{2 n} r$ and (2.1), we have

$$
\varphi\left(\frac{\epsilon}{4}\right)=\epsilon \cdot \frac{\frac{R}{r}-3}{4(n-1)} \geq \frac{\epsilon}{8 n} \frac{R}{r} .
$$

Note for $\rho \leq 1$,

$$
\varphi(\rho)=\frac{1}{n}\left[\frac{1}{2} \rho^{2}+\frac{2^{n}}{n-2} \rho^{2-n}+4\left(-\frac{1}{n-2}-\frac{1}{2}\right)\right] \leq 2^{n} \rho^{2-n} .
$$

From (2.2) and (2.3), we obtain $2^{n}\left(\frac{\epsilon}{4}\right)^{2-n} \geq \frac{\epsilon}{8 n} \frac{R}{r}$, which implies $\epsilon \leq 2^{6} \cdot\left(\frac{r}{R}\right)^{\frac{1}{n-1}}$.

To prove the conclusion, we only need to prove that $\sup _{B_{r}(p)} \mathbf{E} \leq \epsilon r$. By contradiction, if $\sup _{B_{r}(p)} \mathbf{E}>\epsilon r$, then there exists $x_{0} \in B_{r}(p)$, such that

$$
\mathbf{E}\left(x_{0}\right)>\epsilon r \text {. }
$$

From $|\nabla \mathbf{E}| \leq 2$ and (2.1), we have

$$
\begin{aligned}
d\left(p, x_{0}\right) & \geq \frac{\mathbf{E}\left(x_{0}\right)-\mathbf{E}(p)}{2}>\frac{\epsilon r-\frac{1}{n} \frac{r^{2}}{R}}{2} \geq \frac{1}{2}\left(\epsilon r-\frac{2(n-1)}{\frac{R}{r}-3} \varphi(1) r\right) \\
& \geq \frac{1}{2}\left(\epsilon r-\frac{2(n-1)}{\frac{R}{r}-3} \varphi\left(\frac{\epsilon}{4}\right) r\right)=\frac{1}{2}\left(\epsilon r-\frac{\epsilon}{2} r\right)=\frac{\epsilon}{4} r,
\end{aligned}
$$

which implies $p \in A_{\frac{\epsilon}{4}, 2 r}\left(x_{0}\right)$.

Define the function $h: A_{\frac{\epsilon}{4} r, 2 r}\left(x_{0}\right) \rightarrow[0, \infty)$ by $\epsilon \leq 2^{6} \cdot\left(\frac{r}{R}\right)^{\frac{1}{n-1}}$, and it is easy to check $(\mathbf{E}-h) \geq 0$ on $\partial B_{2 r}\left(x_{0}\right)$.

For any $x \in \partial B_{\frac{\epsilon}{4} r}\left(x_{0}\right)$, using $|\nabla \mathbf{E}| \leq 2$, (2.4) and (2.1), we have

$$
\mathbf{E}(x)-h(x) \geq \mathbf{E}\left(x_{0}\right)-2 \cdot \frac{\epsilon}{4} r-\frac{2(n-1) r}{\frac{R}{r}-3} \varphi\left(\frac{\epsilon}{4}\right)>\frac{\epsilon}{2} r-\frac{2(n-1) r}{\frac{R}{r}-3} \varphi\left(\frac{\epsilon}{4}\right)=0,
$$

hence we obtain $\min _{x \in \partial A_{\frac{\epsilon}{4}, 2 r}\left(x_{0}\right)}(\mathbf{E}-h)(x) \geq 0$.

On the other hand, let $d=d\left(x, x_{0}\right)$, then $\varphi^{\prime \prime}\left(\frac{d}{r}\right)+\frac{n-1}{d} r \cdot \varphi^{\prime}\left(\frac{d}{r}\right)=1$. From $R c \geq 0$ and Laplace Comparison Theorem $\Delta d \leq \frac{n-1}{d}$, note $\varphi^{\prime} \leq 0$, for any $x \in A_{\frac{\epsilon}{4}, 2 r}\left(x_{0}\right)$, 
we have the following inequality in weak sense:

$$
\begin{aligned}
& \Delta h(x)=\frac{2(n-1)}{\frac{R}{r}-3} r\left[r^{-2} \varphi^{\prime \prime}+r^{-1} \varphi^{\prime} \Delta d\right] \geq \frac{2(n-1)}{R-3 r} \\
& \Delta \mathbf{E}(x) \leq \frac{n-1}{d\left(x, q^{+}\right)}+\frac{n-1}{d\left(x, q^{-}\right)} \leq \frac{2(n-1)}{R-3 r} .
\end{aligned}
$$

Then we get $\min _{x \in A_{\frac{\epsilon}{4}, 2 r}\left(x_{0}\right)} \Delta(\mathbf{E}-h)(x) \leq 0$.

From Weak Maximum Principle for weak superharmonic function (c.f. [GT01, Theorem 8.1]), note $p \in A_{\frac{\epsilon}{4} r, 2 r}\left(x_{0}\right)$, we have $(\mathbf{E}-h)(p) \geq \min _{\partial A_{\frac{\epsilon}{4}, 2 r}\left(x_{0}\right)}(\mathbf{E}-h) \geq 0$. And note $d\left(p, x_{0}\right)<r$, we obtain

$$
\mathbf{E}(p) \geq h(p)=\frac{2(n-1)}{\frac{R}{r}-3} \varphi\left(\frac{d\left(p, x_{0}\right)}{r}\right) \cdot r>\frac{2(n-1)}{\frac{R}{r}-3} \varphi(1) r>\frac{1}{n} \frac{r^{2}}{R},
$$

which is contradicting the assumption on the excess, the conclusion is proved.

The following lemma provides the existence of good cut-off function on manifolds with $R c \geq 0$, which will be used later.

Lemma 2.3. If $\operatorname{Rc}\left(M^{n}\right) \geq 0$ and $p \in M^{n}$, for any $\tau \in(0,1)$, there is a nonnegative smooth function $\phi: M^{n} \rightarrow[0,1]$

$$
\phi(x)= \begin{cases}1 & x \in B_{\tau r}(p) \\ 0 & x \notin B_{r}(p)\end{cases}
$$

satisfying $\sup _{x \in B_{r}(p)}|\Delta \phi(x)| \leq \frac{10^{15} n^{10}}{\tau^{2 n}(1-\tau)^{8}} r^{-2}$.

Proof: Scaling $\left(M^{n}, g\right)$ to $\left(M^{n}, r^{-2} g\right)$, then we only to construct $\phi(x)$ for $r=1$. We define $h_{1}(\rho)=\int_{\rho}^{1} \int_{t}^{1}\left(\frac{s}{t}\right)^{n-1} d s d t$ and

$$
W_{1}(x)=\frac{h_{1}(d(p, x))}{h_{1}(\tau)}, \quad h_{2}(\rho)=\int_{0}^{\rho} \int_{0}^{t}\left(\frac{s}{t}\right)^{n-1} d s d t
$$

It is easy to see

$$
\frac{1}{2 n}(1-\tau)^{2} \leq h_{1}(\tau) \leq \frac{\tau^{2-n}}{n(n-2)} .
$$

Using Laplace Comparison Theorem, it is straightforward to get $\Delta W_{1} \geq \frac{1}{h_{1}(\tau)}$. From the theory of elliptic equations of second order (c.f. [GT01]), we can define the function $W: \overline{B_{1}(p)}-B_{\tau}(p) \rightarrow \mathbb{R}$ satisfying

$$
\begin{cases}\Delta W=\frac{1}{h_{1}(\tau)} & \text { on } B_{1}(p)-\overline{B_{\tau}(p)} \\ W=1 & \text { on } \partial B_{\tau}(p) \\ W=0 & \text { on } \partial B_{1}(p)\end{cases}
$$

Apply the Maximum Principle to $W-W_{1}$ on $\overline{B_{1}(p)}-B_{\tau}(p)$, we get

$$
W \geq W_{1} \quad \text { on } \overline{B_{1}(p)}-B_{\tau}(p),
$$

which implies that $W \geq 0$. 
For any $x_{0} \in \partial B_{\frac{1}{2}(1+\tau)}(p)$, we define $W_{2}(x)=\frac{h_{2}\left(d\left(x_{0}, x\right)\right)}{h_{1}(\tau)}$. Note $B_{\frac{1}{2}(1-\tau)}\left(x_{0}\right) \subset$ $\left(\overline{B_{1}(p)}-B_{\tau}(p)\right)$, from Laplace Comparison Theorem again,

$$
\Delta\left(W-W_{2}\right) \geq 0 \quad \text { on } B_{\frac{1}{2}(1-\tau)}\left(x_{0}\right) .
$$

Then Maximum Principle yields

$$
W\left(x_{0}\right)-W_{2}\left(x_{0}\right) \leq \max _{\partial B_{\frac{1}{2}(1-\tau)}\left(x_{0}\right)}\left(W-W_{2}\right) .
$$

From the definition of $W$ and Maximum Principle, we know

$$
W \leq 1 \quad \text { on } \overline{B_{1}(p)}-B_{\tau}(p) .
$$

On $\partial B_{\frac{1}{2}(1-\tau)}\left(x_{0}\right)$, we have $W_{2}(x)=\frac{h_{2}\left(\frac{1}{2}(1-\tau)\right)}{h_{1}(\tau)}$. Also note $W_{2}\left(x_{0}\right)=0$, then we get

$$
W\left(x_{0}\right) \leq 1-\frac{h_{2}\left(\frac{1}{2}(1-\tau)\right)}{h_{1}(\tau)}, \quad \forall x_{0} \in \partial B_{\frac{1}{2}(1+\tau)}(p) .
$$

By $\Delta W(x) \geq 0$, apply Maximum Principle to $W$ on $\overline{B_{1}(p)}-B_{\frac{1}{2}(1+\tau)}(p)$, we have

$$
W \leq 1-\frac{h_{2}\left(\frac{1}{2}(1-\tau)\right)}{h_{1}(\tau)}, \quad \text { on } \overline{B_{1}(p)}-B_{\frac{1}{2}(1+\tau)}(p) .
$$

We define $\delta_{0}=\frac{(1-\tau)^{2} \tau}{16 n^{2}}$, then we can get

$$
\frac{h_{1}\left(\tau+\delta_{0}\right)}{h_{1}(\tau)}-\left(1-\frac{h_{2}\left(\frac{1}{2}(1-\tau)\right)}{h_{1}(\tau)}\right) \geq \frac{(1-\tau)^{2}}{32 n \cdot h_{1}(\tau)}
$$

We can find a smooth function $f:[0,1] \rightarrow[0,1]$ as the following:

$$
f(s)=\left\{\begin{array}{lr}
0 & 0 \leq s \leq 1-\frac{h_{2}\left(\frac{1}{2}(1-\tau)\right)}{h_{1}(\tau)} \\
1 & \frac{h_{1}\left(\tau+\delta_{0}\right)}{h_{1}(\tau)} \leq s \leq 1,
\end{array}\right.
$$

which satisfies

$$
\left|f^{\prime}(s)\right|+\left|f^{\prime \prime}(s)\right| \leq \frac{10^{4} n^{2}}{(1-\tau)^{4}}\left[h_{1}(\tau)^{2}+1\right] .
$$

Note when $\tau<d(x, p) \leq \tau+\delta_{0}$, from (2.6), we have $W(x) \geq W_{1}(x) \geq \frac{h_{1}\left(\tau+\delta_{0}\right)}{h_{1}(\tau)}$, then $f(W(x))=1$. And when $\frac{1}{2}(1+\tau) \leq d(x, p)<1$, from (2.7), we get $W(x) \leq$ $1-\frac{h_{2}\left(\frac{1}{2}(1-\tau)\right)}{h_{1}(\tau)}$, hence $f(W(x))=0$.

Now we can define smooth function $\phi$ as the following,

$$
\phi(x)= \begin{cases}1 & x \in B_{\tau}(p) \\ 0 & x \notin B_{1}(p) \\ f(W(x)) & x \in \overline{B_{1}(p)}-B_{\tau}(p)\end{cases}
$$


From $0 \leq W \leq 1$ and Theorem 1.1, for any $x$ satisfying $\tau+\delta_{0} \leq d(x, p) \leq$ $\frac{1}{2}(1+\tau)$,

$$
|\nabla W(x)| \leq \frac{200 n\left(\frac{1}{2} \delta_{0}+1\right)}{\frac{1}{2} \delta_{0}}\left[\sup _{B_{\delta_{0}}(x)}|W|+\frac{1}{h_{1}(\tau)}\right] \leq \frac{800 n\left(1+h_{1}(\tau)\right)}{\delta_{0} \cdot h_{1}(\tau)} .
$$

Then from (2.8) and (2.5), on $M^{n}$,

$$
|\Delta \phi| \leq\left|f^{\prime \prime}\right| \cdot|\nabla W|^{2}+\left|f^{\prime}\right| \cdot|\Delta W| \leq 10^{10} n^{4} \cdot \frac{\left(1+h_{1}(\tau)\right)^{4}}{\delta_{0}^{2} \cdot h_{1}(\tau)^{2}} \leq \frac{10^{15} n^{10}}{\tau^{2 n}(1-\tau)^{8}} .
$$

Lemma 2.4. On complete Riemannian manifold $M^{n}$ with $R c \geq 0$, for harmonic function $\mathbf{b}$ defined on $B_{r}(p)$ and any $\tau \in(0,1)$, we have

$$
f_{B_{\tau r}(p)}\left|\nabla^{2} \mathbf{b}\right| \leq \frac{10^{8} n^{5} r^{-1}}{\tau^{\frac{3 n}{2}}(1-\tau)^{4}} \cdot \sqrt{\sup _{B_{r}(p)}|\nabla \mathbf{b}|+1} \cdot \sqrt{f_{B_{r}(p)}|| \nabla \mathbf{b}|-1|} .
$$

Proof: From Bochner formula and $\Delta \mathbf{b}=0$, we have

$$
\frac{1}{2} \Delta|\nabla \mathbf{b}|^{2}=\left|\nabla^{2} \mathbf{b}\right|^{2}+R c(\nabla \mathbf{b}, \nabla \mathbf{b}) \geq\left|\nabla^{2} \mathbf{b}\right|^{2}
$$

From Lemma 2.3, one can choose a nonnegative cut-off function $\phi$ such that

$$
\phi(x)= \begin{cases}1 & x \in B_{\tau r}(p) \\ 0 & x \notin B_{r}(p)\end{cases}
$$

satisfying $\sup _{x \in B_{r}(p)}|\Delta \phi(x)| \leq \frac{10^{15} n^{10}}{\tau^{2 n}(1-\tau)^{8}} r^{-2}$. Now we have

$$
\begin{aligned}
\int_{B_{\tau r}(p)}\left|\nabla^{2} \mathbf{b}\right|^{2} & \leq \int_{B_{r}(p)}\left|\nabla^{2} \mathbf{b}\right|^{2} \cdot \phi \leq \frac{1}{2} \int_{B_{r}(p)} \Delta\left(|\nabla \mathbf{b}|^{2}\right) \cdot \phi \\
& =\frac{1}{2} \int_{B_{r}(p)}|\nabla \mathbf{b}|^{2} \cdot \Delta \phi=\frac{1}{2} \int_{B_{r}(p)}\left(|\nabla \mathbf{b}|^{2}-1\right) \cdot \Delta \phi \\
& \leq\left.\frac{1}{2} \sup _{B_{r}(p)}|\Delta \phi| \cdot \int_{B_{r}(p)}|| \nabla \mathbf{b}\right|^{2}-1 \mid \\
& \leq \frac{\tau^{-n}}{2} \sup _{B_{r}(p)}|\Delta \phi| \cdot\left(\sup _{B_{r}(p)}|\nabla \mathbf{b}|+1\right) V\left(B_{\tau r}(p)\right) f_{B_{r}(p)}|| \nabla \mathbf{b}|-1| .
\end{aligned}
$$

From Cauchy-Schwartz inequality and the above inequality, we have

$$
f_{B_{\tau r}(p)}\left|\nabla^{2} \mathbf{b}\right| \leq\left(f_{B_{\tau r}(p)}\left|\nabla^{2} \mathbf{b}\right|^{2}\right)^{\frac{1}{2}} \leq \frac{10^{8} n^{5} r^{-1}}{\tau^{\frac{3 n}{2}}(1-\tau)^{4}} \sqrt{\left(\sup _{B_{r}(p)}|\nabla \mathbf{b}|+1\right) f_{B_{r}(p)}|| \nabla \mathbf{b}|-1| .}
$$

Theorem 2.5 (Li-Yau). Let $\left(M^{n}, g\right)$ be a complete Riemannian manifold with $R c \geq$ 0 , then the heat kernel $H(x, y, t)$ satisfies

$$
H(x, y, t) \leq(100 n)^{2 n+2} \frac{1}{V\left(B_{\sqrt{t}}(y)\right)} \exp \left\{-\frac{d^{2}(x, y)}{100 t}\right\} .
$$


Proof: By choosing suitable $\epsilon$ in [LY86, Corollary 3.1], the conclusion follows.

Now we have the following existence result of almost linear harmonic function b with respect to the local Busemann function $b^{+}$.

Lemma 2.6. On complete Riemannian manifold $M^{n}$ with $R c \geq 0$, assume that $\left[q^{+}, q^{-}, p\right]$ is an AG-triple with the excess $\leq \frac{4}{n} \frac{r^{2}}{R}$ and the scale $\geq R$, also assume $R \geq 2^{2 n+1} r$. Then there exists harmonic function $\mathbf{b}$ defined on $B_{2 r}(p)$ such that

$$
\sup _{B_{r}(p)}|\nabla \mathbf{b}| \leq 1+2^{51 n^{2}}\left(\frac{r}{R}\right)^{\frac{1}{4(n-1)}} \quad \text { and } \quad f_{B_{r}(p)}\left|\nabla\left(\mathbf{b}-b^{+}\right)\right| \leq 2^{4 n}\left(\frac{r}{R}\right)^{\frac{1}{2(n-1)}}
$$

where $b^{+}(x)=d\left(x, q^{+}\right)-d\left(p, q^{+}\right)$.

Remark 2.7. The bound of $|\nabla \mathbf{b}|$ was only a uniform bound $C(n)$ in [CC96], it was observed in [CN15] that $|\nabla \mathbf{b}|$ can have the improved bound $1+\epsilon$. The argument to get $|\nabla \mathbf{b}| \leq 1+2^{51 n^{2}}\left(\frac{r}{R}\right)^{\frac{1}{4(n-1)}}$, comes from (3.42) - (3.45) of [CN15] , which was suggested to us by A. Naber.

Proof: Step (1). We define the function $\mathbf{b}$ by

$$
\begin{cases}\Delta \mathbf{b}=0 & \text { on } B_{2 r}(p) \\ \mathbf{b}=b^{+} & \text {on } \partial B_{2 r}(p) .\end{cases}
$$

Define $\psi(\rho)=\int_{\rho}^{4} \int_{t}^{4}\left(\frac{s}{t}\right)^{n-1} d s d t$, choose $q \in B_{6 r}(p)-\overline{B_{4 r}(p)}$, and define the function $\tilde{h}(x): A_{2 r, 8 r}(q) \rightarrow[0, \infty)$ by $\tilde{h}(x)=\frac{2(n-1) r}{\frac{R}{2 r}-7} \psi\left(\frac{d(x, q)}{2 r}\right)$. From Laplace Comparison Theorem, it is straightforward to get

$$
\min _{x \in A_{2 r, 8 r}(q)} \Delta\left(\tilde{h}-b^{+}\right)(x) \geq 0 .
$$

Note $B_{2 r}(p) \subset A_{2 r, 8 r}(q)$, hence $\min _{x \in B_{2 r}(p)} \Delta\left(\mathbf{b}-b^{+}+\tilde{h}\right)(x) \geq 0$.

From Maximum principle, for any $x \in B_{2 r}(p)$, we get

$$
\begin{aligned}
\left(\mathbf{b}-b^{+}\right)(x) & \leq\left(\mathbf{b}-b^{+}+\tilde{h}\right)(x) \leq \max _{\partial B_{2 r}(p)}\left(\mathbf{b}-b^{+}+\tilde{h}\right)=\max _{\partial B_{2 r}(p)} \tilde{h} \leq \frac{n-1}{\frac{R}{2 r}-7} \psi(1) \cdot 2 r \\
& \leq 4^{n} \cdot \frac{2 r}{R} \cdot 2 r \leq 2^{2 n+2} \frac{r^{2}}{R} .
\end{aligned}
$$

Let $b^{-}(x)=d\left(x, q^{-}\right)-d\left(p, q^{-}\right)$, then note $\mathbf{E}(x)=b^{+}(x)+b^{-}(x)+\mathbf{E}(p)$, then from $R \geq 2^{2 n+1} r$ and Lemma 2.2. $\sup _{B_{2 r}(p)} \mathbf{E}(x) \leq 2^{8}\left(\frac{r}{R}\right)^{\frac{1}{n-1}} r$, and we have

$$
\mathbf{b}-b^{+}=\mathbf{b}+b^{-}-\mathbf{E}+\mathbf{E}(p) \geq \mathbf{b}+b^{-}-2^{8}\left(\frac{r}{R}\right)^{\frac{1}{n-1}} r, \quad \text { on } B_{2 r}(p) .
$$

From Laplace Comparison Theorem again,

$$
\Delta\left(\mathbf{b}+b^{-}-2^{8}\left(\frac{r}{R}\right)^{\frac{1}{n-1}} r-\tilde{h}\right)=\Delta\left(b^{-}-\tilde{h}\right) \leq \frac{n-1}{d\left(x, q^{-}\right)}-\frac{n-1}{R-14 r} \leq 0, \quad \text { on } B_{2 r}(p) .
$$


By Maximum Principle, note $\mathbf{E}(x) \geq 0$ and the assumption on the excess, we get

$$
\begin{aligned}
& \min _{x \in B_{2 r}(p)}\left(\mathbf{b}+b^{-}-2^{8}\left(\frac{r}{R}\right)^{\frac{1}{n-1}} r-\tilde{h}\right)(x) \\
\geq & \min _{\partial B_{2 r}(p)}\left(\mathbf{b}+b^{-}-2^{8}\left(\frac{r}{R}\right)^{\frac{1}{n-1}} r-\tilde{h}\right) \\
\geq & \min _{\partial B_{2 r}(p)}\left(b^{+}+b^{-}\right)-2^{8}\left(\frac{r}{R}\right)^{\frac{1}{n-1}} r-\frac{n-1}{\frac{R}{2 r}-7} \psi(1) \cdot 2 r \\
= & \min _{\partial B_{2 r}(p)}(\mathbf{E}(x)-\mathbf{E}(p))-2^{8}\left(\frac{r}{R}\right)^{\frac{1}{n-1}} r-\frac{n-1}{\frac{R}{2 r}-7} \psi(1) \cdot 2 r \\
\geq & -\frac{1}{n} \frac{4 r^{2}}{R}-2^{8}\left(\frac{r}{R}\right)^{\frac{1}{n-1}} r-\frac{n-1}{\frac{R}{2 r}-7} \psi(1) \cdot 2 r \\
\geq & -2^{3 n}\left(\frac{r}{R}\right)^{\frac{1}{n-1}} r .
\end{aligned}
$$

From (2.10) and $\tilde{h} \geq 0$, we obtain

$$
\min _{x \in B_{2 r}(p)}\left(\mathbf{b}-b^{+}\right) \geq \mathbf{b}+b^{-}-2^{8}\left(\frac{r}{R}\right)^{\frac{1}{n-1}} r-\tilde{h} \geq-2^{3 n}\left(\frac{r}{R}\right)^{\frac{1}{n-1}} r .
$$

By (2.9) and (2.11), it yields

$$
\sup _{B_{2 r}(p)}\left|\mathbf{b}-b^{+}\right| \leq 2^{3 n}\left(\frac{r}{R}\right)^{\frac{1}{n-1}} r
$$

which implies

$$
\sup _{B_{2 r}(p)}|\mathbf{b}| \leq 2^{3 n}\left(\frac{r}{R}\right)^{\frac{1}{n-1}} r+2 r
$$

From Theorem 1.1 and (2.13), note $\frac{R}{2 r} \geq 1$, we get

$$
\sup _{B_{\frac{15}{8} r}(p)}|\nabla \mathbf{b}| \leq \frac{60 n}{\frac{1}{16} r} \sup _{B_{2 r}(p)}|\mathbf{b}| \leq 2^{4 n+12}
$$

Note $d\left(p, q^{+}\right) \geq R>4 r$, then from Lemma 1.2, $f_{B_{2 r}(p)}\left|\Delta b^{+}\right| \leq \frac{3 n}{2 r}$. Now do integration by parts, from (2.12), we get

$$
\begin{aligned}
f_{B_{2 r}(p)}\left|\nabla\left(\mathbf{b}-b^{+}\right)\right|^{2} & =f_{B_{2 r}(p)} \Delta b^{+} \cdot\left(\mathbf{b}-b^{+}\right) \leq \sup _{B_{2 r}(p)}\left|\mathbf{b}-b^{+}\right| \cdot f_{B_{2 r}(p)}\left|\Delta b^{+}\right| \\
& \leq 2^{3 n}\left(\frac{r}{R}\right)^{\frac{1}{n-1}} \cdot(3 n) \leq 2^{7 n}\left(\frac{r}{R}\right)^{\frac{1}{n-1}} .
\end{aligned}
$$

From (2.15) and the Bishop-Gromov Comparison Theorem, we have

$$
f_{B_{r}(p)}\left|\nabla\left(\mathbf{b}-b^{+}\right)\right| \leq\left(f_{B_{r}(p)}\left|\nabla\left(\mathbf{b}-b^{+}\right)\right|^{2}\right)^{\frac{1}{2}} \leq\left[2^{n} f_{B_{2 r}(p)}\left|\nabla\left(\mathbf{b}-b^{+}\right)\right|^{2}\right]^{\frac{1}{2}} \leq 2^{4 n}\left(\frac{r}{R}\right)^{\frac{1}{2(n-1)}} \text {. }
$$


Step (2). Also 2.15) implies the following

$$
\begin{aligned}
f_{B_{\frac{7}{4} r}(p)}|| \nabla \mathbf{b}|-1| & \leq f_{B_{\frac{7}{4} r}(p)}\left|\nabla \mathbf{b}-\nabla b^{+}\right| \leq\left(\frac{8}{7}\right)^{n} f_{B_{2 r}(p)}\left|\nabla \mathbf{b}-\nabla b^{+}\right| \\
& \leq\left(\frac{8}{7}\right)^{n}\left(f_{B_{2 r}(p)}\left|\nabla\left(\mathbf{b}-b^{+}\right)\right|^{2}\right)^{\frac{1}{2}} \leq 2^{7 n}\left(\frac{r}{R}\right)^{\frac{1}{2(n-1)}} \\
f_{B_{\frac{15}{8} r}(p)}|| \nabla \mathbf{b}|-1| & \leq 2^{7 n}\left(\frac{r}{R}\right)^{\frac{1}{2(n-1)}} .
\end{aligned}
$$

From (2.14), (2.17) and apply Lemma 2.4 for $\tau=\frac{14}{15}$ there, we have

$$
\begin{aligned}
f_{B_{\frac{7}{4}}(p)}\left|\nabla^{2} \mathbf{b}\right| & \leq 2^{7 n} 10^{8} n^{5} r^{-1} \sqrt{\sup _{B_{\frac{15}{8}}(p)}(|\nabla \mathbf{b}|+1) \cdot f_{B_{\frac{15}{8} r}(p)}|| \nabla \mathbf{b}|-1|} \\
& \leq 2^{27 n} r^{-1} \cdot \sqrt{2^{20 n} \cdot\left(\frac{r}{R}\right)^{\frac{1}{2(n-1)}}} \leq 2^{37 n} r^{-1}\left(\frac{r}{R}\right)^{\frac{1}{4(n-1)}} .
\end{aligned}
$$

From the Bochner formula and $\Delta \mathbf{b}=0$, using $R c \geq 0$, we have $\Delta|\nabla \mathbf{b}|^{2} \geq 2\left|\nabla^{2} \mathbf{b}\right|^{2}$, combining

$$
\left|\nabla^{2} \mathbf{b}\right| \geq|\nabla| \nabla \mathbf{b}||
$$

we get

$$
\Delta|\nabla \mathbf{b}| \geq \frac{\left|\nabla^{2} \mathbf{b}\right|^{2}-|\nabla| \nabla \mathbf{b}||^{2}}{|\nabla \mathbf{b}|} \geq 0 .
$$

Let $\phi \in C^{\infty}\left(M^{n}\right)$ be a nonnegative cut-off function such that

$$
\phi(x)= \begin{cases}1 & x \in B_{\frac{3}{2} r}(p) \\ 0 & x \notin B_{\frac{7}{4} r}(p),\end{cases}
$$

and

$$
-\frac{8}{r} \leq \phi^{\prime} \leq 0, \quad\left|\phi^{\prime \prime}\right| \leq \frac{64}{r^{2}} .
$$

Now for any $y \in B_{r}(p)$, from (2.19), (2.20) and (2.21), we have

$$
\begin{aligned}
& \frac{d}{d t} \int_{B_{2 r}(p)}(|\nabla \mathbf{b}|-1) \phi \cdot H(x, y, t) d x=\int_{B_{2 r}(p)}(|\nabla \mathbf{b}|-1) \phi \cdot \Delta_{x} H(x, y, t) d x \\
= & \int_{B_{2 r}(p)}\{\phi \cdot \Delta(|\nabla \mathbf{b}|-1)+(|\nabla \mathbf{b}|-1) \cdot \Delta \phi+2 \nabla|\nabla \mathbf{b}| \cdot \nabla \phi\} H(x, y, t) d x \\
(2.22) \geq & -\int_{A_{\frac{3 r}{2}, \frac{7}{4} r}(p)}\left[|| \nabla \mathbf{b}|-1| \cdot \frac{64}{r^{2}}+2\left|\nabla^{2} \mathbf{b}\right| \cdot \frac{8}{r}\right] H(x, y, t) d x .
\end{aligned}
$$


From Theorem 2.5, for $x \in A_{\frac{3 r}{2}, \frac{7}{4} r}(p), y \in B_{r}(p)$, we have

$$
\begin{aligned}
H(x, y, t) & \leq(100 n)^{2 n+2} \frac{1}{V\left(B_{\sqrt{t}}(y)\right)} \exp \left\{-\frac{d^{2}(x, y)}{100 t}\right\} \\
& \leq(100 n)^{2 n+2} \frac{1}{V\left(B_{3 r}(y)\right)} \cdot\left(\frac{3 r}{\sqrt{t}}\right)^{n} \exp \left\{-\frac{d^{2}(x, y)}{100 t}\right\} \\
& \leq(300 n)^{2 n+2} \frac{1}{V\left(B_{2 r}(p)\right)}\left(\frac{r}{\sqrt{t}}\right)^{n} \exp \left\{-\frac{r^{2}}{400 t}\right\} \\
& \leq(300 n)^{2 n+2} \frac{1}{V\left(B_{2 r}(p)\right)} \cdot(200 n)^{\frac{n}{2}} e^{-\frac{n}{2}} \\
& \leq(300 n)^{4 n} \frac{1}{V\left(B_{2 r}(p)\right)} .
\end{aligned}
$$

From (2.22), (2.23), (2.16) and (2.18), we get

$$
\begin{aligned}
& \frac{d}{d t} \int_{B_{2 r}(p)}(|\nabla \mathbf{b}|-1) \phi \cdot H(x, y, t) d x \\
\geq & -(400 n)^{4 n} f_{B_{\frac{7}{4} r}(p)}\left[|| \nabla \mathbf{b}|-1| \cdot \frac{1}{r^{2}}+\left|\nabla^{2} \mathbf{b}\right| \cdot \frac{1}{r}\right] \\
\geq & -(400 n)^{4 n}\left[2^{7 n}\left(\frac{r}{R}\right)^{\frac{1}{2(n-1)}} \frac{1}{r^{2}}+2^{37 n} r^{-2}\left(\frac{r}{R}\right)^{\frac{1}{(n-1)}}\right] \\
\geq & -\frac{2^{50 n^{2}}}{r^{2}} \cdot\left(\frac{r}{R}\right)^{\frac{1}{4(n-1)}} .
\end{aligned}
$$

Take the integration of (2.24), from (2.17) and (2.23), we get

$$
\begin{aligned}
|\nabla \mathbf{b}|(y)-1 & \leq \int_{B_{2 r}(p)}(|\nabla \mathbf{b}|-1) \phi \cdot H\left(x, y, r^{2}\right) d x+2^{50 n^{2}} \cdot\left(\frac{r}{R}\right)^{\frac{1}{4(n-1)}} \\
& \leq(300 n)^{4 n} f_{B_{\frac{7 r}{4}}(p)}|| \nabla \mathbf{b}|-1|+2^{50 n^{2}} \cdot\left(\frac{r}{R}\right)^{\frac{1}{4(n-1)}} \\
& \leq 2^{51 n^{2}}\left(\frac{r}{R}\right)^{\frac{1}{4(n-1)}} .
\end{aligned}
$$

On Riemannian manifolds, if there is a segment $\gamma_{p, q}$ between two points $p, q$, we can choose the middle point of the segment $\gamma_{p, q}$, denoted as $z$. Then $[p, q, z]$ is an AG-triple with the excess 0 and the scale $\frac{1}{2} d(p, q)$.

For a metric space $\mathbf{X}_{k}$, generally we can not find the middle point as in Riemannian manifolds. However, if there exists a suitable Gromov-Hausdorff approximation from $\mathbb{R}^{k} \times \mathbf{X}_{k}$ to manifold $M^{n}$ locally, the following lemma provides the existence of almost middle point and AG-triple in metric space $\mathbf{X}_{k}$.

Lemma 2.8. For $0 \leq k \leq n, 0<\delta<\frac{1}{3}, B_{10 r}(0, \hat{q}) \subset \mathbb{R}^{k} \times \mathbf{X}_{k}$ and $B_{10 r}(q) \subset\left(M^{n}, g\right)$, if there is an ( $\delta r)$-Gromov-Hausdorff approximation

$$
\Phi: B_{10 r}(0, \hat{q}) \rightarrow B_{10 r}(q)
$$


then for $\hat{q}_{1}^{+}, \hat{q}_{1}^{-} \in B_{r}(\hat{q}) \subset \mathbf{X}_{k}$, there exists $\hat{q}_{0} \in B_{3 r}(\hat{q})$ such that

$$
\left|d\left(\hat{q}_{0}, \hat{q}_{1}^{+}\right)-\frac{1}{2} d\left(\hat{q}_{1}^{+}, \hat{q}_{1}^{-}\right)\right| \leq 8 \sqrt{\delta} r \quad \text { and } \quad\left|d\left(\hat{q}_{0}, \hat{q}_{1}^{-}\right)-\frac{1}{2} d\left(\hat{q}_{1}^{+}, \hat{q}_{1}^{-}\right)\right| \leq 8 \sqrt{\delta} r .
$$

And if $\sqrt{\delta} r \leq \frac{d\left(\hat{q}_{1}^{+}, \hat{q}_{1}^{-}\right)}{100}$, then $\left[p_{k+1}^{+}, p_{k+1}^{-}, q_{1}\right]$ is an AG-triple with the excess $\leq 16 \sqrt{\delta} r$ and the scale $\geq \frac{1}{4} d\left(\hat{q}_{1}^{+}, \hat{q}_{1}^{-}\right)$, where

$$
q_{1}=\Phi\left(0, \hat{q}_{0}\right), \quad p_{k+1}^{+}=\Phi\left(0, \hat{q}_{1}^{+}\right), \quad p_{k+1}^{-}=\Phi\left(0, \hat{q}_{1}^{-}\right) .
$$

Proof: From the assumption that $\Phi$ is an $(\delta r)$-Gromov-Hausdorff approximation,

$$
\left|d\left(p_{k+1}^{+}, p_{k+1}^{-}\right)-d\left(\hat{q}_{1}^{+}, \hat{q}_{1}^{-}\right)\right|=\left|d\left(\Phi\left(0, \hat{q}_{1}^{+}\right), \Phi\left(0, \hat{q}_{1}^{-}\right)\right)-d\left(\left(0, \hat{q}_{1}^{+}\right),\left(0, \hat{q}_{1}^{-}\right)\right)\right|<\delta r .
$$

It is easy to see that $p_{k+1}^{+}, p_{k+1}^{-} \in B_{r+\delta r}(q)$. In fact the segment $\gamma_{p_{k+1}^{+}, p_{k+1}^{-}} \subset B_{3 r}(q)$, otherwise, note $d\left(\hat{q}_{1}^{+}, \hat{q}_{1}^{-}\right) \leq 2 r$, we will get

$$
d\left(p_{k+1}^{+}, p_{k+1}^{-}\right) \geq 2[3 r-(r+\delta r)]>d\left(\hat{q}_{1}^{+}, \hat{q}_{1}^{-}\right)+\delta r,
$$

which is contradicting (2.26).

Then we can choose the middle point of $\gamma_{p_{k+1}^{+}, p_{k+1}^{-}}$, denoted as $z_{0} \in B_{3 r}(q)$, then there exists $\left(s_{0}, \hat{q}_{0}\right) \in B_{10 r}(0, \hat{q})$ such that

$$
d\left(\Phi\left(s_{0}, \hat{q}_{0}\right), z_{0}\right)<\delta r .
$$

Now using $\frac{1}{2} d\left(p_{k+1}^{+}, p_{k+1}^{-}\right)=d\left(p_{k+1}^{+}, z_{0}\right)$ and (2.27), we have

$$
\begin{aligned}
& \left|d\left(\left(s_{0}, \hat{q}_{0}\right),\left(0, \hat{q}_{1}^{+}\right)\right)-\frac{1}{2} d\left(p_{k+1}^{+}, p_{k+1}^{-}\right)\right| \\
\leq & \left|d\left(\left(s_{0}, \hat{q}_{0}\right),\left(0, \hat{q}_{1}^{+}\right)\right)-d\left(\Phi\left(s_{0}, \hat{q}_{0}\right), p_{k+1}^{+}\right)\right| \\
& +\left|d\left(\Phi\left(s_{0}, \hat{q}_{0}\right), p_{k+1}^{+}\right)-d\left(p_{k+1}^{+}, z_{0}\right)\right| \\
< & \delta r+d\left(\Phi\left(s_{0}, \hat{q}_{0}\right), z_{0}\right)<2 \delta r .
\end{aligned}
$$

Similarly, by using $\frac{1}{2} d\left(p_{k+1}^{+}, p_{k+1}^{-}\right)=d\left(p_{k+1}^{-}, z_{0}\right)$, we have

$$
\left|d\left(\left(s_{0}, \hat{q}_{0}\right),\left(0, \hat{q}_{1}^{-}\right)\right)-\frac{1}{2} d\left(p_{k+1}^{+}, p_{k+1}^{-}\right)\right|<2 \delta r .
$$

From (2.26), (2.28) and (2.29),

$$
\begin{aligned}
& d\left(\hat{q}_{0}, \hat{q}_{1}^{+}\right)^{2}+s_{0}^{2}<\left(\frac{1}{2} d\left(p_{k+1}^{+}, p_{k+1}^{-}\right)+2 \delta r\right)^{2}<\left(\frac{1}{2} d\left(\hat{q}_{1}^{+}, \hat{q}_{1}^{-}\right)+\frac{5}{2} \delta r\right)^{2} \\
& d\left(\hat{q}_{0}, \hat{q}_{1}^{-}\right)^{2}+s_{0}^{2}<\left(\frac{1}{2} d\left(\hat{q}_{1}^{+}, \hat{q}_{1}^{-}\right)+\frac{5}{2} \delta r\right)^{2} .
\end{aligned}
$$


Take the sum of the above two inequalities, we have

$$
\begin{aligned}
2 \cdot\left(\frac{1}{2} d\left(\hat{q}_{1}^{+}, \hat{q}_{1}^{-}\right)+\frac{5}{2} \delta r\right)^{2} & >2 s_{0}^{2}+d\left(\hat{q}_{0}, \hat{q}_{1}^{+}\right)^{2}+d\left(\hat{q}_{0}, \hat{q}_{1}^{-}\right)^{2} \\
& \geq 2 s_{0}^{2}+\frac{1}{2}\left[d\left(\hat{q}_{0}, \hat{q}_{1}^{+}\right)+d\left(\hat{q}_{0}, \hat{q}_{1}^{-}\right)\right]^{2} \\
& \geq 2 s_{0}^{2}+\frac{1}{2} d\left(\hat{q}_{1}^{+}, \hat{q}_{1}^{-}\right)^{2}=\frac{1}{2} d\left(\hat{q}_{1}^{+}, \hat{q}_{1}^{-}\right)^{2}+2 s_{0}^{2} .
\end{aligned}
$$

Simplify the above inequality, using $d\left(\hat{q}_{1}^{+}, \hat{q}_{1}^{-}\right) \leq 2 r$, we get

$$
\left|s_{0}\right| \leq \frac{5}{2} \delta r+\sqrt{5} \sqrt{\delta} r \leq 5 \sqrt{\delta} r
$$

From (2.28), (2.29) and (2.30), we have

$$
\begin{aligned}
&\left|d\left(\left(0, \hat{q}_{0}\right),\left(0, \hat{q}_{1}^{+}\right)\right)-\frac{1}{2} d\left(p_{k+1}^{+}, p_{k+1}^{-}\right)\right|<7 \sqrt{\delta} r \\
&\left|d\left(\left(0, \hat{q}_{0}\right),\left(0, \hat{q}_{1}^{-}\right)\right)-\frac{1}{2} d\left(p_{k+1}^{+}, p_{k+1}^{-}\right)\right|<7 \sqrt{\delta} r .
\end{aligned}
$$

From (2.26), (2.31) and (2.32), we get (2.25) and the following

$$
\begin{aligned}
& d\left(q_{1}, p_{k+1}^{+}\right)+d\left(q_{1}, p_{k+1}^{-}\right)-d\left(p_{k+1}^{+}, p_{k+1}^{-}\right) \\
\leq & d\left(\left(0, \hat{q}_{0}\right),\left(0, \hat{q}_{1}^{+}\right)\right)+d\left(\left(0, \hat{q}_{0}\right),\left(0, \hat{q}_{1}^{-}\right)\right)-d\left(p_{k+1}^{+}, p_{k+1}^{-}\right)+2 \delta r \\
< & 14 \sqrt{\delta} r+2 \delta r \leq 16 \sqrt{\delta} r \\
d\left(q_{1}, p_{k+1}^{+}\right) \geq & d\left(\left(0, \hat{q}_{0}\right),\left(0, \hat{q}_{1}^{+}\right)\right)-\delta r>\frac{1}{2} d\left(p_{k+1}^{+}, p_{k+1}^{-}\right)-7 \sqrt{\delta} r-\delta r \\
\quad & \frac{1}{2}\left(d\left(\hat{q}_{1}^{+}, \hat{q}_{1}^{-}\right)-\delta r\right)-8 \sqrt{\delta} r \geq \frac{1}{4} d\left(\hat{q}_{1}^{+}, \hat{q}_{1}^{-}\right) .
\end{aligned}
$$

Similarly we have $d\left(q_{1}, p_{k+1}^{-}\right) \geq \frac{1}{4} d\left(\hat{q}_{1}^{+}, \hat{q}_{1}^{-}\right)$. Hence the scale is $\geq \frac{1}{4} d\left(\hat{q}_{1}^{+}, \hat{q}_{1}^{-}\right)$.

Finally from (2.25), we get

$$
d\left(\hat{q}_{0}, \hat{q}\right) \leq d\left(\hat{q}_{0}, \hat{q}_{1}^{+}\right)+d\left(\hat{q}, \hat{q}_{1}^{+}\right) \leq \frac{1}{2} d\left(\hat{q}_{1}^{+}, \hat{q}_{1}^{-}\right)+8 \sqrt{\delta} r+r<3 r,
$$

which implies $\hat{q}_{0} \in B_{3 r}(\hat{q})$.

Proposition 2.9. For $B_{10 r}(q) \subset\left(M^{n}, g\right)$ with $R c(g) \geq 0$ and any $\epsilon \in(0,1)$, any

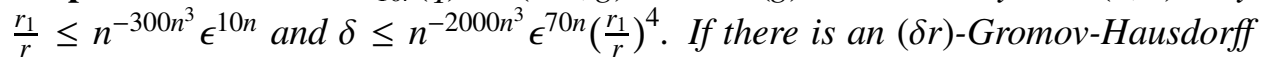
approximation for $0 \leq k \leq n$ and $B_{10 r}(0, \hat{q}) \subset \mathbb{R}^{k} \times \mathbf{X}_{k}$,

$$
\Phi: B_{10 r}(0, \hat{q}) \rightarrow B_{10 r}(q),
$$

where $\operatorname{diam}\left(B_{r}(\hat{q})\right)=r_{0} \geq \frac{1}{4} r$. Then there are harmonic functions $\left\{\mathbf{b}_{i}\right\}_{i=1}^{k+1}$ defined on some geodesic ball $B_{r_{1}}\left(q_{1}\right) \subset B_{10 r}(q)$, such that

$$
\sup _{\substack{B_{r_{1}\left(q_{1}\right)} \\ i=1, \cdots, k+1}}\left|\nabla \mathbf{b}_{i}\right| \leq 1+\epsilon \quad \text { and } \quad f_{B_{r_{1}}\left(q_{1}\right)} \sum_{i, j=1}^{k+1}\left|\left\langle\nabla \mathbf{b}_{i}, \nabla \mathbf{b}_{j}\right\rangle-\delta_{i j}\right| \leq \epsilon .
$$


Proof: We firstly assume $\delta \leq(400)^{-2}$, then from assumption $\operatorname{diam}\left(B_{r}(\hat{q})\right)=r_{0}$ and Lemma 2.8, there are $\hat{q}_{1}^{+}, \hat{q}_{1}^{-} \in B_{r}(\hat{q})$ and $\hat{q}_{0} \in B_{3 r}(\hat{q})$ such that

$$
\begin{aligned}
& d\left(\hat{q}_{1}^{+}, \hat{q}_{1}^{-}\right)=r_{0} \\
& \left|d\left(\hat{q}_{0}, \hat{q}_{1}^{+}\right)-\frac{1}{2} r_{0}\right| \leq 8 \sqrt{\delta} r \quad \text { and } \quad\left|d\left(\hat{q}_{0}, \hat{q}_{1}^{-}\right)-\frac{1}{2} r_{0}\right| \leq 8 \sqrt{\delta} r .
\end{aligned}
$$

Then we have

$$
\begin{aligned}
& \tilde{r}_{0}:=d\left(\hat{q}_{0}, \hat{q}_{1}^{+}\right) \geq \frac{1}{2} r_{0}-8 \sqrt{\delta} r \geq \frac{1}{4} r_{0} \geq \frac{1}{16} r, \\
& \tilde{r}_{0} \leq \frac{1}{2} r_{0}+8 \sqrt{\delta} r \leq 2 r .
\end{aligned}
$$

Let $\left\{\mathbf{e}_{i}\right\}_{i=1}^{k}$ be the standard basis for $\mathbb{R}^{k}$, put $q_{1}=\Phi\left(0, \hat{q}_{0}\right)$ and

$$
\begin{array}{cc}
p_{k+1}^{+}=\Phi\left(0, \hat{q}_{1}^{+}\right), & b_{k+1}^{+}(\cdot)=d\left(\cdot, p_{k+1}^{+}\right)-d\left(q_{1}, p_{k+1}^{+}\right), \\
p_{i}^{+}=\Phi\left(\tilde{r}_{0} \cdot \mathbf{e}_{i}, \hat{q}_{0}\right), & b_{i}^{+}(\cdot)=d\left(\cdot, p_{i}^{+}\right)-d\left(q_{1}, p_{i}^{+}\right) .
\end{array}
$$

From (2.33), (2.34), let $r_{1}=\zeta \cdot r$, if

$$
\begin{aligned}
& \zeta \leq n^{-110 n} \cdot \epsilon_{1}^{10 n} \\
& \delta \leq n^{-1250 n} \epsilon_{1}^{100 n} \zeta,
\end{aligned}
$$

then we can apply Proposition 1.10 to get

$$
f_{B_{r_{1}}\left(q_{1}\right)} \sum_{i, j=1}^{k+1}\left|\left\langle\nabla b_{i}^{+}, \nabla b_{j}^{+}\right\rangle-\delta_{i j}\right| \leq \epsilon_{1} .
$$

Define $p_{k+1}^{-}=\Phi\left(0, \hat{q}_{1}^{-}\right), p_{i}^{-}=\Phi\left(-\tilde{r}_{0} \cdot \mathbf{e}_{i}, \hat{q}_{0}\right)$, then from Lemma 2.8, $\left[p_{k+1}^{+}, p_{k+1}^{-}, q_{1}\right]$ is an AG-triple with the excess $\leq 16 \sqrt{\delta} r$ and the scale $\geq \frac{1}{4} r_{0}$. Because $\Phi$ is an $(\delta r)$ Gromov-Hausdorff approximation, it is easy to show that $\left[p_{i}^{+}, p_{i}^{-}, q_{1}\right]$ is AG-triple with the excess $\leq 16 \sqrt{\delta} r$ and the scale $\geq \frac{1}{4} r_{0}$ for $i=1, \cdots, k$.

If we assume

$$
\delta \leq \frac{\zeta^{4}}{n^{2}}
$$

then $16 \sqrt{\delta} r \leq \frac{4}{n} \frac{r_{1}^{2}}{\frac{1}{4} r_{0}}$. Also from $\frac{1}{4} r_{0} \geq \frac{1}{16} r$ and (2.35), we have $\frac{1}{4} r_{0} \geq 2^{2 n+1} r_{1}$.

Now we can apply Lemma 2.6 to obtain harmonic functions $\left\{\mathbf{b}_{i}\right\}_{i=1}^{k+1}$ satisfying

$$
\begin{aligned}
& \sup _{\substack{B_{r_{1}}\left(q_{1}\right) \\
i=1, \cdots, k+1}}\left|\nabla \mathbf{b}_{i}\right| \leq 1+2^{51 n^{2}}\left(\frac{r_{1}}{\frac{1}{4} r_{0}}\right)^{\frac{1}{4(n-1)}} \leq 1+2^{51 n^{2}} \epsilon_{1}^{2} \\
& \sup _{i=1, \cdots, k+1} f_{B_{r_{1}}\left(q_{1}\right)}\left|\nabla\left(\mathbf{b}_{i}-b_{i}^{+}\right)\right| \leq 2^{4 n}\left(\frac{r_{1}}{\frac{1}{4} r_{0}}\right)^{\frac{1}{2(n-1)}} \leq 2^{4 n} \epsilon_{1}^{5} .
\end{aligned}
$$


From 2.39) and 2.40), we get

$$
\begin{aligned}
& f_{B_{r_{1}}\left(q_{1}\right)}\left|\left\langle\nabla \mathbf{b}_{i}, \nabla \mathbf{b}_{j}\right\rangle-\delta_{i j}\right| \\
\leq & f_{B_{r_{1}}\left(q_{1}\right)}\left|\nabla\left(\mathbf{b}_{i}-b_{i}^{+}\right)\right| \cdot\left|\nabla \mathbf{b}_{j}\right|+\left|\left\langle\nabla b_{i}^{+}, \nabla\left(\mathbf{b}_{j}-b_{j}^{+}\right)\right\rangle\right|+\left|\left\langle\nabla b_{i}^{+}, \nabla b_{j}^{+}\right\rangle-\delta_{i j}\right| \\
\leq & \left(2+2^{51 n^{2}} \epsilon_{1}^{2}\right) \cdot 2^{4 n} \epsilon_{1}^{5}+f_{B_{r_{1}}\left(q_{1}\right)}\left|\left\langle\nabla b_{i}^{+}, \nabla b_{j}^{+}\right\rangle-\delta_{i j}\right| .
\end{aligned}
$$

From 2.37) and the above inequality, we have

$$
f_{B_{r_{1}}\left(q_{1}\right)} \sum_{i, j=1}^{k+1}\left|\left\langle\nabla \mathbf{b}_{i}, \nabla \mathbf{b}_{j}\right\rangle-\delta_{i j}\right| \leq(n+1)^{2} \cdot\left(2+2^{51 n^{2}} \epsilon_{1}^{2}\right) \cdot 2^{4 n} \epsilon_{1}^{5}+\epsilon_{1} .
$$

To get the conclusion, it is easy to check that $\epsilon_{1}=2^{-25 n^{2}} \epsilon$ is enough for the need.

From (2.35), 2.36) and (2.38), we get the conclusion.

Lemma 2.10. Suppose $\left(M^{n}, g\right)$ has $R c \geq 0$, for $z \in M^{n}$, let $f: B_{R}(z) \rightarrow \mathbb{R}$ be a nonnegative function and $f \in L^{1}\left(B_{R}(z)\right)$, then there exists $p \in B_{\frac{R}{2}}(z)$ such that

$$
\sup _{t \leq \frac{R}{2}} f_{B_{t}(p)} f \leq 15^{n} f_{B_{R}(z)} f .
$$

Proof: Let $c=15^{n} f_{B_{R}(z)} f$, define

$$
\mathcal{S}_{c}=\left\{x \in B_{R}(z): \sup _{B_{r}(x) \subset B_{R}(z)} f_{B_{r}(x)} f>c\right\},
$$

and $\mathcal{I}(f)(x)=\sup _{x \in B \subset B_{R}(z)} f_{B} f$, where the supremum is taken over all geodesic balls $B$ containing $x$ and $B \subset B_{R}(z)$.

Let $T_{c}=\left\{x \in B_{R}(z): \mathcal{I}(f)(x)>c\right\}$, then $\mathcal{S}_{c} \subset T_{c}$. Choose any $S \subset \subset T_{c}$, for any $x \in S$, there exists geodesic ball $B_{x} \subset B_{R}(z)$ such that $x \in B_{x}$ and

$$
V\left(B_{x}\right)<\frac{1}{c} \int_{B_{x}} f
$$

By compactness of $S$, we can select a finite collection of such balls $\left\{B_{\alpha}\right\}_{\alpha \in \mathrm{F}}$ that cover $S$. Set $R_{0}=\sup \left\{r_{\alpha} \mid \alpha \in \mathrm{F}\right\}$, and $\mathrm{F}_{j}=\left\{\alpha \in \mathrm{F} \mid \frac{R_{0}}{2^{j}}<r_{\alpha} \leq \frac{R_{0}}{2^{j-1}}\right\}, j=1,2, \cdots$.

We define $\mathrm{G}_{j} \subset \mathrm{F}_{j}$ as follows:

(1) Let $\mathrm{G}_{1}$ be any maximal collection of $\alpha$ in $\mathrm{F}_{1}$, such that $\left\{B_{r_{\alpha}}\left(x_{\alpha}\right)\right\}_{\alpha \in \mathrm{G}_{1}}$ are disjoint.

(2) Assume $G_{1}, \cdots, G_{k-1}$ have been selected, choose $G_{k}$ to be any maximal subcollection of

$$
\left\{\alpha \in \mathrm{F}_{k} \mid B_{r_{\alpha}}\left(x_{\alpha}\right) \cap B_{r_{\alpha^{\prime}}}\left(x_{\alpha^{\prime}}\right)=\emptyset \text { for any } \alpha^{\prime} \in \bigcup_{j=1}^{k-1} \mathrm{G}_{j}\right\},
$$

which also satisfies $B_{r_{\alpha}}\left(x_{\alpha}\right) \cap B_{r_{\beta}}\left(x_{\beta}\right)=\emptyset$ for any $\alpha, \beta \in G_{k}, \alpha \neq \beta$. 
Now we define $\mathrm{G}=\bigcup_{k=1}^{\infty} \mathrm{G}_{k} \subset \mathrm{F}$. For any $\alpha \in \mathrm{F}$, there is $j \in \mathbb{N}$, such that $\alpha \in \mathrm{F}_{j}$. If $\alpha \in \mathrm{G}_{j}$, we get $B_{r_{\alpha}}\left(x_{\alpha}\right) \subset \bigcup_{\alpha^{\prime} \in \mathrm{G}} B_{5 r_{\alpha^{\prime}}}\left(x_{\alpha^{\prime}}\right)$.

Otherwise $\alpha \notin \mathrm{G}_{j}$, by the definition of $\mathrm{G}_{j}$, there is $\alpha^{\prime} \in \bigcup_{i=1}^{j} \mathrm{G}_{i}$ such that $B_{r_{\alpha}}\left(x_{\alpha}\right) \cap B_{r_{\alpha^{\prime}}}\left(x_{\alpha^{\prime}}\right) \neq \emptyset$. Note $r_{\alpha} \leq \frac{R_{0}}{2^{j-1}}<2 r_{\alpha^{\prime}}$, hence $B_{r_{\alpha}}\left(x_{\alpha}\right) \subset B_{r_{\alpha^{\prime}}+2 r_{\alpha}}\left(x_{\alpha^{\prime}}\right) \subset$ $\bigcup_{\beta \in \mathrm{G}} B_{5 r_{\beta}}\left(x_{\beta}\right)$.

Then we find $\mathrm{G} \subset \mathrm{F}$, such that for any $\alpha^{\prime} \neq \beta^{\prime} \in \mathrm{G}, B_{r_{\alpha^{\prime}}}\left(x_{\alpha^{\prime}}\right) \cap B_{r_{\beta^{\prime}}}\left(x_{\beta^{\prime}}\right)=\emptyset$ and

$$
\bigcup_{\alpha \in \mathrm{F}} B_{r_{\alpha}}\left(x_{\alpha}\right) \subset \bigcup_{\alpha^{\prime} \in \mathrm{G}} B_{5 r_{\alpha^{\prime}}}\left(x_{\alpha^{\prime}}\right) \text {. }
$$

From Bishop-Gromov Comparison Theorem and (2.42), we get

$$
\begin{aligned}
\mu(S) & \leq \mu\left(\bigcup_{\alpha \in \mathrm{F}} B_{\alpha}\right) \leq \mu\left(\bigcup_{\alpha^{\prime} \in \mathrm{G}} B_{5 r_{\alpha^{\prime}}}\left(x_{\alpha^{\prime}}\right)\right) \leq 5^{n} \sum_{\alpha^{\prime} \in \mathrm{G}} \mu\left(B_{r_{\alpha^{\prime}}}\left(x_{\alpha^{\prime}}\right)\right) \\
& <5^{n} \cdot \sum_{\alpha^{\prime} \in \mathrm{G}}\left(\frac{1}{c} \int_{B_{r_{\alpha^{\prime}}}\left(x_{\alpha^{\prime}}\right)} f\right) \leq \frac{5^{n}}{c} \int_{B_{R}(z)} f .
\end{aligned}
$$

If one takes the supremum over all such $S \subset \subset T_{c}$, we have

$$
\mu\left(\mathcal{S}_{c}\right) \leq \mu\left(T_{c}\right) \leq \frac{5^{n}}{c} \int_{B_{R}(z)} f \leq 3^{-n} V\left(B_{R}(z)\right) .
$$

If (2.41) does not hold for any point in $B_{\frac{R}{2}}(z)$, then we have $B_{\frac{R}{2}}(z) \subset \mathcal{S}_{c}$, from Bishop-Gromov Volume Comparison Theorem, $\frac{\mu\left(\mathcal{S}_{c}\right)}{\mu\left(B_{R}(z)\right)} \geq \frac{\mu\left(B_{\frac{R}{2}}(z)\right)}{\mu\left(B_{R}(z)\right)} \geq 2^{-n}$, it is the contradiction, the conclusion follows.

The following lemma is well known so we omit its proof here.

Lemma 2.11. Let $\left(\mathbf{X}, d_{\mathbf{X}}, x_{0}\right)$ and $\left(\mathbf{Y}, d_{\mathbf{Y}}, y_{0}\right)$ be two pointed metric spaces, if there is a pointed $\epsilon$-Gromov-Hausdorff approximation $f:\left(\mathbf{X}, x_{0}\right) \rightarrow\left(\mathbf{Y}, y_{0}\right)$, then there exists a pointed $(3 \epsilon)$-Gromov-Hausdorff approximation $h:\left(\mathbf{Y}, y_{0}\right) \rightarrow\left(\mathbf{X}, x_{0}\right)$.

Theorem 2.12. For $B_{10 r}(q) \subset\left(M^{n}, g\right)$ with $R c(g) \geq 0$, any $0<\epsilon<1, \delta=$

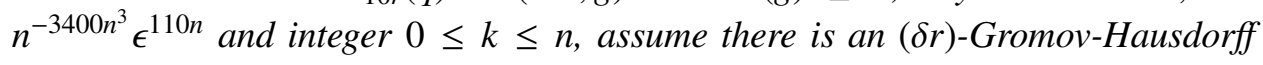
approximation ,

$$
f: B_{10 r}(q) \rightarrow B_{10 r}(0, \hat{q}) \subset \mathbb{R}^{k} \times \mathbf{X}_{k},
$$

and $\operatorname{diam}\left(B_{r}(\hat{q})\right)=r_{0} \geq \frac{1}{4} r$. Then there are harmonic functions $\left\{\mathbf{b}_{i}\right\}_{i=1}^{k+1}$ defined on some geodesic ball $B_{s}(p) \subset B_{10 r}(q)$, where $s=n^{-320 n^{3}} \epsilon^{10 n} r$, such that

$$
\sup _{\substack{B_{s}(p) \\ i=1, \cdots, k+1}}\left|\nabla \mathbf{b}_{i}\right| \leq 1+\epsilon \quad \text { and } \quad \sup _{t \leq s} f_{B_{t}(p)} \sum_{i, j=1}^{k+1}\left|\left\langle\nabla \mathbf{b}_{i}, \nabla \mathbf{b}_{j}\right\rangle-\delta_{i j}\right| \leq \epsilon .
$$

Proof: From Lemma 2.11, there is an (3 $\delta r)$-Gromov-Hausdorff approximation,

$$
\Phi: B_{10 r}(0, \hat{q}) \rightarrow B_{10 r}(q) \text {. }
$$


Let $r_{1}=n^{-300 n^{3}} \epsilon_{1}^{10 n} r$, assume $\delta \leq \frac{1}{3} n^{-3200 n^{3}} \epsilon_{1}^{110 n}$, where $\epsilon_{1}>0$ is to be determined later. Apply Proposition 2.9, we obtain harmonic functions $\left\{\mathbf{b}_{i}\right\}_{i=1}^{k+1}$ defined on $B_{r_{1}}\left(q_{1}\right) \subset B_{10 r}(q)$, such that

$$
\sup _{\substack{B_{r_{1}}\left(q_{1}\right) \\ i=1, \cdots, k+1}}\left|\nabla \mathbf{b}_{i}\right| \leq 1+\epsilon_{1} \quad \text { and } \quad f_{B_{r_{1}}\left(q_{1}\right)} \sum_{i, j=1}^{k+1}\left|\left\langle\nabla \mathbf{b}_{i}, \nabla \mathbf{b}_{j}\right\rangle-\delta_{i j}\right| \leq \epsilon_{1} .
$$

Apply Lemma2.10, we get $B_{s}(p) \subset B_{r_{1}}\left(q_{1}\right)$, where $s=\frac{r_{1}}{2}=\frac{1}{2} n^{-300 n^{3}} \epsilon_{1}^{10 n} r$, such that

$$
\sup _{\substack{B_{s}(p) \\ i=1, \cdots, k+1}}\left|\nabla \mathbf{b}_{i}\right| \leq 1+\epsilon_{1} \quad \text { and } \quad \sup _{t \leq s} f_{B_{t}(p)} \sum_{i, j=1}^{k+1}\left|\left\langle\nabla \mathbf{b}_{i}, \nabla \mathbf{b}_{j}\right\rangle-\delta_{i j}\right| \leq 15^{n} \epsilon_{1} .
$$

Choose $\epsilon_{1}=15^{-n} \epsilon$, let $\delta=n^{-3400 n^{3}} \epsilon^{110 n}$ and $s=n^{-320 n^{3}} \epsilon^{10 n} r$, the conclusion follows.

\section{PART II. A.O.L. HARMONIC FUNCTIONS PRODUCE G-H APPROXIMATION}

In Part II of this paper, we will prove the following quantitative version of almost splitting theorem, which was implied in the argument of Cheeger and Colding in a series of papers, [Col96a], [Col96b], [Col97], [CC96] and [Che99].

For our application, we need the quantitative estimate, which relate the GromovHausdorff distance to the average integral bound of almost orthonormal linear harmonic functions. Although we believe that many results in Part II are well-known to some experts in this field, but we can not find the reference providing those quantitative estimates exactly. So we elaborate the concise argument of CheegerColding to present the proof in all the details for self-contained reason.

Theorem 2.13. For $\epsilon>0$ and $1 \leq k \leq n$, there is $\delta=n^{-700 n^{4}} \epsilon^{18 n^{4}}$ such that for complete Riemannian manifold $\left(M^{n}, g\right)$ with $R c \geq 0$, if there exist harmonic functions $\left\{\mathbf{b}_{i}\right\}_{i=1}^{k}$, defined on $B_{r}(p)$, satisfying $\mathbf{b}_{i}(p)=0$ and

$$
\sup _{B_{r}(p)}\left|\nabla \mathbf{b}_{i}\right| \leq 2, \quad f_{B_{r}(p)} \sum_{i, j=1}^{k}\left|\left\langle\nabla \mathbf{b}_{i}, \nabla \mathbf{b}_{j}\right\rangle-\delta_{i j}\right| \leq \delta,
$$

then we can find a metric space $\mathbf{X}_{k}$ and an $(\epsilon s)$-Gromov-Hausdorff approximation $f_{k}=\left(\mathbf{b}_{1}, \cdots, \mathbf{b}_{k}, \mathcal{P}_{k}\right): B_{s}(p) \rightarrow B_{s}(0, \hat{p}) \subset \mathbb{R}^{k} \times \mathbf{X}_{k}$, where $s=\frac{1}{1280} r$.

Remark 2.14. Colding and Minicozzi [CMI14] gave a characterization of GromovHausdorff distance through the integral estimate of Hessian of harmonic functions among other things.

\section{Existence of almost linear function ANd Hessian estimate}

When there is a harmonic function $\mathbf{b}$ defined locally on manifold $M^{n}$, with bounded gradient and the average integral of ||$\nabla \mathbf{b}|-1|$ is small enough, we will 
show the existence of an almost linear function, which is a generalization of linear function in $\mathbb{R}^{n}$. The proof of Proposition 3.4 has close relationship with the argument in [Che99].

Definition 3.1. For $\mathbf{X} \subset M^{n}, t \in \mathbb{R}$, the function $\rho(x)=d(x, \mathbf{X})+t: M^{n} \rightarrow \mathbb{R}$ is called almost linear function, which is the generalization of function $f(x)=$ $d\left(x, \mathbb{R}^{n-1}\right)+t$ defined on $\mathbb{R}^{n}$.

Definition 3.2. For Lipschitz function $f$ defined on metric space $\mathbf{M}$, the pointwise Lipschitz constant function $\mathscr{L}(f)$ is defined by

$$
\mathscr{L}(f)(z)=\varlimsup_{d\left(z, z^{\prime}\right) \rightarrow 0} \frac{\left|f(z)-f\left(z^{\prime}\right)\right|}{d\left(z, z^{\prime}\right)}, \quad z, z^{\prime} \in \mathbf{M},
$$

and the Lipschtiz constant $\mathbf{L}(f)$ is defined by $\mathbf{L}(f)=\sup _{z \in \mathbf{M}}\{\mathscr{L}(f)(z)\}$.

Remark 3.3. From the classical Rademacher theorem, the Lipschitz function is almost differentiable on manifolds, for the general argument on metric measure spaces see [Che99]. Hence when the pointwise Lipschitz constant function $\mathscr{L}(f)(z)$ appears as the integrand function in an integral, we can replace it by $|\nabla f|(z)$, and we will use this fact freely in the following argument.

Proposition 3.4. For any $\epsilon>0$, there is $\delta=2^{-100 n^{2}} \epsilon^{2 n^{2}}$ such that for any complete Riemannian manifold $\left(M^{n}, g\right)$ with $R c \geq 0$, if there exists one harmonic function $\mathbf{b}$ defined on $B_{r}(p)$ satisfying $\sup _{B_{r}(p)}|\nabla \mathbf{b}| \leq 2$ and $f_{B_{r}(p)}|| \nabla \mathbf{b}|-1| \leq \delta$. Then one can find $t_{0}, t_{1} \in \mathbb{R}$ and two functions $\rho, \tilde{\rho}$ defined on $B_{\frac{r}{10}}(p)$, such that

$$
\begin{aligned}
& \rho(x)=d\left(x, \rho^{-1}\left(t_{0}\right)\right)+t_{0}, \quad \tilde{\rho}(x)=t_{1}-d\left(x, \tilde{\rho}^{-1}\left(t_{1}\right)\right), \quad \forall x \in B_{\frac{r}{160}}(p) \\
& \frac{1}{320} r \leq d\left(x, \rho^{-1}\left(t_{0}\right)\right) \leq \frac{3}{320} r, \quad \forall x \in B_{\frac{r}{320}}(p) \\
& \sup _{B_{\frac{1}{20} r}(p)}|\mathbf{b}-\rho| \leq \epsilon \cdot r, \quad f_{B_{\frac{1}{20} r}(p)}|\nabla(\mathbf{b}-\rho)| \leq \epsilon \\
& \rho(x) \leq \tilde{\rho}(x)+\frac{\epsilon}{2} r, \quad \forall x \in B_{\frac{1}{10}} r(p) .
\end{aligned}
$$

Proof: Step (1). For $1>\theta>0$ (to be determined later), we define

$$
\mathbf{A}=\left\{x \in B_{r}(p)|||\nabla \mathbf{b}(x)|-1 \mid<\frac{\delta}{\theta}\right\}
$$

then from assumption, we get

$$
V\left(B_{r}(p)-\mathbf{A}\right) \leq \theta \cdot V\left(B_{r}(p)\right) .
$$

We define $\mathbf{b}_{*}$ as the following:

$$
\mathbf{b}_{*}(x)=\left\{\begin{array}{l}
\mathbf{b}(x) \\
\sup _{z^{\prime} \in \mathbf{A}}\left[\mathbf{b}\left(z^{\prime}\right)-\left(1+\frac{\delta}{\theta}\right) \cdot d\left(x, z^{\prime}\right)\right]
\end{array} \quad x \in B_{r}(p)-\mathbf{A} .\right.
$$

It is easy to get $\sup _{x \in B_{r}(p)}\left|\mathscr{L}\left(\mathbf{b}_{*}\right)(x)\right| \leq 1+\frac{\delta}{\theta}$. 
Put $h(x)=\frac{\theta}{\delta+\theta} \mathbf{b}_{*}(x)+\frac{\delta}{\delta+\theta} \mathbf{b}_{*}(p)$, then

$$
\sup _{x \in B_{r}(p)}|\mathscr{L}(h)(x)|=\frac{\theta}{\delta+\theta} \sup _{x \in B_{r}(p)}\left|\mathscr{L}\left(\mathbf{b}_{*}\right)(x)\right| \leq 1 .
$$

Note $h(p)=\mathbf{b}_{*}(p)$ and $\sup _{B_{r}(p)}\left|\mathscr{L}\left(h-\mathbf{b}_{*}\right)\right| \leq \frac{\delta}{\theta}$, we get $\sup _{B_{r}(p)}\left|h-\mathbf{b}_{*}\right| \leq \frac{\delta}{\theta} r$.

For any $x \in \overline{B_{\frac{r}{5}}(p)}$, from $\left(\overline{3.2)}\right.$, there exists $x^{\prime} \in B_{\frac{7}{5}} \theta^{\frac{1}{n}} r(x)$ such that $x^{\prime} \in \mathbf{A}$. Note $\mathbf{b}\left(x^{\prime}\right)-\mathbf{b}_{*}\left(x^{\prime}\right)=0$ and

$$
\sup _{B_{r}(p)}\left|\mathscr{L}\left(\mathbf{b}-\mathbf{b}_{*}\right)\right| \leq \sup _{B_{r}(p)}|\nabla \mathbf{b}|+\sup _{B_{r}(p)}\left|\mathscr{L}\left(\mathbf{b}_{*}\right)\right| \leq 3+\frac{\delta}{\theta} .
$$

Then for any $x \in \overline{B_{\frac{r}{5}}(p)}$, we have

$$
\left|\mathbf{b}(x)-\mathbf{b}_{*}(x)\right| \leq\left|\mathbf{b}\left(x^{\prime}\right)-\mathbf{b}_{*}\left(x^{\prime}\right)\right|+\left(3+\frac{\delta}{\theta}\right) \cdot \frac{7}{5} \theta^{\frac{1}{n}} r=\left(3+\frac{\delta}{\theta}\right) \cdot \frac{7}{5} \theta^{\frac{1}{n}} r .
$$

From above we have

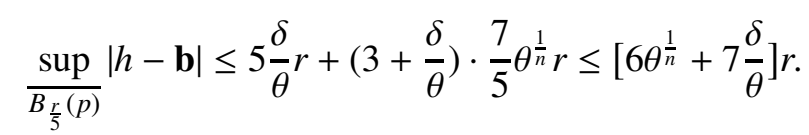

From (3.2), note $h=\frac{\theta}{\delta+\theta} \mathbf{b}+\frac{\delta}{\delta+\theta} \mathbf{b}_{*}(p)$ on $\mathbf{A}$, we have

$$
\begin{aligned}
f_{B_{\frac{r}{5}}(p)}(1 & -\langle\nabla \mathbf{b}, \nabla h\rangle) \leq \frac{1}{V\left(B_{\frac{r}{5}}(p)\right)}\left[\int_{B_{\frac{r}{5}}(p)-\mathbf{A}} 3+\int_{\mathbf{A} \cap B_{\frac{r}{5}}(p)}(1-\langle\nabla \mathbf{b}, \nabla h\rangle)\right] \\
& \leq 3 \frac{V\left(B_{\frac{r}{5}}(p)-\mathbf{A}\right)}{V\left(B_{\frac{r}{5}}(p)\right)}+\frac{1}{V\left(B_{\frac{r}{5}}(p)\right)} \int_{\mathbf{A} \cap B_{\frac{r}{5}}(p)}\left\{1-\left\langle\nabla \mathbf{b}, \frac{\theta}{\delta+\theta} \nabla \mathbf{b}\right\rangle\right\} \\
& \leq 3 \theta \frac{V\left(B_{r}(p)\right)}{V\left(B_{\frac{r}{5}}(p)\right)}+\frac{1}{V\left(B_{\frac{r}{5}}(p)\right)} \int_{\mathbf{A} \cap B_{\frac{r}{5}}(p)}\left\{\frac{\delta}{\delta+\theta}+\frac{\theta}{\delta+\theta}\left(1-|\nabla \mathbf{b}|^{2}\right)\right\} \\
& \leq 3 \cdot 5^{n} \theta+\frac{4 \delta}{\theta+\delta} \leq 4\left(5^{n} \theta+\frac{\delta}{\theta}\right) .
\end{aligned}
$$

Now from (3.3) and (3.4), we can choose $\theta=2^{-12 n^{2}} \epsilon_{1}^{n}$, where $\epsilon_{1}>0$ is to be determined later, and

$$
\delta \leq 2^{-20 n^{2}} \epsilon_{1}^{n+1} .
$$

Then we have

$$
\frac{\sup }{B \frac{r}{5}(p)}|h-\mathbf{b}| \leq \epsilon_{1} r \quad \text { and } \quad f_{B_{\frac{r}{5}}(p)}(1-\langle\nabla \mathbf{b}, \nabla h\rangle) \leq \epsilon_{1} .
$$

Step (2). Now for $x \in \overline{B_{\frac{1}{5}}(p)}$, we consider

$$
\begin{aligned}
& \tilde{\rho}(x)=\sup _{z^{\prime} \in \partial B_{\frac{1}{r}}(p)}\left[h\left(z^{\prime}\right)-d\left(x, z^{\prime}\right)\right], \\
& \rho(x)=\inf _{z^{\prime} \in \partial B_{\frac{1}{5} r}(p)}\left[h\left(z^{\prime}\right)+d\left(x, z^{\prime}\right)\right] .
\end{aligned}
$$


For $x \in B_{\frac{r}{10}}(p)$, assume

$$
\rho(x)=h\left(x^{*}\right)+d\left(x^{*}, x\right),
$$

where $x^{*} \in \partial B_{\frac{r}{5}}(p)$. Let $\gamma_{x, x^{*}}(s)$ be the minimizing geodesic from $x$ to $x^{*}$, parametrized by arc-length. From $\mathbf{L}(\rho) \leq 1$, we get

$\rho\left(\gamma_{x, x^{*}}(s)\right) \geq \rho(x)-s=h\left(x^{*}\right)+d\left(x, x^{*}\right)-s=h\left(x^{*}\right)+d\left(\gamma_{x, x^{*}}(s), x^{*}\right) \geq \rho\left(\gamma_{x, x^{*}}(s)\right)$,

which implies

$$
\rho\left(\gamma_{x, x^{*}}(s)\right)=\rho(x)-s, \quad \forall s \in\left[0, d\left(x, x^{*}\right)\right] .
$$

But $d\left(x, x^{*}\right) \geq \frac{r}{10}$, we have

$$
\rho\left(\gamma_{x, x^{*}}(s)\right)=\rho(x)-s, \quad \forall s \in\left[0, \frac{r}{10}\right] .
$$

Let $t_{0}=\rho(p)-\frac{r}{160}$, note $0 \leq \rho(x)-t_{0} \leq \frac{r}{10}$ for $x \in B_{\frac{r}{160}}(p)$, then from (3.7),

$$
t_{0}=\rho(x)-\left[\rho(x)-t_{0}\right]=\rho\left(\gamma_{x, x^{*}}\left(\rho(x)-t_{0}\right)\right) .
$$

Now we have

$$
\rho(x)-t_{0} \leq d\left(x, \rho^{-1}\left(t_{0}\right)\right) \leq d\left(x, \gamma_{x, x^{*}}\left(\rho(x)-t_{0}\right)\right)=\rho(x)-t_{0} .
$$

By the above, for $x \in B_{\frac{r}{160}}(p)$, we get

$$
\rho(x)=t_{0}+d\left(x, \rho^{-1}\left(t_{0}\right)\right) \text {. }
$$

Similarly, let $t_{1}=\tilde{\rho}(p)+\frac{r}{160}$, for $x \in B_{\frac{r}{160}}(p)$, we get

$$
\tilde{\rho}(x)=t_{1}-d\left(x, \tilde{\rho}^{-1}\left(t_{1}\right)\right) \text {. }
$$

For any $x \in B_{\frac{r}{320}}(p)$, using $\mathbf{L}(\rho) \leq 1$, we have

$$
d\left(x, \rho^{-1}\left(t_{0}\right)\right)=\rho(x)-t_{0}=\rho(x)-\rho(p)+\frac{r}{160} \in\left[\frac{r}{320}, \frac{3 r}{320}\right] .
$$

Step (3). Let $\epsilon_{2}=2^{11} \epsilon_{1}^{\frac{1}{n}}$, we will prove that

$$
\rho(w) \leq \tilde{\rho}(w)+\epsilon_{2} r, \quad \forall w \in B_{\frac{1}{10} r}(p)
$$

By contradiction, if there exists $w \in B_{\frac{1}{10}} r(p)$ such that

$$
\tilde{\rho}(w)+2 \theta_{1} r \leq \rho(w),
$$

where $\theta_{1}=2{ }^{10} \epsilon_{1}^{\frac{1}{n}} \leq \frac{1}{20}$. Let $K=\frac{1}{2}[\tilde{\rho}(w)+\rho(w)]$, since $|\mathbf{L}(\rho)| \leq 1,|\mathbf{L}(\tilde{\rho})| \leq 1$ and $\tilde{\rho} \leq \rho$, also note $B_{\theta r}(w) \subset B_{\frac{1}{5} r}(p)$, we have

$$
\tilde{\rho}(x) \leq K \leq \rho(x), \quad x \in B_{\theta_{1} r}(w) .
$$

Now we define

$$
\check{h}(x)=\left\{\begin{array}{lc}
\rho(x), & \text { if } \rho(x) \leq K \\
K, & \text { if } \tilde{\rho}(x) \leq K \leq \rho(x) \\
\tilde{\rho}(x), & \text { if } K \leq \tilde{\rho}(x),
\end{array}\right.
$$


then $|\mathbf{L}(\breve{h})| \leq 1$, also note $\breve{h}=\tilde{\rho}=\rho=h$ on $\partial B_{\frac{1}{5} r}(p)$, from (3.6), we get

$$
\sup _{\partial B_{\frac{1}{5} r}(p)}|\check{h}-\mathbf{b}|=\sup _{\partial B_{\frac{1}{5} r}(p)}|h-\mathbf{b}| \leq \epsilon_{1} r .
$$

We define the Lipschitz function $\phi(x): \overline{B_{\frac{1}{5} r}(p)} \rightarrow \mathbb{R}$ satisfying

$$
0 \leq \phi(x) \leq 1 \quad \text { and } \quad \mathbf{L}(\phi) \leq\left(\epsilon_{1} r\right)^{-1},
$$

also

$$
\phi(x)= \begin{cases}0 & x \in \partial B_{r}(p) \\ 1 & x \in B_{\left(\frac{1}{5}-\epsilon_{1}\right) r}(p) .\end{cases}
$$

Now define $\tilde{\mathbf{b}}=\breve{h}+(1-\phi)(\mathbf{b}-\check{h})$, we have

$$
\begin{aligned}
|\mathbf{L}[(1-\phi) \cdot(\mathbf{b}-\check{h})]| & \leq \sup _{B_{\frac{r}{5}}(p)}(1-\phi) \cdot|\mathscr{L}(\mathbf{b}-\check{h})|+\sup _{B_{\frac{r}{5}}(p) \backslash B_{\left(\frac{1}{5}-\epsilon_{1}\right) r}(p)}|\mathbf{b}-\check{h}| \cdot|\mathscr{L}(1-\phi)| \\
& \leq[|\nabla \mathbf{b}|+|\mathbf{L}(\breve{h})|]+\left[\sup _{\partial B_{\frac{r}{5}}(p)}|\mathbf{b}-\breve{h}|+\left(\epsilon_{1} r\right) \cdot \sup _{B_{\frac{r}{5}}(p)}|\mathscr{L}(\mathbf{b}-\breve{h})|\right] \cdot\left(\epsilon_{1} r\right)^{-1} \\
& \leq 3+\left[\epsilon_{1} r+3 \epsilon_{1} r\right]\left(\epsilon_{1} r\right)^{-1}=7 .
\end{aligned}
$$

Let $\chi_{E}$ denote the characteristic function of a set $E$, then from above and the Bishop-Gromov Comparison Theorem we get

$$
\begin{aligned}
f_{B_{\frac{r}{5}}(p)}|\nabla \tilde{\mathbf{b}}|^{2} & \leq f_{B_{\frac{r}{5}(p)}}(|\nabla \breve{h}|+|\nabla[(1-\phi) \cdot(\mathbf{b}-\check{h})]|)^{2} \leq f_{B_{\frac{r}{5}}(p)}\left(|\nabla \breve{h}|+7 \chi_{B_{\frac{r}{5}} \backslash B_{\left(\frac{1}{5}-\epsilon_{1}\right) r}}\right)^{2} \\
& \leq f_{B_{\frac{r}{5}(p)}}|\nabla \check{h}|^{2}+70\left(1-\frac{V\left(B_{\left(\frac{1}{5}-\epsilon_{1}\right) r}(p)\right)}{V\left(B_{\frac{r}{5}}(p)\right)}\right) \leq f_{B_{\frac{r}{5}}(p)}|\nabla \check{h}|^{2}+70\left(1-\left(1-5 \epsilon_{1}\right)^{n}\right) \\
& \leq 350 n \cdot \epsilon_{1}+f_{B_{\frac{r}{5}}(p)}|\nabla \check{h}|^{2} .
\end{aligned}
$$

From the fact that $\left.\tilde{\mathbf{b}}\right|_{\partial B_{\frac{r}{5}(p)}}=\left.\mathbf{b}\right|_{\partial B_{\frac{r}{5}}(p)}$ and $\mathbf{b}$ is harmonic, note the harmonic function has the smallest energy, we have

$$
f_{\frac{r}{5}(p)}|\nabla \mathbf{b}|^{2} \leq f_{B_{\frac{r}{5}}(p)}|\nabla \tilde{\mathbf{b}}|^{2} \leq 350 n \epsilon_{1}+f_{B_{\frac{r}{5}}(p)}|\nabla \check{h}|^{2},
$$

which implies

$$
f_{B_{\frac{r}{5}(p)}}\left[1-|\nabla \check{h}|^{2}\right] \leq 350 n \epsilon_{1}+f_{B_{\frac{r}{5}(p)}}\left[1-|\nabla \mathbf{b}|^{2}\right] \leq 350 n \epsilon_{1}+3 \cdot 5^{n} \delta \leq 400 n \epsilon_{1} .
$$

In the last inequality above, we used (3.5).

On the other hand, note $\mathscr{L}(\breve{h})=0$ on $B_{\theta_{1} r}(w)$, we have

$400 n \epsilon_{1} \geq f_{B_{\frac{r}{5}}(p)}\left[1-|\nabla \check{h}|^{2}\right] \geq \frac{1}{V\left(B_{\frac{r}{5}}(p)\right)} \int_{B_{\theta_{1} r}(w)}\left[1-|\nabla \check{h}|^{2}\right]=\frac{V\left(B_{\theta_{1} r}(w)\right)}{V\left(B_{\frac{r}{5}}(p)\right)} \geq\left(\frac{10 \theta_{1}}{3}\right)^{n}$,

which implies $\theta_{1}<2^{10} \epsilon_{1}^{\frac{1}{n}}$, it is the contradiction with the choice of $\theta_{1}$. 
Step (4). Note $\tilde{\rho}(x) \leq h(x) \leq \rho(x)$ and (3.9), then we get

$$
|h(x)-\rho(x)| \leq \rho(x)-\tilde{\rho}(x) \leq \epsilon_{2} r, \quad \forall x \in B_{\frac{r}{20}}(p) .
$$

From (3.6) and (3.10) we have

$$
\sup _{B_{\frac{1}{20} r}(p)}|\mathbf{b}-\rho| \leq\left(2^{11}+1\right) \epsilon_{1}^{\frac{1}{n}} r \leq 2^{12} \epsilon_{1}^{\frac{1}{n}} r .
$$

From (3.5) and (3.6),

$$
\begin{aligned}
& f_{B_{\frac{1}{20} r}(p)}|\nabla(\mathbf{b}-\rho)|^{2} \\
\leq & 4^{n} f_{B_{\frac{1}{5} r}(p)}|\nabla(\mathbf{b}-\rho)|^{2}=4^{n} f_{B_{\frac{1}{5} r}(p)}|\nabla \mathbf{b}|^{2}+|\nabla \rho|^{2}-2\langle\nabla \mathbf{b}, \nabla \rho\rangle \\
\leq & 4^{n} f_{B_{\frac{1}{5} r}(p)}\left(|\nabla \mathbf{b}|^{2}-1\right)+\left(|\nabla \rho|^{2}-1\right)+2(1-\langle\nabla \mathbf{b}, \nabla h\rangle)+2\langle\nabla \mathbf{b}, \nabla(h-\rho)\rangle \\
\leq & 4^{n}\left[3 \cdot 5^{n} \delta+0+2 \epsilon_{1}+0\right]=4^{n} \cdot\left(3 \epsilon_{1}\right),
\end{aligned}
$$

then

$$
f_{B_{\frac{1}{20} r}(p)}|\nabla(\mathbf{b}-\rho)| \leq\left(f_{B_{\frac{1}{20} r}(p)}|\nabla(\mathbf{b}-\rho)|^{2}\right)^{\frac{1}{2}} \leq 2^{n+1} \sqrt{\epsilon_{1}} .
$$

If we choose $\epsilon_{1} \leq 2^{-23 n} \epsilon^{n}$ and also $2^{10} \epsilon_{1}^{\frac{1}{n}} \leq \frac{1}{20}$, then we obtain the conclusion. From (3.5), we only need to choose $\delta \leq 2^{-100 n^{2}} \epsilon^{2 n^{2}}$.

\section{Segment INequality AND MEASURe of 'GOOD' POINTS}

In the proofs of this section, when the context is clear, for simplicity, we use $B_{r}$ instead of $B_{r}(p)$, similar for $B_{4 r}$ etc.

Lemma 4.1 (Segment Inequality). Assume $\left(M^{n}, g\right)$ is a complete Riemannian manifold with $R c \geq 0$, then for any nonnegative function $f$ defined on $B_{2 r}(p) \subset M^{n}$,

$$
\int_{B_{r}(p) \times B_{r}(p)}\left(\int_{0}^{d\left(y_{1}, y_{2}\right)} f\left(\gamma_{y_{1}, y_{2}}(s)\right) d s\right) d y_{1} d y_{2} \leq 2^{n+1} r \cdot V\left(B_{r}(p)\right) \cdot \int_{B_{2 r}(p)} f .
$$

Proof: In the proof, we assume $y_{1}, y_{2} \in B_{r}$, and set

$$
\begin{aligned}
& E\left(y_{1}, y_{2}\right)=\int_{0}^{d\left(y_{1}, y_{2}\right)} f\left(\gamma_{y_{1}, y_{2}}(s)\right) d s \\
& E_{1}\left(y_{1}, y_{2}\right)=\int_{\frac{1}{2} d\left(y_{1}, y_{2}\right)}^{d\left(y_{1}, y_{2}\right)} f\left(\gamma_{y_{1}, y_{2}}(s)\right) d s, \quad E_{2}\left(y_{1}, y_{2}\right)=\int_{0}^{\frac{1}{2} d\left(y_{1}, y_{2}\right)} f\left(\gamma_{y_{1}, y_{2}}(s)\right) d s .
\end{aligned}
$$

Then $E=E_{1}+E_{2}$. Along any geodesic $\gamma$ starting from $y_{1}$, write the volume element of $M^{n}$ in geodesic polar coordinate as $d s \wedge \mathcal{A}(s)$. Then $\mathcal{A}(s)=J(\theta, s) d \theta$, from Bishop-Gromov Comparison Theorem,

$$
\mathcal{A}(s) \leq\left(\frac{s}{u}\right)^{n-1} \mathcal{A}(u) \leq 2^{n-1} \cdot \mathcal{A}(u), \quad \forall u \in\left[\frac{s}{2}, s\right] .
$$


For $y \in B_{r}, v \in S_{y} M^{n}$, we define

$$
I(y, v)=\left\{t \geq 0\left|\gamma(t) \in B_{r}, \gamma^{\prime}(0)=v, \gamma\right|_{[0, t]} \text { is minimal }\right\} .
$$

Then we have

$$
\sup _{y \in B_{r}, v \in S_{y} M^{n}}|I(y, v)| \leq 2 r
$$

where $|I(y, v)|$ denotes the measure of $I(y, v)$.

Assume $\gamma_{y_{1}}^{v_{1}}$ is the geodesic starting from $y_{1}$ with $\left(\gamma_{y_{1}}^{\nu_{1}}\right)^{\prime}(0)=v_{1}$, then for any $y_{1} \in B_{r}, s \in I\left(y_{1}, v_{1}\right)$,

$$
\begin{aligned}
E_{1}\left(y_{1}, \gamma_{y_{1}}^{v_{1}}(s)\right) \mathcal{A}(s) & =\mathcal{A}(s) \int_{\frac{1}{2} s}^{s} f\left(\gamma_{y_{1}}^{\nu_{1}}(u)\right) d u \leq 2^{n-1} \cdot \int_{\frac{1}{2} s}^{s} f\left(\gamma_{y_{1}}^{\nu_{1}}(u)\right) \mathcal{A}(u) d u \\
& \leq 2^{n-1} \cdot \int_{0}^{\mathcal{T}\left(y_{1}, v_{1}\right)} f\left(\gamma_{y_{1}}^{\nu_{1}}(t)\right) \mathcal{A}(t) d t
\end{aligned}
$$

where $\mathcal{T}\left(y_{1}, v_{1}\right)=\max _{t \in I\left(y_{1}, v_{1}\right)} t$.

Thus from 4.1], for any $y_{1} \in B_{r}, v_{1} \in S_{y_{1}} M^{n}$,

$$
\int_{I\left(y_{1}, v_{1}\right)} E_{1}\left(y_{1}, \gamma_{y_{1}}^{v_{1}}(s)\right) \mathcal{A}(s) d s \leq 2^{n} r \cdot \int_{0}^{\mathcal{T}\left(y_{1}, v_{1}\right)} f\left(\gamma_{y_{1}}^{v_{1}}(t)\right) \mathcal{A}(t) d t .
$$

Integrating (4.2) with respect to $v_{1}$ over the unit tangent space $S_{y_{1}} M^{n}$, and note $\left(\bigcup_{\substack{y_{1} \in B_{r}(p) \\ y_{2} \in B_{r}(p)}} \gamma_{y_{1}, y_{2}}\right) \subset B_{2 r}$, where $\gamma_{y_{1}, y_{2}}$ is the minimal geodesic connecting $y_{1}$ with $y_{2}$ in $M^{n}$, we have

$$
\int_{B_{r}} E_{1}\left(y_{1}, y_{2}\right) d y_{2} \leq 2^{n} r \cdot \int_{B_{2 r}} f, \quad \forall y_{1} \in B_{r} .
$$

And we integrate (4.3) with respect to $y_{1}$ over $B_{r}$,

$$
\int_{B_{r} \times B_{r}} \int_{\frac{1}{2} d\left(y_{1}, y_{2}\right)}^{d\left(y_{1}, y_{2}\right)} f\left(\gamma_{y_{1}, y_{2}}(s)\right) d s=\int_{B_{r} \times B_{r}} E_{1}\left(y_{1}, y_{2}\right) \leq 2^{n} r V\left(B_{r}\right) \cdot \int_{B_{2 r} r} f .
$$

Similarly, we get

$$
\int_{B_{r} \times B_{r}} \int_{0}^{\frac{1}{2} d\left(y_{1}, y_{2}\right)} f\left(\gamma_{y_{1}, y_{2}}(s)\right) d s=\int_{B_{r} \times B_{r}} E_{2}\left(y_{1}, y_{2}\right) \leq 2^{n} r V\left(B_{r}\right) \cdot \int_{B_{2 r}} f .
$$

Take the sum of (4.4) and (4.5), the conclusion follows.

For $x \in B_{2 r}(p) \subset M^{n}$ and a closed subset $\mathbf{X} \subseteq M^{n}$, we define

$$
\rho(x)=d(x, \mathbf{X})+t_{0}, \quad \hat{\rho}(x)=d(x, \mathbf{X}),
$$

where $t_{0} \in \mathbb{R}$ is some constant, we define $\mathfrak{P}(x) \in \mathbf{X}$ by $d(x, \mathbf{X})=d(x, \mathfrak{P}(x))$ (if there are two points $y_{1}, y_{2}$ satisfying $d(x, \mathbf{X})=d\left(x, y_{1}\right)=d\left(x, y_{2}\right)$, then define $\mathfrak{P}(x)=y_{1}$ or $y_{2}$ freely). We assume

$$
0<r \leq \hat{\rho}(x) \leq 3 r, \quad \forall x \in B_{r}(p) .
$$


For $0<\eta<\frac{1}{2}$, we have $\left|\frac{\hat{\rho}(y)-\hat{\rho}(x)}{\hat{\rho}(x)-\eta r}\right| \leq 4$. And we also define

$$
\mathfrak{G}_{\rho}(x)=(\hat{\rho}(x), \mathfrak{P}(x)): B_{r}(p) \rightarrow \mathbb{R} \times \mathbf{X} .
$$

For $x, y \in B_{r}(p)$, define

$$
\begin{array}{lr}
\sigma_{x}(s)=\gamma_{\mathfrak{P}(x), x}(s+\eta r), & \tilde{\sigma}_{y}(s)=\sigma_{y}\left(\frac{\hat{\rho}(y)-\eta r}{\hat{\rho}(x)-\eta r} s\right)=\gamma_{\mathfrak{P}(y), y}\left(\frac{\hat{\rho}(y)-\eta r}{\hat{\rho}(x)-\eta r} s+\eta r\right) \\
\tau_{s}=\gamma_{\sigma_{x}(s), \tilde{\sigma}_{y}(s)}, & l_{s}=d\left(\sigma_{x}(s), \tilde{\sigma}_{y}(s)\right) .
\end{array}
$$

Definition 4.2. For $0<\eta<\frac{1}{2}$, we define

$$
\begin{aligned}
Q_{\eta, \mathbf{b}}^{r, \rho} & =\left\{x \in B_{r}(p): \int_{0}^{\hat{\rho}(x)-\eta r}|\nabla \mathbf{b}-\nabla \rho|\left(\sigma_{x}(s)\right) d s \leq \eta r\right\} \\
T_{\eta, \mathbf{b}}^{r, \rho} & =\left\{x \in B_{r}(p): f_{B_{r}(p)} d y\left(\int_{0}^{\hat{\rho}(x)-\eta r}\left(\int_{\gamma_{\sigma_{x}(s), \tilde{\sigma} y(s)}}\left|\nabla^{2} \mathbf{b}\right|\right) d s\right) \leq \eta r\right\}, \\
T_{\eta, \mathbf{b}}^{r, \rho}(x) & =\left\{y \in B_{r}(p): \int_{0}^{\hat{\rho}(x)-\eta r}\left(\int_{\gamma_{\sigma_{x}(s), \tilde{\sigma} y(s)}}\left|\nabla^{2} \mathbf{b}\right|\right) d s \leq \sqrt{\eta} r\right\}, \quad \forall x \in T_{\eta, \mathbf{b}}^{r, \rho} .
\end{aligned}
$$

For non-negative function $f$ defined on $B_{r}(p)$, we define

$$
\mathfrak{Q}_{\eta, f}^{r, \rho}:=\left\{x \in B_{r}(p): \int_{0}^{\hat{\rho}(x)-\eta r} f\left(\sigma_{x}(s)\right) d s \leq \eta r\right\} .
$$

Lemma 4.3. Assume (4.6), then for any non-negative function $f$ satisfying $f_{B_{4 r}(p)} f \leq$ $\delta$, we have $\frac{V\left(\mathfrak{Q}_{\eta, f}^{r, \rho}\right)}{V\left(B_{r}(p)\right)} \geq 1-3^{n} \eta^{-n} \delta$.

Proof: Firstly we have

$$
\int_{B_{r} \backslash \mathfrak{Q}_{\eta, f}^{r, \rho}} d x \int_{0}^{\hat{\rho}(x)-\eta r} f\left(\sigma_{x}(s)\right) d s \geq \eta r \cdot V\left(B_{r} \backslash \mathfrak{Q}_{\eta, f}^{r, \rho}\right) .
$$

Assume $\theta_{s}(x)$ is the gradient flow of $\rho(\cdot)$ starting from $\mathfrak{P}(x)$ at time $s+\eta r$, using Co-Area formula and Bishop-Gromov volume comparison theorem,

$$
\begin{aligned}
& \int_{B_{r} \backslash Q_{\eta, f}^{r, \rho}} d x \int_{0}^{\hat{\rho}(x)-\eta r} f\left(\sigma_{x}(s)\right) d s \leq \int_{B_{r}} d x \int_{0}^{\hat{\rho}(x)-\eta r} f\left(\sigma_{x}(s)\right) d s \\
= & \int_{r}^{3 r} d t \int_{\hat{\rho}^{-1}(t) \cap B_{r}} d x \int_{0}^{t-\eta r} f\left(\theta_{s}(x)\right) d s=\int_{r}^{3 r} d t \int_{0}^{t-\eta r} d s \int_{\hat{\rho}^{-1}(t) \cap B_{r}} f\left(\theta_{s}(x)\right) d x \\
\leq & \left(\frac{3 r}{\eta r}\right)^{n-1} \int_{r}^{3 r} d t \int_{0}^{t-\eta r} d s \int_{\theta_{s}\left(\hat{\rho}^{-1}(t) \cap B_{r}\right)} f(\tilde{x}) d \tilde{x} \leq\left(\frac{3}{\eta}\right)^{n-1} \int_{r}^{3 r} d t \int_{B_{4 r}} f(\tilde{x}) d \tilde{x} \\
\leq & \left(\frac{3}{\eta}\right)^{n-1} \cdot 2 r \cdot f_{B_{4 r}} f \cdot V\left(B_{4 r}\right) \leq 3^{n} \eta^{1-n} \delta r \cdot V\left(B_{r}\right) .
\end{aligned}
$$

From the above, we obtain $\frac{V\left(\mathbb{2}_{\eta, f}^{r, \rho}\right)}{V\left(B_{r}\right)} \geq 1-3^{n} \eta^{-n} \delta$. 
Lemma 4.4. Assume (4.6), $\mathbf{b}$ is harmonic function on $B_{16 r}(p)$ satisfying $\sup _{B_{16 r}(p)}|\nabla \mathbf{b}| \leq$ 2 and $f_{B_{16 r}(p)}|\nabla \mathbf{b}-\nabla \rho| \leq \delta$. Then we have

$$
\begin{array}{ll}
\frac{V\left(Q_{\eta, \mathbf{b}}^{r, \rho}\right)}{V\left(B_{r}(p)\right)} \geq 1-\left(12 \eta^{-1}\right)^{n} \delta & \text { and } \quad \frac{V\left(T_{\eta, \mathbf{b}}^{r, \rho}\right)}{V\left(B_{r}(p)\right)} \geq 1-n^{20 n} \eta^{-n} \sqrt{\delta}, \\
\frac{V\left(T_{\eta, \mathbf{b}}^{r, \rho}(x)\right)}{V\left(B_{r}(p)\right)} \geq 1-\sqrt{\eta}, & \forall x \in T_{\eta, \mathbf{b}}^{r, \rho} .
\end{array}
$$

Proof: From the assumption and Bishop-Gromov Comparison Theorem,

$$
f_{B_{4 r}}|\nabla \mathbf{b}-\nabla \rho| \leq \frac{V\left(B_{16 r}\right)}{V\left(B_{4 r}\right)} f_{B_{16 r}}|\nabla \mathbf{b}-\nabla \rho| \leq 4^{n} \delta .
$$

From the above inequality, apply Lemma 4.3 to $|\nabla \mathbf{b}-\nabla \rho|$, we get the first inequality of the conclusion. To prove the 3 rd inequality of the conclusion, we note

$$
\int_{B_{r} \backslash T_{\eta, \mathbf{b}}^{r, \rho}(x)} d y \int_{0}^{\hat{\rho}(x)-\eta r}\left(\int_{\gamma_{\sigma_{x}(s), \tilde{\sigma} y(s)}}\left|\nabla^{2} \mathbf{b}\right|\right) d s \geq \sqrt{\eta} r V\left(B_{r} \backslash T_{\eta, \mathbf{b}}^{r, \rho}(x)\right) .
$$

On the other hand, note $x \in T_{\eta, \mathbf{b}}^{r, \rho}$, we have

$$
\begin{aligned}
\int_{B_{r} \backslash T_{\eta, \mathbf{b}}^{r, \rho}(x)} d y \int_{0}^{\hat{\rho}(x)-\eta r}\left(\int_{\gamma_{\sigma_{x}(s), \tilde{\sigma} y(s)}}\left|\nabla^{2} \mathbf{b}\right|\right) d s & \leq V\left(B_{r}\right) f_{B_{r}} d y \int_{0}^{\hat{\rho}(x)-\eta r}\left(\int_{\gamma_{\sigma_{x}(s), \tilde{\sigma} y(s)}}\left|\nabla^{2} \mathbf{b}\right|\right) d s \\
& \leq V\left(B_{r}\right) \cdot \eta r .
\end{aligned}
$$

Hence we obtain $\frac{V\left(T_{\eta, \mathbf{b}}^{r, p}(x)\right)}{V\left(B_{r}\right)} \geq 1-\sqrt{\eta}$. Finally we prove the 2 nd inequality. From assumption and Lemma 2.4 , we get

$$
\begin{aligned}
f_{B_{8 r}}\left|\nabla^{2} \mathbf{b}\right| & \leq \frac{3 \cdot 10^{8} n^{5}(16 r)^{-1}}{2^{-\frac{3 n}{2}} \cdot 2^{-4}} \cdot\left(f_{B_{16 r}}|| \nabla \mathbf{b}|-1|\right)^{\frac{1}{2}} \\
& \leq n^{14 n} r^{-1}\left(f_{B_{16 r}}|\nabla \mathbf{b}-\nabla \rho|\right)^{\frac{1}{2}} \leq n^{14 n} r^{-1} \sqrt{\delta}
\end{aligned}
$$

Note we have

$$
\int_{B_{r} \backslash T_{\eta, \mathbf{b}}^{r, \rho}} d x f_{B_{r}} d y \int_{0}^{\hat{\rho}(x)-\eta r}\left(\int_{\gamma_{\sigma_{x}(s), \tilde{\sigma} y(s)}}\left|\nabla^{2} \mathbf{b}\right|\right) d s \geq \eta r \cdot V\left(B_{r} \backslash T_{\eta, \mathbf{b}}^{r, \rho}\right) .
$$


On the other hand, from Lemma 4.1, we have

$$
\begin{aligned}
& \int_{B_{r} \backslash T_{\eta, \mathbf{b}}^{r, \rho}} d x f_{B_{r}} d y \int_{0}^{\hat{\rho}(x)-\eta r}\left(\int_{\gamma_{\sigma_{x}(s), \tilde{\sigma} y(s)}}\left|\nabla^{2} \mathbf{b}\right|\right) d s \\
& \leq \frac{1}{V\left(B_{r}\right)} \int_{B_{r} \times B_{r}} d x d y \int_{0}^{\hat{\rho}(x)-\eta r}\left(\int_{\gamma_{\sigma_{x}(s), \tilde{\sigma} y}(s)}\left|\nabla^{2} \mathbf{b}\right|\right) d s \\
& \leq \frac{1}{V\left(B_{r}\right)} \int_{r}^{3 r} d t_{1} \int_{r}^{3 r} d t_{2} \int_{\left(\hat{\rho}^{-1}\left(t_{1}\right) \cap B_{r}\right) \times\left(\hat{\rho}^{-1}\left(t_{2}\right) \cap B_{r}\right)} d x d y \int_{0}^{t_{1}-\eta r}\left(\int_{\gamma_{\sigma_{x}(s), \tilde{\sigma}_{y}(s)}}\left|\nabla^{2} \mathbf{b}\right|\right) d s
\end{aligned}
$$

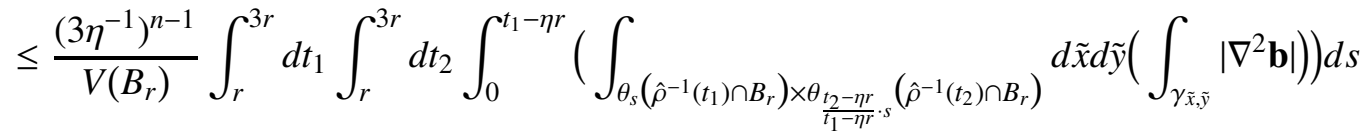

$$
\begin{aligned}
& \leq \frac{\left(3 \eta^{-1}\right)^{n-1}}{V\left(B_{r}\right)} \int_{r}^{3 r} d t_{1} \int_{0}^{t_{1}-\eta r} d s \int_{\theta_{s}\left(\hat{\rho}^{-1}\left(t_{1}\right) \cap B_{r}\right) \times B_{4 r}} d \tilde{x} d \tilde{y}\left(\int_{\gamma_{\tilde{x} \tilde{y}}}\left|\nabla^{2} \mathbf{b}\right|\right) \\
& \leq \frac{2\left(3 \eta^{-1}\right)^{n-1} r}{V\left(B_{r}\right)} \int_{B_{4 r} \times B_{4 r}} d \tilde{x} d \tilde{y}\left(\int_{\gamma_{\tilde{x}, \tilde{y}}}\left|\nabla^{2} \mathbf{b}\right|\right) \\
& \leq \frac{2\left(3 \eta^{-1}\right)^{n-1} r}{V\left(B_{r}\right)} 2^{n+1} \cdot(4 r) \cdot V\left(B_{4 r}\right) \int_{B_{8 r}}\left|\nabla^{2} \mathbf{b}\right| \\
& \leq 2^{6 n+4}\left(3 \eta^{-1}\right)^{n-1} r^{2} \cdot V\left(B_{r}\right) f_{B_{8 r}}\left|\nabla^{2} \mathbf{b}\right| \\
& \leq n^{20 n} \eta^{1-n} r \sqrt{\delta} \cdot V\left(B_{r}\right) \text {. }
\end{aligned}
$$

We used (4.7) in the last inequality above.

From above, we get

$$
\frac{V\left(T_{\eta, \mathbf{b}}^{r, \rho}\right)}{V\left(B_{r}\right)} \geq 1-n^{20 n} \eta^{-n} \sqrt{\delta} .
$$

For $x, y \in B_{r}(p)$, for each $\mathbf{b}_{i}$ from Theorem 2.13 and the corresponding $\rho_{i}, \mathfrak{P}_{i}$, we define

$$
\sigma_{x, i}(s)=\gamma_{\mathfrak{P}_{i}(x), x}(s+\eta r), \quad i=1, \cdots, k .
$$

Definition 4.5. For $0<\eta<\frac{1}{2} c_{1}$, we define

$$
\begin{aligned}
& Q_{\eta, \mathbf{b}_{i}}^{r, \rho_{i}, \rho_{j}}=\left\{x \in B_{r}(p): \int_{0}^{\hat{\rho}_{j}(x)-\eta r}\left|\nabla\left(\rho_{i}-\mathbf{b}_{i}\right)\right|\left(\sigma_{x, j}(s)\right) d s \leq \eta r\right\} \\
& P_{\eta, \mathbf{b}_{k}, \mathbf{b}_{l}}^{r, \rho_{j}}=\left\{x \in B_{r}(p): \int_{0}^{\hat{\rho}_{j}(x)-\eta r}\left|\left\langle\nabla \mathbf{b}_{k}, \nabla \mathbf{b}_{l}\right\rangle\right|\left(\sigma_{x, j}(s)\right) d s \leq \eta r\right\} .
\end{aligned}
$$

Lemma 4.6. Assume $0<r \leq \hat{\rho}_{j}(x) \leq 3 r$ for any $x \in B_{r}(p)$, if

$$
f_{B_{4 r}(p)}\left|\left\langle\nabla \mathbf{b}_{k}, \nabla \mathbf{b}_{l}\right\rangle\right| \leq \delta \quad \text { and } \quad f_{B_{4 r}(p)}\left|\nabla\left(\mathbf{b}_{i}-\rho_{i}\right)\right| \leq \delta \text {, }
$$


then we have

$$
\frac{V\left(Q_{\eta, \mathbf{b}_{i}}^{r, \rho_{i}, \rho_{j}}\right)}{V\left(B_{r}(p)\right)} \geq 1-\left(3 \eta^{-1}\right)^{n} \delta \quad \text { and } \quad \frac{V\left(P_{\eta, \mathbf{b}_{k}, \mathbf{b}_{l}}^{r, \rho_{l}}\right)}{V\left(B_{r}(p)\right)} \geq 1-\left(3 \eta^{-1}\right)^{n} \delta .
$$

Proof: Apply Lemma 4.3 to $\left|\nabla\left(\mathbf{b}_{i}-\rho_{i}\right)\right|$ and $\left|\left\langle\nabla \mathbf{b}_{k}, \nabla \mathbf{b}_{l}\right\rangle\right|$ respectively, we get our conclusion.

\section{Quantitative almost SPLITTING THEOREM}

The main results of this section were sort of implied in Cheeger-Colding's work (see [CC96] and [CC97]), however we will not follow their argument there. Instead, we adapt the argument of Colding-Naber in [CN12] to prove the main result of this section. Although our argument has close relationship with [CC96] and [CC97], the main difference is that the angle between two segments is not involved into our argument, and the first variation formula is applied to the case of both end points are moving.

Lemma 5.1. For any $0<\eta<\frac{1}{2}$, assume (4.6), $\mathbf{b}$ is harmonic function on $B_{16 r}(p)$ satisfying $\sup _{B_{r}(p)}|\nabla \mathbf{b}| \leq 2$ and $\sup _{B_{r}(p)}|\mathbf{b}-\rho| \leq \eta r$. Then for any $x \in T_{\eta, \mathbf{b}}^{r, \rho} \cap Q_{\eta, \mathbf{b}}^{r, \rho}$, $y \in T_{\eta, \mathbf{b}}^{r, \rho}(x) \cap Q_{\eta, \mathbf{b}}^{r, \rho}$, we have $\left|d(x, y)-d\left(\mathfrak{5}_{\rho}(x), \mathfrak{5}_{\rho}(y)\right)\right| \leq 5000 \eta^{\frac{1}{8}} \cdot r$.

Proof: Using the first variation formula for arc length, and note

$$
\begin{gathered}
\sigma_{x}^{\prime}=\nabla \rho, \quad \text { and } \quad \tilde{\sigma}_{y}^{\prime}=\frac{\hat{\rho}(y)-\eta r}{\hat{\rho}(x)-\eta r} \cdot \nabla \rho, \\
l_{t}-l_{0}=d\left(\sigma_{x}(t), \tilde{\sigma}_{y}(t)\right)-d\left(\sigma_{x}(0), \tilde{\sigma}_{y}(0)\right) \\
=\int_{0}^{t}\left\langle\tilde{\sigma}_{y}^{\prime}, \tau_{s}^{\prime}\right\rangle\left(\tau_{s}\left(l_{s}\right)\right)-\left\langle\sigma_{x}^{\prime}, \tau_{s}^{\prime}\right\rangle\left(\tau_{s}(0)\right) \\
=\int_{0}^{t}\left\langle\tilde{\sigma}_{y}^{\prime}-\nabla \mathbf{b}, \tau_{s}^{\prime}\right\rangle\left(\tau_{s}\left(l_{s}\right)\right)-\left\langle\sigma_{x}^{\prime}-\nabla \mathbf{b}, \tau_{s}^{\prime}\right\rangle\left(\tau_{s}(0)\right) \\
+\int_{0}^{t}\left\langle\nabla \mathbf{b}, \tau_{s}^{\prime}\right\rangle\left(\tau_{s}\left(l_{s}\right)\right)-\left\langle\nabla \mathbf{b}, \tau_{s}^{\prime}\right\rangle\left(\tau_{s}(0)\right) d s \\
=\int_{0}^{t}\left\langle\frac{\hat{\rho}(y)-\eta r}{\hat{\rho}(x)-\eta r} \nabla \rho-\nabla \mathbf{b}, \tau_{s}^{\prime}\right\rangle\left(\tau_{s}\left(l_{s}\right)\right)-\left\langle\nabla(\rho-\mathbf{b}), \tau_{s}^{\prime}\right\rangle\left(\tau_{s}(0)\right) \\
+\int_{0}^{t} \int_{0}^{l_{s}} \nabla \nabla^{2} \mathbf{b}\left(\tau_{s}^{\prime}, \tau_{s}^{\prime}\right)\left(\tau_{s}(v)\right) d v d s .
\end{gathered}
$$

Step (1). If

$$
l_{0} \leq 40 \eta^{\frac{1}{8}} r
$$


From (5.1) and $x, y \in Q_{\eta, \mathbf{b}}^{r, \rho}$, we have

$$
\begin{aligned}
& d(x, y)-l_{0}=l_{\hat{\rho}(x)-\eta r}-l_{0} \\
\leq & \left|\frac{\hat{\rho}(y)-\hat{\rho}(x)}{\hat{\rho}(x)-\eta r}\right| \cdot \int_{0}^{\hat{\rho}(x)-\eta r}\left\langle\nabla \rho, \tau_{s}^{\prime}\right\rangle\left(\tau_{s}\left(l_{s}\right)\right) d s \\
& +\int_{0}^{\hat{\rho}(x)-\eta r}|\nabla(\rho-\mathbf{b})|\left(\tilde{\sigma}_{y}(s)\right)+|\nabla(\rho-\mathbf{b})|\left(\sigma_{x}(s)\right) d s \\
& +\int_{0}^{\hat{\rho}(x)-\eta r} \int_{0}^{l_{s}}\left|\nabla^{2} \mathbf{b}\right|\left(\tau_{s}(v)\right) d v d s \\
\leq & |\hat{\rho}(y)-\hat{\rho}(x)|+\frac{\hat{\rho}(x)-\eta r}{\hat{\rho}(y)-\eta r} \int_{0}^{\hat{\rho}(y)-\eta r}|\nabla \mathbf{b}-\nabla \rho|\left(\sigma_{y}(s)\right) d s \\
& +\int_{0}^{\hat{\rho}(x)-\eta r}|\nabla \mathbf{b}-\nabla \rho|\left(\sigma_{x}(s)\right) d s+\sqrt{\eta} r \\
\leq & |\hat{\rho}(y)-\hat{\rho}(x)|+8 \sqrt{\eta} r .
\end{aligned}
$$

Hence

$$
d(x, y)-|\hat{\rho}(y)-\hat{\rho}(x)| \leq l_{0}+8 \sqrt{\eta} r .
$$

Note $d(x, y) \geq|\hat{\rho}(y)-\hat{\rho}(x)|$, by $d\left(\mathfrak{F}_{\rho}(x), \mathfrak{b}_{\rho}(y)\right)=\sqrt{d(\mathfrak{P}(x), \mathfrak{P}(y))^{2}+|\hat{\rho}(y)-\hat{\rho}(x)|^{2}}$, (5.2) and (5.3), we have

$$
\begin{aligned}
& \left|d(x, y)-d\left(\mathfrak{b}_{\rho}(x), \mathfrak{G}_{\rho}(y)\right)\right| \\
\leq & |d(x, y)-| \hat{\rho}(y)-\hat{\rho}(x)|+||\hat{\rho}(y)-\hat{\rho}(x)|-d\left(\mathfrak{b}_{\rho}(x), \mathfrak{b}_{\rho}(y)\right) \mid \\
\leq & d(x, y)-|\hat{\rho}(y)-\hat{\rho}(x)|+d(\mathfrak{P}(x), \mathfrak{P}(y)) \\
\leq & l_{0}+8 \sqrt{\eta} r+l_{0}+2 \sqrt{\eta} r \leq 90 \eta^{\frac{1}{8}} r .
\end{aligned}
$$

Step (2). In the rest of the proof, we assume that

$$
l_{0}>40 \eta^{\frac{1}{8}} r .
$$

From (5.1) and $x, y \in Q_{\eta, \mathbf{b}}^{r, \rho}$, for $t \in[0, \hat{\rho}(x)-\eta r]$, we get

$$
\begin{aligned}
& \left|l_{t}-l_{0}-\left(\frac{\hat{\rho}(y)-\hat{\rho}(x)}{\hat{\rho}(x)-\eta r}\right) \cdot \int_{0}^{t}\left\langle\nabla \mathbf{b}, \tau_{s}^{\prime}\right\rangle\left(\tau_{s}\left(l_{s}\right)\right)\right| \\
\leq & \int_{0}^{t}|\nabla(\rho-\mathbf{b})|\left(\sigma_{x}(s)\right)+\frac{\hat{\rho}(y)-\eta r}{\hat{\rho}(x)-\eta r}|\nabla(\rho-\mathbf{b})|\left(\tilde{\sigma}_{y}(s)\right) \\
& +\int_{0}^{t} \int_{0}^{l_{s}}\left|\nabla^{2} \mathbf{b}\right|\left(\tau_{s}(v)\right) d v d s \\
\leq & \int_{0}^{t}|\nabla \mathbf{b}-\nabla \rho|\left(\sigma_{x}(s)\right)+\int_{0}^{\frac{\hat{\rho}(y)-\eta r}{\hat{\rho}(x)-\eta r} t}|\nabla \mathbf{b}-\nabla \rho|\left(\sigma_{y}(s)\right) d s+\sqrt{\eta} r \\
\leq & 3 \sqrt{\eta} r .
\end{aligned}
$$


Now we estimate $\left\langle\nabla \mathbf{b}, \tau_{s}^{\prime}\right\rangle\left(\tau_{s}\left(l_{s}\right)\right)$. For any $0 \leq t_{1} \leq l_{s}$, we have

$$
\left(\mathbf{b} \circ \tau_{s}\right)^{\prime}\left(l_{s}\right) \leq\left(\mathbf{b} \circ \tau_{s}\right)^{\prime}\left(t_{1}\right)+\int_{0}^{l_{s}}\left|\left(\mathbf{b} \circ \tau_{s}\right)^{\prime \prime}(t)\right| d t,
$$

Take the integral of the above inequality from 0 to $l_{s}$ with respect to $t_{1}$, we get

$$
\begin{aligned}
l_{s} \cdot\left(\mathbf{b} \circ \tau_{s}\right)^{\prime}\left(l_{s}\right) & \leq\left[\left(\mathbf{b} \circ \tau_{s}\right)\left(l_{s}\right)-\left(\mathbf{b} \circ \tau_{s}\right)(0)\right]+l_{s} \cdot \int_{0}^{l_{s}}\left|\nabla^{2} \mathbf{b}\right|\left(\tau_{s}(t)\right) d t \\
& \leq 2 \eta r+\left[\left(\rho \circ \tau_{s}\right)\left(l_{s}\right)-\left(\rho \circ \tau_{s}\right)(0)\right]+l_{s} \cdot \int_{0}^{l_{s}}\left|\nabla^{2} \mathbf{b}\right|\left(\tau_{s}(t)\right) d t \\
& =2 \eta r+\left(\frac{\hat{\rho}(y)-\hat{\rho}(x)}{\hat{\rho}(x)-\eta r}\right) \cdot s+l_{s} \cdot \int_{0}^{l_{s}}\left|\nabla^{2} \mathbf{b}\right|\left(\tau_{s}(t)\right) d t .
\end{aligned}
$$

In the second inequality above we used the assumption $\sup _{B_{r}(p)}|\mathbf{b}-\rho| \leq \eta r$. Then

$$
\left(\mathbf{b} \circ \tau_{s}\right)^{\prime}\left(l_{s}\right) \leq \frac{s}{l_{s}} \cdot\left(\frac{\hat{\rho}(y)-\hat{\rho}(x)}{\hat{\rho}(x)-\eta r}\right)+\frac{2 \eta r}{l_{s}}+\int_{0}^{l_{s}}\left|\nabla^{2} \mathbf{b}\right|\left(\tau_{s}(t)\right) d t .
$$

Similarly, we can also have

$$
\left(\mathbf{b} \circ \tau_{s}\right)^{\prime}\left(l_{s}\right) \geq \frac{s}{l_{s}} \cdot\left(\frac{\hat{\rho}(y)-\hat{\rho}(x)}{\hat{\rho}(x)-\eta r}\right)-\frac{2 \eta r}{l_{s}}-\int_{0}^{l_{s}}\left|\nabla^{2} \mathbf{b}\right|\left(\tau_{s}(t)\right) d t .
$$

Hence we have

$$
\left|\left(\mathbf{b} \circ \tau_{s}\right)^{\prime}\left(l_{s}\right)-\frac{s}{l_{s}} \cdot\left(\frac{\hat{\rho}(y)-\hat{\rho}(x)}{\hat{\rho}(x)-\eta r}\right)\right| \leq \frac{2 \eta r}{l_{s}}+\int_{0}^{l_{s}}\left|\nabla^{2} \mathbf{b}\right|\left(\tau_{s}(t)\right) d t .
$$

Step (3). We will show the uniform lower bound of $l_{t}$ when $t \in[0, \hat{\rho}(x)-\eta r]$. There are two cases to be discussed.

(3.A) If $|\hat{\rho}(y)-\hat{\rho}(x)| \leq \frac{1}{4} l_{0}$. From (5.5) and $|\nabla \mathbf{b}| \leq 2$,

$$
\begin{aligned}
l_{t}-l_{0} & \geq\left(\frac{\hat{\rho}(y)-\hat{\rho}(x)}{\hat{\rho}(x)-\eta r}\right) \cdot \int_{0}^{t}\left\langle\nabla \mathbf{b}, \tau_{s}^{\prime}\right\rangle\left(\tau_{s}\left(l_{s}\right)\right) d s-3 \sqrt{\eta} r \\
& \geq-2|\hat{\rho}(y)-\hat{\rho}(x)|-3 \sqrt{\eta} r \geq-\frac{1}{2} l_{0}-3 \sqrt{\eta} r .
\end{aligned}
$$

From (5.4), we get

$$
l_{t} \geq \frac{1}{2} l_{0}-3 \sqrt{\eta} r \geq \eta^{\frac{1}{4}} r .
$$

(3.B) If $|\hat{\rho}(y)-\hat{\rho}(x)|>\frac{1}{4} l_{0}$. Let $\left(\frac{\hat{\rho}(y)-\hat{\rho}(x)}{\hat{\rho}(x)-\eta r}\right)^{2}=\alpha_{1}$, from (5.6), (5.7) and (5.5), we can get

$$
\begin{aligned}
l_{t}-l_{0} & \geq\left(\frac{\hat{\rho}(y)-\hat{\rho}(x)}{\hat{\rho}(x)-\eta r}\right) \cdot \int_{0}^{t}\left\langle\nabla \mathbf{b}, \tau_{s}^{\prime}\right\rangle\left(\tau_{s}\left(l_{s}\right)\right) d s-3 \sqrt{\eta} r \\
& \geq \alpha_{1} \int_{0}^{t} \frac{s}{l_{s}} d s-\sqrt{\alpha_{1}} \cdot \int_{0}^{t}\left(\frac{2 \eta r}{l_{s}}+\int_{0}^{l_{s}}\left|\nabla^{2} \mathbf{b}\right|\left(\tau_{s}(t)\right) d t\right) d s-3 \sqrt{\eta} r \\
\quad & \geq-\sqrt{\alpha_{1}} \cdot 2 \eta r \int_{0}^{\frac{2 \eta r}{\sqrt{\alpha_{1}}}} \frac{1}{l_{s}} d s-16 \int_{0}^{\hat{\rho}(x)-\eta r} \int_{0}^{l_{s}}\left|\nabla^{2} \mathbf{b}\right|\left(\tau_{s}(t)\right) d t d s-3 \sqrt{\eta} r .
\end{aligned}
$$


Note for any $0 \leq s \leq \frac{2 \eta r}{\sqrt{\alpha_{1}}}$, using $\sqrt{\alpha_{1}} \geq 2^{-2} \cdot \frac{l_{0}}{\hat{\rho}(x)-\eta r} \geq \frac{l_{0}}{12 r}$ and (5.4), we have

$$
\begin{aligned}
l_{s} & \geq l_{0}-s-\frac{\hat{\rho}(y)-\eta r}{\hat{\rho}(x)-\eta r} s \geq l_{0}-\left(1+\frac{3 r-\eta r}{r-\eta r}\right) \frac{2 \eta r}{\sqrt{\alpha_{1}}} \geq l_{0}-7 \cdot 2 \eta r \cdot \frac{12 r}{l_{0}} \\
& \geq l_{0}-\frac{200 \eta r^{2}}{l_{0}} \geq \sqrt{\eta} r .
\end{aligned}
$$

From (5.9) and (5.10), we get

$$
l_{t}-l_{0} \geq-\sqrt{\alpha_{1}} \cdot 2 \eta r \cdot \frac{2 \eta r}{\sqrt{\alpha_{1}}} \cdot \frac{1}{\sqrt{\eta} r}-19 \sqrt{\eta} r \geq-25 \sqrt{\eta} r .
$$

Then by (5.4) again,

$$
l_{t} \geq l_{0}-25 \sqrt{\eta} r \geq \eta^{\frac{1}{4}} r .
$$

From above two cases, we always have

$$
l_{t} \geq \eta^{\frac{1}{4}} r, \quad \forall t \in[0, \hat{\rho}(x)-\eta r] .
$$

Step (4). From (5.8), (5.11) and $\eta \geq \delta$, we obtain

$$
\begin{aligned}
& \left|\left(\frac{\hat{\rho}(y)-\hat{\rho}(x)}{\hat{\rho}(x)-\eta r}\right) \cdot \int_{0}^{t}\left\langle\nabla \mathbf{b}, \tau_{s}^{\prime}\right\rangle\left(\tau_{s}\left(l_{s}\right)\right)-\alpha_{1} \int_{0}^{t} \frac{s}{l_{s}} d s\right| \\
\leq & \left|\frac{\hat{\rho}(y)-\hat{\rho}(x)}{\hat{\rho}(x)-\eta r}\right| \int_{0}^{t}\left(\frac{2 \eta r}{l_{s}}+\int_{0}^{l_{s}}\left|\nabla^{2} \mathbf{b}\right|\left(\tau_{s}(t)\right) d t\right) d s \\
\leq & 3 r \cdot \frac{8 \eta}{\eta^{\frac{1}{4}}}+4 \int_{0}^{\hat{\rho}(x)-\eta r} \int_{0}^{l_{s}}\left|\nabla^{2} \mathbf{b}\right|\left(\tau_{s}(t)\right) d t d s \leq 28 \sqrt{\eta} r .
\end{aligned}
$$

From (5.5) and (5.12), we get

$$
\left|l_{t}-l_{0}-\alpha_{1} \int_{0}^{t} \frac{s}{l_{s}} d s\right| \leq 31 \sqrt{\eta} r .
$$

Define $\mathfrak{L}(t)=\int_{0}^{t} \frac{s}{l_{s}} d s$, then $l_{t}=\frac{t}{\mathfrak{I}^{\prime}(t)}$ and we have

$$
\left|\frac{t}{\mathfrak{L}^{\prime}(t)}-\alpha_{1} \mathfrak{L}(t)-l_{0}\right| \leq 31 \sqrt{\eta} r .
$$

On the other hand, let $f(s)=\sqrt{\alpha_{1} s^{2}+l_{0}^{2}}$, and define

$$
F(t)=\int_{0}^{t} \frac{s}{f(s)} d s
$$

Then it is easy to get

$$
\frac{t}{F(t)^{\prime}}-\alpha_{1} F(t)-l_{0}=0
$$


From (5.13) and (5.14), we obtain

$$
\begin{aligned}
\left|\left[\left(\frac{1}{2} \alpha_{1} \mathfrak{L}^{2}+l_{0} \mathfrak{L}\right)-\left(\frac{1}{2} \alpha_{1} F^{2}+l_{0} F\right)\right]^{\prime}\right| & \leq\left|31 \sqrt{\eta} r \cdot \mathfrak{L}^{\prime}\right|=\left|31 \sqrt{\eta} r \cdot \frac{t}{l_{t}}\right| \\
& \leq\left|31 \sqrt{\eta} r \cdot \frac{3 r}{\eta^{\frac{1}{4}} r}\right| \leq 100 \eta^{\frac{1}{4}} r .
\end{aligned}
$$

Take the integral of the above inequality, also note $\mathfrak{L}(0)=F(0)=0$ and $0 \leq t \leq$ $\hat{\rho}(x)-\eta r \leq 3 r$, we have

$$
\left|\left(\frac{1}{2} \alpha_{1} \mathfrak{L}(t)^{2}+l_{0} \mathfrak{Q}(t)\right)-\left(\frac{1}{2} \alpha_{1} F(t)^{2}+l_{0} F(t)\right)\right| \leq 300 \eta^{\frac{1}{4}} r^{2} .
$$

Simplify the above inequality, note $\mathfrak{L}(t) \geq 0$ and $F(t) \geq 0$, using $l_{0} \geq \eta^{\frac{1}{8}} r$,

$$
\begin{aligned}
|\mathfrak{L}(t)-F(t)| & \leq l_{0}^{-1}\left|\left(\frac{1}{2} \alpha_{1} \mathfrak{Q}(t)^{2}+l_{0} \mathfrak{Q}(t)\right)-\left(\frac{1}{2} \alpha_{1} F(t)^{2}+l_{0} F(t)\right)\right| \\
& \leq 300 \eta^{\frac{1}{8}} r .
\end{aligned}
$$

From (5.13), (5.14) and (5.15), we have

$$
\left|l_{t}-f(t)\right|=\left|\frac{t}{\mathfrak{L}^{\prime}(t)}-\frac{t}{F(t)^{\prime}}\right| \leq \alpha_{1} \cdot 300 \eta^{\frac{1}{8}} r+31 \sqrt{\eta} r \leq 4831 \eta^{\frac{1}{8}} r .
$$

Let $t=\hat{\rho}(x)-\eta r$ in $(5.16)$, note $\left|l_{0}-d(\mathfrak{P}(x), \mathfrak{P}(y))\right| \leq 2 \eta r$ and $l_{\hat{\rho}(x)-\eta r}=d(x, y)$,

$$
\begin{aligned}
& \left|d(x, y)-d\left(\mathfrak{b}_{\rho}(x), \mathfrak{b}_{\rho}(y)\right)\right| \\
\leq & \left|f(\hat{\rho}(x)-\eta r)-l_{\hat{\rho}(x)-\eta r}\right|+\left|d\left(\mathfrak{b}_{\rho}(x), \mathfrak{b}_{\rho}(y)\right)-f(\hat{\rho}(x)-\eta r)\right| \\
\leq & 4831 \eta^{\frac{1}{8}} r+\left|\sqrt{d(\mathfrak{P}(x), \mathfrak{P}(y))^{2}+(\hat{\rho}(y)-\hat{\rho}(x))^{2}}-\sqrt{l_{0}^{2}+(\hat{\rho}(y)-\hat{\rho}(x))^{2}}\right| \\
\leq & 4831 \eta^{\frac{1}{8}} r+2 \eta r \leq 5000 \eta^{\frac{1}{8}} r .
\end{aligned}
$$

Corollary 5.2. For any $\eta \in(0,1)$, assume (4.6) and

$$
\begin{aligned}
& \sup _{B_{16 r}(p)}|\nabla \mathbf{b}| \leq 2, \quad \sup _{B_{r}(p)}|\mathbf{b}-\rho| \leq \eta r \\
& f_{B_{16 r}(p)}|\nabla \mathbf{b}-\nabla \rho| \leq 2^{-n} \eta^{3 n},
\end{aligned}
$$

then there exists $\delta_{1}=n^{21} \eta^{\frac{1}{2 n}}$ such that $\sup _{x, y \in T_{\eta, \mathbf{b}}^{r, \rho} \cap Q_{\eta, \mathbf{b}}^{r, \rho} \cap B_{\left(1-\delta_{1}\right) r}(p)}\left|d(x, y)-d\left(\mathfrak{5}_{\rho}(x), \mathfrak{5}_{\rho}(y)\right)\right| \leq$ $n^{22} \eta^{\frac{1}{3 n}} r$ 
Proof: From Lemma 4.4, for any $x, y \in T_{\eta, \mathbf{b}}^{r, \rho} \cap Q_{\eta, \mathbf{b}}^{r, \rho} \cap B_{\left(1-\delta_{1}\right) r}(p)$, we have

$$
\begin{aligned}
& V\left(B_{r}(p) \backslash\left(T_{\eta, \mathbf{b}}^{r, \rho} \cap Q_{\eta, \mathbf{b}}^{r, \rho} \cap T_{\eta, \mathbf{b}}^{r, \rho}(x) \cap T_{\eta, \mathbf{b}}^{r, \rho}(y)\right)\right) \\
= & V\left(\left(B_{r}(p) \backslash T_{\eta, \mathbf{b}}^{r, \rho}\right) \cup\left(B_{r}(p) \backslash Q_{\eta, \mathbf{b}}^{r, \rho}\right) \cup\left(B_{r}(p) \backslash T_{\eta, \mathbf{b}}^{r, \rho}(x)\right) \cup\left(B_{r}(p) \backslash T_{\eta, \mathbf{b}}^{r, \rho}(y)\right)\right) \\
\leq & V\left(B_{r}(p) \backslash T_{\eta, \mathbf{b}}^{r, \rho}\right)+V\left(B_{r}(p) \backslash Q_{\eta, \mathbf{b}}^{r, \rho}\right)+V\left(B_{r}(p) \backslash T_{\eta, \mathbf{b}}^{r, \rho}(x)\right)+V\left(B_{r}(p) \backslash T_{\eta, \mathbf{b}}^{r, \rho}(y)\right) \\
\leq & 2\left(n^{20 n} \eta^{-n} \sqrt{2^{-n} \eta^{3 n}}+\sqrt{\eta}\right) V\left(B_{r}(p)\right) \leq n^{20 n} \eta^{\frac{1}{2}} V\left(B_{r}(p)\right) .
\end{aligned}
$$

We claim that $T_{\eta, \mathbf{b}}^{r, \rho} \cap Q_{\eta, \mathbf{b}}^{r, \rho} \cap T_{\eta, \mathbf{b}}^{r, \rho}(x) \cap T_{\eta, \mathbf{b}}^{r, \rho}(y) \cap B_{\delta_{1} r}(x) \neq \emptyset$, otherwise

$$
B_{\delta_{1} r}(x) \subset B_{r}(p) \backslash\left(T_{\eta, \mathbf{b}}^{r, \rho} \cap Q_{\eta, \mathbf{b}}^{r, \rho} \cap T_{\eta, \mathbf{b}}^{r, \rho}(x) \cap T_{\eta, \mathbf{b}}^{r, \rho}(y)\right),
$$

which implies

$$
\frac{V\left(B_{\delta_{1} r}(x)\right)}{V\left(B_{r}(p)\right)} \leq \frac{V\left(B_{r}(p) \backslash\left(T_{\eta, \mathbf{b}}^{r, \rho} \cap Q_{\eta, \mathbf{b}}^{r, \rho} \cap T_{\eta, \mathbf{b}}^{r, \rho}(x) \cap T_{\eta, \mathbf{b}}^{r, \rho}(y)\right)\right)}{V\left(B_{r}(p)\right)} \leq n^{20 n} \eta^{\frac{1}{2}} .
$$

On the other hand, from Bishop-Gromov Comparison Theorem and $B_{r}(p) \subset B_{2 r}(x)$, using the definition of $\delta_{1}$, we have

$$
\frac{V\left(B_{\delta_{1} r}(x)\right)}{V\left(B_{r}(p)\right)} \geq \frac{V\left(B_{\delta_{1} r}(x)\right)}{V\left(B_{2 r}(x)\right)} \geq\left(\frac{\delta_{1}}{2}\right)^{n}>n^{20 n} \eta^{\frac{1}{2}}
$$

which is the contradiction. Hence we can find

$$
z \in T_{\eta, \mathbf{b}}^{r, \rho} \cap Q_{\eta, \mathbf{b}}^{r, \rho} \cap T_{\eta, \mathbf{b}}^{r, \rho}(x) \cap T_{\eta, \mathbf{b}}^{r, \rho}(y) \cap B_{\delta_{1} r}(x) .
$$

Now apply Lemma 5.1 to $y, z$ and $x, z$ respectively, then we have

$$
\begin{aligned}
& \left|d(x, y)-d\left(\mathfrak{G}_{\rho}(x), \mathfrak{5}_{\rho}(y)\right)\right| \leq|d(x, y)-d(z, y)|+\left|d(z, y)-d\left(\mathfrak{b}_{\rho}(z), \mathfrak{G}_{\rho}(y)\right)\right| \\
& +\left|d\left(\mathfrak{G}_{\rho}(z), \mathfrak{b}_{\rho}(y)\right)-d\left(\mathfrak{b}_{\rho}(x), \mathfrak{b}_{\rho}(y)\right)\right| \\
& \leq d(x, z)+5000 \eta^{\frac{1}{8}} r+d\left(\mathfrak{b}_{\rho}(x), \mathfrak{b}_{\rho}(z)\right) \\
& \leq 10^{4} \eta^{\frac{1}{8}} r+2 d(x, z) \leq 10^{4} \eta^{\frac{1}{8}} r+2 \delta_{1} r \\
& \leq n^{22} \eta^{\frac{1}{3 n}} r \text {. }
\end{aligned}
$$

Proof: [of Theorem 2.13] Step (1). We firstly deal with the case $k=1$. From Proposition 3.4, there exists $\delta \leq 2^{-100 n^{2}} \epsilon_{1}^{2 n^{2}}$, where $\epsilon_{1}>0$ is a constant to be determined later, such that if (2.43) holds for $\delta$, we can find two functions $\rho_{1}, \tilde{\rho}_{1}$ 
satisfying the following

$$
\begin{aligned}
& \rho_{1}(x)=\hat{\rho}_{1}(x)+t_{0}=d\left(x, \rho^{-1}\left(t_{0}\right)\right)+t_{0}, \quad \tilde{\rho}_{1}(x)=t_{1}-d\left(x, \tilde{\rho}^{-1}\left(t_{1}\right)\right) \\
& \frac{r}{320} \leq \hat{\rho}_{1}(x) \leq \frac{3 r}{320}, \quad \forall x \in B_{\frac{r}{320}}(p) \\
& \sup _{B_{\frac{1}{20} r}(p)}\left|\mathbf{b}_{1}-\rho_{1}\right| \leq \epsilon_{1} \cdot r \quad \text { and } \quad f_{B_{\frac{1}{20} r}(p)}\left|\nabla\left(\mathbf{b}_{1}-\rho_{1}\right)\right| \leq \epsilon_{1} \\
& \rho_{1}(x) \leq \tilde{\rho}_{1}(x)+\frac{\epsilon_{1} r}{2}, \quad \forall x \in B_{\frac{r}{10}}(p) .
\end{aligned}
$$

From (5.19), (2.43) and (5.20), for $\eta \in(0,1)$ to be determined later, if we assume

$$
\epsilon_{1} \leq 2^{-n} \eta^{3 n}
$$

apply Corollary 5.2, there exist $\delta_{1}=n^{21} \eta^{\frac{1}{2 n}}$ such that

$$
\sup _{x, y \in T_{\eta, \mathbf{b}_{1}}^{\frac{r}{32}, \rho_{1}} \cap Q_{\eta, \mathbf{b}_{1}}^{\frac{r}{32,}, \rho_{1}} \cap B_{\frac{\left(1-\delta_{1}\right) r}{320}}(p)}\left|d(x, y)-d\left(\mathfrak{G}_{\rho_{1}}(x), \mathfrak{5}_{\rho_{1}}(y)\right)\right| \leq \frac{n^{22}}{320} \eta^{\frac{1}{3 n}} r .
$$

Now choose a maximal collection of disjoint balls with radius $\frac{\delta_{1} r}{320}$ centered at $B_{\frac{\left(1-\delta_{1}\right) r}{320}}(p)$, denoted as $\left\{B_{\frac{\delta_{1} r}{320}}\left(x_{i}\right)\right\}_{i=1}^{m}$, then

$$
B_{\frac{r}{320}}(p) \subset \bigcup_{i=1}^{m} B_{\frac{3 \delta_{1} r}{320}}\left(x_{i}\right) \quad \text { and } \quad \bigcup_{i=1}^{m} B_{\frac{\delta_{1} r}{320}}\left(x_{i}\right) \subset B_{\frac{r}{320}}(p) .
$$

From Lemma 4.4, (5.19), (5.22) and (5.20), we have

$$
\begin{aligned}
\frac{V\left(B_{\frac{r}{320}}(p) \backslash\left(T_{\eta, \mathbf{b}_{1}}^{\frac{r}{320}, \rho_{1}} \cap Q_{\eta, \mathbf{b}_{1}}^{\frac{r}{320}, \rho_{1}}\right)\right)}{V\left(B_{\frac{r}{320}}(p)\right)} & \leq \frac{V\left(B_{\frac{r}{320}}(p) \backslash T_{\eta, \mathbf{b}_{1}}^{\frac{r}{320}, \rho_{1}}\right)}{V\left(B_{\frac{r}{320}}(p)\right)}+\frac{V\left(B_{\frac{r}{320}}(p) \backslash Q_{\eta, \mathbf{b}_{1}}^{\frac{r}{320}, \rho_{1}}\right)}{V\left(B_{\frac{r}{320}}(p)\right)} \\
& \leq\left(12 \eta^{-1}\right)^{n} \epsilon_{1}+n^{20} \eta^{-n} \sqrt{\epsilon_{1}} \leq n^{20 n} \eta^{\frac{n}{2}} .
\end{aligned}
$$

On the other hand, from Bishop-Gromov Comparison Theorem and (5.24),

$$
\frac{V\left(B_{\frac{\delta_{1} r}{320}}\left(x_{i}\right)\right)}{V\left(B_{\frac{r}{320}}(p)\right)} \geq \frac{V\left(B_{\frac{\delta_{1}}{32}}\left(x_{i}\right)\right)}{V\left(B_{\frac{2 r}{320}}\left(x_{i}\right)\right)} \geq\left(\frac{\delta_{1}}{2}\right)^{n}>n^{20 n} \eta^{\frac{n}{2}} .
$$

From (5.25) and (5.26), we get $V\left(B_{\frac{\delta_{1} r}{320}}\left(x_{i}\right)\right)>V\left(B_{\frac{r}{320}}(p) \backslash\left(T_{\eta, \mathbf{b}_{1}}^{\frac{r}{320}, \rho_{1}} \cap Q_{\eta, \mathbf{b}_{1}}^{\frac{r}{320}, \rho_{1}}\right)\right)$. So there exists $y_{i}$ such that $y_{i} \in B_{\frac{\delta_{1} r}{320}}\left(x_{i}\right) \cap T_{\eta, \mathbf{b}_{1}}^{\frac{r}{320}, \rho_{1}} \cap Q_{\eta, \mathbf{b}_{1}}^{\frac{r}{320}, \rho_{1}}$, combining (5.24) yields

$$
B_{\frac{r}{320}}(p) \subset \bigcup_{i=1}^{m} B_{\frac{\delta_{1} r}{80}}\left(y_{i}\right) \text {. }
$$

Now we define $\mathbf{Y}=\left\{y_{1}, \cdots, y_{m}\right\} \cap B_{\frac{r}{1280}-n^{22} \eta^{\frac{1}{3 n}} r}(p)$ and $\mathbf{X}_{1}=\bigcup_{y_{i} \in \mathbf{Y}} \mathfrak{P}_{1}\left(y_{i}\right) \subset \mathbf{X}_{\mathbf{b}_{1}}$, which is a metric space with distance function $d$ inherited from the metric $g$ of the Riemannian manifold $M^{n}$. 
Step (2). For any $x \in B_{\frac{r}{1280}}(p)$, we define

$\mathfrak{g}_{1}(x)=\left(\hat{\rho}_{1}\left(\mathfrak{n}_{1}(x)\right)-\hat{\rho}_{1}\left(\mathfrak{n}_{1}(p)\right), \mathfrak{P}_{1}\left(\mathfrak{n}_{1}(x)\right)\right): B_{\frac{r}{1280}}(p) \rightarrow B_{\frac{r}{1280}}(0, \hat{p}) \subset \mathbb{R} \times \mathbf{X}_{1}$,

where $\hat{p}=\mathfrak{P}_{1}\left(\mathfrak{n}_{1}(p)\right)$ and

$$
\mathfrak{n}_{1}(x) \in \mathcal{D}_{x}:=\left\{y_{i} \in \mathbf{Y} \mid d\left(y_{i}, x\right)=\min _{y \in \mathbf{Y}} d(y, x)\right\} .
$$

If $\mathcal{D}_{x}$ contains more than one elements, we choose $\mathrm{n}_{1}(x)$ from it freely.

If $x=y_{i}$ for some $i$, then $\mathfrak{n}_{1}(x)=x$. If $x \neq y_{i}$ for any $i$, then there is $\tilde{x}$ such that

$$
d(\tilde{x}, x)<\frac{\delta_{1} r}{80}+n^{22} \eta^{\frac{1}{3 n}} r \quad \text { and } \quad d(\tilde{x}, p)<\frac{r}{1280}-\frac{\delta_{1} r}{80}-n^{22} \eta^{\frac{1}{3 n}} r .
$$

From (5.27), there exists $y_{i}$ such that $\tilde{x} \in B_{\frac{\delta_{1} r}{80}}\left(y_{i}\right)$, then $d\left(y_{i}, x\right)<\frac{\delta_{1} r}{40}$ and

$$
d\left(y_{i}, p\right) \leq d\left(y_{i}, \tilde{x}\right)+d(\tilde{x}, p)<\frac{r}{1280}-n^{22} \eta^{\frac{1}{3 n}} r,
$$

which implies $y_{i} \in \mathbf{Y}$. From the definition of $\mathfrak{n}_{1}(x)$,

$$
d\left(\mathfrak{n}_{1}(x), x\right) \leq d\left(x, y_{i}\right)<\frac{\delta_{1} r}{40} .
$$

From the definition of $\mathfrak{g}_{1}$ and (5.23), we have

$$
\begin{aligned}
d\left(\mathfrak{g}_{1}(x), \mathfrak{g}_{1}(p)\right) & =d\left(\mathfrak{F}_{\rho_{1}}\left(\mathfrak{n}_{1}(x)\right), \mathfrak{b}_{\rho_{1}}\left(\mathfrak{n}_{1}(p)\right)\right) \leq d\left(\mathfrak{n}_{1}(x), \mathfrak{n}_{1}(p)\right)+\frac{n^{22}}{160} \eta^{\frac{1}{3 n}} r \\
& \leq d\left(\mathfrak{n}_{1}(x), p\right)+d\left(\mathfrak{n}_{1}(p), p\right)+\frac{n^{22}}{160} \eta^{\frac{1}{3 n}} r \\
& \leq \frac{r}{1280}-n^{22} \eta^{\frac{1}{3 n}} r+\frac{\delta_{1} r}{40}+\frac{n^{22}}{160} \eta^{\frac{1}{3 n}} r \\
& <\frac{r}{1280},
\end{aligned}
$$

which implies that $\mathfrak{g}_{1}\left(B \frac{r}{1280}(p)\right) \subset B \frac{r}{1280}(0, \hat{p})$.

Now for any $x_{1}, x_{2} \in B_{\frac{r}{1280}}(p)$, from (5.23) and (5.29), we have

$$
\begin{aligned}
& \left|d\left(\mathfrak{g}_{1}\left(x_{1}\right), \mathfrak{g}_{1}\left(x_{2}\right)\right)-d\left(x_{1}, x_{2}\right)\right| \\
= & \left|d\left(\mathfrak{b}_{\rho_{1}} \circ \mathfrak{n}_{1}\left(x_{1}\right), \mathfrak{5}_{\rho_{1}} \circ \mathfrak{n}_{1}\left(x_{2}\right)\right)-d\left(x_{1}, x_{2}\right)\right| \\
\leq & \mid d\left(\left(\mathfrak{5}_{\rho_{1}} \circ \mathfrak{n}_{1}\left(x_{1}\right), \mathfrak{5}_{\rho_{1}} \circ \mathfrak{n}_{1}\left(x_{2}\right)\right)-d\left(\mathfrak{n}_{1}\left(x_{1}\right), \mathfrak{n}_{1}\left(x_{2}\right)\right) \mid+\frac{\delta_{1} r}{20}\right. \\
\leq & \frac{n^{22}}{160} \eta^{\frac{1}{3 n}} r+\frac{\delta_{1} r}{20} \leq n^{22} \eta^{\frac{1}{3 n}} r .
\end{aligned}
$$

Now to show that $\mathfrak{g}_{1}: B_{\frac{1}{1280} r}(p) \rightarrow B_{\frac{1}{1280} r} r(0, \hat{p})$ is an pointed $\left(\frac{\epsilon r}{3}\right)$-GromovHausdorff approximation, we only need to show that

$$
B_{\frac{r}{1280}}(0, \hat{p}) \subset \mathbf{U}_{\frac{\epsilon r}{3}}\left(\mathfrak{g}_{1}\left(B_{\frac{r}{1280}}(p)\right)\right) .
$$

Step (3). For any $(t, \hat{x}) \in B_{\frac{r}{1280}}(0, \hat{p}) \subset \mathbb{R} \times \mathbf{X}_{1}$, there is $y_{i} \in \mathbf{Y}$ such that $\hat{x}=\mathfrak{P}_{1}\left(y_{i}\right)$. 
If $t+\hat{\rho}_{1}\left(\mathfrak{n}_{1}(p)\right)>\hat{\rho}_{1}\left(y_{i}\right)$, then

$$
t+\hat{\rho}_{1}\left(\mathfrak{n}_{1}(p)\right)-\hat{\rho}_{1}\left(y_{i}\right) \leq \frac{r}{1280}+2 \cdot \frac{r}{320}<\frac{r}{80} .
$$

For $t_{1}=\tilde{\rho}_{1}(p)+\frac{r}{20}$, note

$$
\tilde{\rho}_{1}\left(y_{i}\right) \leq \tilde{\rho}_{1}(p)+d\left(y_{i}, p\right)<\tilde{\rho}_{1}(p)+\frac{r}{1280}<t_{1}-\left(t-\hat{\rho}_{1}\left(\mathfrak{n}_{1}(p)\right)-\hat{\rho}_{1}\left(y_{i}\right)\right) .
$$

There is $\tilde{y} \in \tilde{\rho}_{1}^{-1}\left(t_{1}\right)$ such that $d\left(y_{i}, \tilde{\rho}_{1}^{-1}\left(t_{1}\right)\right)=d\left(y_{i}, \tilde{y}\right)$. Define

$$
z=\gamma_{y_{i}, \tilde{y}}\left(t+\hat{\rho}_{1}\left(\mathfrak{n}_{1}(p)\right)-\hat{\rho}_{1}\left(y_{i}\right)\right),
$$

then

$$
d\left(z, y_{i}\right)=t+\hat{\rho}_{1}\left(n_{1}(p)\right)-\hat{\rho}_{1}\left(y_{i}\right) .
$$

And from (5.18), we have

$$
\tilde{\rho}_{1}(z)=\tilde{\rho}_{1}\left(y_{i}\right)+t+\hat{\rho}_{1}\left(\mathfrak{n}_{1}(p)\right)-\hat{\rho}_{1}\left(y_{i}\right) .
$$

Using (5.34) and (5.21), we get

$$
\begin{aligned}
\left|\hat{\rho}_{1}(z)-\hat{\rho}_{1}\left(\mathfrak{n}_{1}(p)\right)-t\right| & =\left|\rho_{1}(z)-t_{0}-\hat{\rho}_{1}\left(\mathfrak{n}_{1}(p)\right)-t\right| \leq\left|\tilde{\rho}_{1}(z)-t_{0}-\hat{\rho}_{1}\left(\mathfrak{n}_{1}(p)\right)-t\right|+\epsilon_{1} r \\
& =\left|\tilde{\rho}_{1}\left(y_{i}\right)-\hat{\rho}_{1}\left(y_{i}\right)-t_{0}\right|+\epsilon_{1} r \leq\left|\rho_{1}\left(y_{i}\right)-\hat{\rho}_{1}\left(y_{i}\right)-t_{0}\right|+2 \epsilon_{1} r=2 \epsilon_{1} r .
\end{aligned}
$$

Note

$$
\begin{aligned}
d(z, p) & \leq d\left(p, y_{i}\right)+d\left(y_{i}, z\right) \leq \frac{r}{1280}+\left(t+\hat{\rho}_{1}\left(\mathfrak{n}_{1}(p)\right)-\hat{\rho}_{1}\left(y_{i}\right)\right) \\
& <\frac{r}{1280}+\left(\frac{r}{1280}+\frac{r}{1280}\right) \leq \frac{r}{320} .
\end{aligned}
$$

From (5.27), there is $y_{i_{1}}$ such that $d\left(z, y_{i_{1}}\right)<\frac{\delta_{1}}{80} r$, using (5.35) and (5.33),

$$
\begin{aligned}
d\left(\mathfrak{P}_{1}\left(y_{i_{1}}\right), \hat{x}\right) & =\sqrt{d\left(\left(\mathfrak{b}_{\rho_{1}}\left(y_{i_{1}}\right), \mathfrak{5}_{\rho_{1}}\left(y_{i}\right)\right)^{2}-\left|\hat{\rho}_{1}\left(y_{i_{1}}\right)-\hat{\rho}_{1}\left(y_{i}\right)\right|^{2}\right.} \\
& \leq \sqrt{d\left(y_{i_{1}}, y_{i}\right)^{2}-\left|\hat{\rho}_{1}(z)-\hat{\rho}_{1}\left(y_{i}\right)\right|^{2}}+\frac{n^{22}}{160} \eta^{\frac{1}{3 n}} r+\frac{\delta_{1}}{80} r \\
& \leq \sqrt{d\left(z, y_{i}\right)^{2}-\left|t+\hat{\rho}_{1}\left(\mathfrak{n}_{1}(p)\right)-\hat{\rho}_{1}\left(y_{i}\right)\right|^{2}}+n^{22} \eta^{\frac{1}{3 n}} r=n^{22} \eta^{\frac{1}{3 n}} r .
\end{aligned}
$$

Using (5.29), (5.23), (5.35) and (5.36), we have

$$
\begin{aligned}
d(z, p) & \leq d\left(y_{i_{1}}, \mathfrak{n}_{1}(p)\right)+\frac{\delta_{1} r}{20} \leq d\left(\mathfrak{b}_{\rho_{1}}\left(y_{i_{1}}\right), \mathfrak{b}_{\rho_{1}}\left(\mathfrak{n}_{1}(p)\right)\right)+\frac{\delta_{1} r}{20}+\frac{n^{22}}{160} \eta^{\frac{1}{3 n}} r \\
& \leq \sqrt{\left|\hat{\rho}_{1}\left(y_{i_{1}}\right)-\hat{\rho}_{1}\left(\mathfrak{n}_{1}(p)\right)\right|^{2}+d\left(\mathfrak{P}_{1}\left(y_{i_{1}}\right), \hat{p}\right)^{2}}+\frac{\delta_{1} r}{20}+\frac{n^{22}}{160} \eta^{\frac{1}{3 n}} r \\
& \leq \sqrt{\left|\hat{\rho}_{1}(z)-\hat{\rho}_{1}\left(\mathfrak{n}_{1}(p)\right)\right|^{2}+d(\hat{x}, \hat{p})^{2}}+\frac{\delta_{1} r}{10}+2 \cdot n^{22} \eta^{\frac{1}{3 n}} r \\
& \leq \sqrt{t^{2}+d(\hat{x}, \hat{p})^{2}}+n^{23} \eta^{\frac{1}{3 n}} r \\
& <\frac{r}{1280}+n^{23} \eta^{\frac{1}{3 n}} r .
\end{aligned}
$$


Hence we can find $y$ such that

$$
d(z, y) \leq n^{23} \eta^{\frac{1}{3 n}} r+\delta_{1} r \quad \text { and } \quad d(y, p)<\frac{r}{1280}-\delta_{1} r .
$$

From (5.27), there is $y_{i_{2}}$ such that $y \in B_{\frac{\delta_{1} r}{80}}\left(y_{i_{2}}\right)$, then $y_{i_{2}} \in B_{\frac{r}{1280}}(p)$ and

$$
d\left(z, y_{i_{2}}\right)<n^{23} \eta^{\frac{1}{3 n}} r+\delta_{1} r+\frac{\delta_{1} r}{80} \leq n^{24} \eta^{\frac{1}{3 n}} r .
$$

Now using (5.35), similar as (5.36), we get

$$
\begin{aligned}
d\left(\mathfrak{g}_{1}\left(y_{i_{2}}\right),(t, \hat{x})\right) & =d\left(\mathfrak{b}_{\rho_{1}}\left(y_{i_{2}}\right),\left(t+\hat{\rho}_{1}\left(\mathfrak{n}_{1}(p)\right), \hat{x}\right)\right) \\
& \leq\left|\hat{\rho}_{1}\left(y_{i_{2}}\right)-t-\hat{\rho}_{1}\left(\mathfrak{n}_{1}(p)\right)\right|+d\left(\mathfrak{P}_{1}\left(y_{i_{2}}\right), \hat{x}\right) \\
& \leq\left|\hat{\rho}_{1}(z)-t-\hat{\rho}_{1}\left(\mathfrak{n}_{1}(p)\right)\right|+2 \cdot n^{24} \eta^{\frac{1}{3 n}} r \leq n^{25} \eta^{\frac{1}{3 n}} r .
\end{aligned}
$$

If $t+\hat{\rho}_{1}\left(\mathfrak{n}_{1}(p)\right) \leq \hat{\rho}_{1}\left(y_{i}\right)$, we get $\mathfrak{b}_{\rho_{1}}\left(\gamma_{\mathfrak{P}_{1}\left(y_{i}\right), y_{i}}\left(t+\hat{\rho}_{1} \circ \mathfrak{n}_{1}(p)\right)\right)=\left(t+\hat{\rho}_{1}\left(\mathfrak{n}_{1}(p)\right), \hat{x}\right)$, let $z_{0}=\gamma_{\mathfrak{P}_{1}\left(y_{i}\right), y_{i}}\left(t+\hat{\rho}_{1}\left(\mathfrak{r}_{1}(p)\right)\right)$, then

$$
\begin{aligned}
& d\left(z_{0}, y_{i}\right)=\hat{\rho}_{1}\left(z_{0}\right)-\hat{\rho}_{1}\left(y_{i}\right)=t+\hat{\rho}_{1}\left(\mathfrak{n}_{1}(p)\right) \\
& d\left(z_{0}, p\right) \leq d\left(p, y_{i}\right)+d\left(y_{i}, z_{0}\right)<\frac{r}{320} .
\end{aligned}
$$

Now using (5.29) and (5.39), we have

$$
\begin{aligned}
d\left(\mathfrak{P}_{1} \circ \mathfrak{n}_{1}\left(z_{0}\right), \hat{x}\right) & =\sqrt{d\left(\mathfrak{b}_{\rho_{1}} \circ \mathfrak{n}_{1}\left(z_{0}\right), \mathfrak{b}_{\rho_{1}}\left(y_{i}\right)\right)^{2}-\left|\hat{\rho}_{1} \circ \mathfrak{n}_{1}\left(z_{0}\right)-\hat{\rho}_{1}\left(y_{i}\right)\right|^{2}} \\
& \leq \sqrt{d\left(\mathfrak{n}_{1}\left(z_{0}\right), y_{i}\right)^{2}-\left|\hat{\rho}_{1}\left(z_{0}\right)-\hat{\rho}_{1}\left(y_{i}\right)\right|^{2}}+\frac{n^{22}}{160} \eta^{\frac{1}{3 n}} r+\frac{\delta_{1} r}{80} \\
& \leq \sqrt{d\left(z_{0}, y_{i}\right)^{2}-\left|t+\hat{\rho}_{1}\left(\mathfrak{n}_{1}(p)\right)\right|^{2}}+n^{22} \eta^{\frac{1}{3 n}} r=n^{22} \eta^{\frac{1}{3 n}} r .
\end{aligned}
$$

Then using (5.40), similar as (5.37) we get $d\left(z_{0}, p\right)<\frac{r}{1280}+n^{23} \eta^{\frac{1}{3 n}} r$. The rest argument is similar to get (5.38), we can find $y_{j_{2}} \in B_{\frac{r}{1280}}(p)$ such that

$$
d\left(\mathfrak{g}_{1}\left(y_{j_{2}}\right),(t, \hat{x})\right) \leq n^{25} \eta^{\frac{1}{3 n}} r .
$$

From above, choose $\eta=n^{-100 n} \epsilon^{3 n}, \epsilon_{1}=n^{-301 n^{2}} \epsilon^{9 n^{2}}<2^{-n} \eta^{3 n}$, and finally $\delta=n^{-700 n^{4}} \epsilon^{18 n^{4}}<2^{-100 n^{2}} \epsilon_{1}^{2 n^{2}}$, we get the pointed ( $\left.\frac{\epsilon r}{3}\right)$-Gromov-Hausdorff approximation $\mathfrak{g}_{1}: B_{\frac{r}{1280}}(p) \rightarrow B_{\frac{r}{1280}}(0, \hat{p})$.

Step (4). Note $\mathbf{b}_{1}(p)=0$, from (5.20), we have $\left|\rho_{1}(p)\right|<\epsilon_{1} r$, then

$$
\begin{aligned}
\left|\hat{\rho}_{1} \circ \mathfrak{n}_{1}(p)+t_{0}\right| & \leq\left|\hat{\rho}_{1}(p)+t_{0}\right|+\left|d\left(p, \mathfrak{n}_{1}(p)\right)\right| \leq\left|\rho_{1}(p)\right|+\frac{\delta_{1} r}{40} \leq \epsilon_{1} r+\frac{\delta_{1} r}{40} \\
& <\frac{1}{2} n^{-29} \epsilon r .
\end{aligned}
$$


Define $\mathcal{P}_{1}=\mathfrak{P}_{1} \circ \mathrm{n}_{1}$, for $f_{1}=\left(\mathbf{b}_{1}, \mathcal{P}_{1}\right)$, then from (5.42) and (5.20),

$$
\begin{aligned}
d\left(f_{1}(x), \mathfrak{g}_{1}(x)\right) & =\left|\mathbf{b}_{1}(x)-\hat{\rho}_{1} \circ \mathfrak{n}_{1}(x)+\hat{\rho}_{1} \circ \mathfrak{n}_{1}(p)\right| \\
& \leq\left|\mathbf{b}_{1}(x)-\rho_{1}(x)\right|+\left|\rho_{1}(x)-\hat{\rho}_{1} \circ \mathfrak{n}_{1}(x)+\hat{\rho}_{1} \circ \mathfrak{n}_{1}(p)\right| \\
& \leq \epsilon_{1} r+\left|\hat{\rho}_{1}(x)+t_{0}-\hat{\rho}_{1} \circ \mathfrak{n}_{1}(x)+\hat{\rho}_{1} \circ \mathfrak{n}_{1}(p)\right| \\
& \leq \epsilon_{1} r+\left|\hat{\rho}_{1}(x)-\hat{\rho}_{1} \circ \mathfrak{n}_{1}(x)\right|+\left|t_{0}+\hat{\rho}_{1} \circ \mathfrak{n}_{1}(p)\right| \\
& \leq \epsilon_{1} r+d\left(x, \mathfrak{n}_{1}(x)\right)+\frac{1}{2} n^{-29} \epsilon r \\
& <n^{-29} \epsilon r .
\end{aligned}
$$

Now from (5.30) and (5.43),

$$
\begin{aligned}
\left|d\left(f_{1}\left(x_{1}\right), f_{1}\left(x_{2}\right)\right)-d\left(x_{1}, x_{2}\right)\right| & \leq\left|d\left(\mathfrak{g}_{1}\left(x_{1}\right), \mathfrak{g}_{1}\left(x_{2}\right)\right)-d\left(x_{1}, x_{2}\right)\right|+2 \cdot n^{-29} \epsilon r \\
& \leq n^{22} \eta^{\frac{1}{3 n}} r+n^{-29} \epsilon r \leq \frac{1}{3} \epsilon r .
\end{aligned}
$$

From (5.38), (5.41) and (5.43), we have

$$
d\left(f_{1}\left(y_{i_{2}}\right),(t, \hat{x})\right) \leq n^{-29} \epsilon r+n^{25} \eta^{\frac{1}{3 n}} r \leq \frac{\epsilon}{3} r .
$$

From (5.44) and (5.45), we obtain that $f_{1}$ is an $\left(\frac{\epsilon}{3} r\right)$-Gromov-Hausdorff approximation.

Step (5). We will only prove the case $k=2$, the rest cases have similar argument. From the case $k=1$, for $\mathbf{b}_{i}, i=1,2$, there is corresponding pointed $\left(\frac{\epsilon r}{3}\right)$-GromovHausdorff approximation $f_{\mathbf{b}_{i}}: B_{\frac{r}{1280}}(p) \rightarrow B_{\frac{r}{1280}}\left(0, \hat{p}_{i}\right) \subset \mathbb{R} \times \mathbf{X}_{\mathbf{b}_{i}}$. We assume $f_{\mathbf{b}_{i}}=$ $\left(\mathbf{b}_{i}, \mathcal{P}_{\mathbf{b}_{i}}\right)$. We will prove the map $f_{2}(x)=\left(\mathbf{b}_{1}(x), \mathbf{b}_{2}(x), \mathcal{P}_{\mathbf{b}_{2}} \circ \mathcal{P}_{\mathbf{b}_{1}}(x)\right): B_{\frac{r}{1280}}(p) \rightarrow$ $B \frac{r}{1280}\left(0, \hat{p}_{i}\right) \subset \mathbb{R}^{2} \times \mathbf{X}_{2}$ is an pointed $\left(\frac{2 \epsilon r}{3}\right)$-Gromov-Hausdorff approximation.

From the first variation formula of arc length, for $x \in P_{\eta, \mathbf{b}_{1}, \mathbf{b}_{2}}^{r, \rho_{1}} \cap Q_{\eta, \mathbf{b}_{1}}^{r, \rho_{1}} \cap Q_{\eta, \mathbf{b}_{2}}^{r, \rho_{2}, \rho_{1}}$, let $\breve{x}=n_{1}(x)$, then we have

$$
\begin{aligned}
& \left|\mathbf{b}_{2}(x)-\mathbf{b}_{2} \circ \mathcal{P}_{\mathbf{b}_{1}}(x)\right| \leq\left|\rho_{2}(x)-\rho_{2} \circ \mathfrak{P}_{1}(\check{x})\right|+2 \epsilon_{1} r \\
\leq & \left|\rho_{2}(\check{x})-\rho_{2} \circ \mathfrak{P}_{1}(\check{x})\right|+2 \epsilon_{1} r+\frac{\delta_{1} r}{40} \\
= & \left|\hat{\rho}_{2}(\check{x})-\hat{\rho}_{2}\left(\sigma_{\check{x}, 1}(0)\right)\right|+2 \epsilon_{1} r+\frac{\delta_{1} r}{40} \\
= & \left|\int_{0}^{\hat{\rho}_{1}(\check{x})-\eta r}\left\langle\nabla \hat{\rho}_{1}, \nabla \hat{\rho}_{2}\right\rangle\left(\sigma_{\check{x}, 1}(s)\right) d s\right|+2 \epsilon_{1} r+\frac{\delta_{1} r}{40} \\
\leq & \int_{0}^{\hat{\rho}_{1}(\breve{x})-\eta r}\left(\left|\left\langle\nabla \mathbf{b}_{1}, \nabla \mathbf{b}_{2}\right\rangle\right|+\left|\nabla\left(\hat{\rho}_{1}-\mathbf{b}_{1}\right)\right|+\left|\nabla\left(\hat{\rho}_{2}-\mathbf{b}_{2}\right)\right|\right)\left(\sigma_{\check{x}, 1}(s)\right) d s \\
& +2 \epsilon_{1} r+\frac{\delta_{1} r}{40} \\
\leq & 3 \eta r+2 \epsilon_{1} r+\frac{\delta_{1} r}{40} \leq \frac{1}{2 n} \epsilon r .
\end{aligned}
$$


Let $\mathfrak{w}(x)=\left(\mathbf{b}_{1}(x), \mathbf{b}_{2}\left(\mathcal{P}_{\mathbf{b}_{1}}(x)\right), \mathcal{P}_{\mathbf{b}_{2}}\left(\mathcal{P}_{\mathbf{b}_{1}}(x)\right)\right)$ for any $x, y \in B_{\frac{r}{1280}}(p)$,

$$
\begin{aligned}
|d(\mathfrak{w}(x), \mathfrak{w}(y))-d(x, y)| & \leq\left|d(\mathfrak{w}(x), \mathfrak{w}(y))-d\left(f_{\mathbf{b}_{1}}(x), f_{\mathbf{b}_{1}}(y)\right)\right|+\frac{\epsilon r}{3} \\
& \leq \frac{\left|d\left(f_{\mathbf{b}_{2}} \circ \mathcal{P}_{\mathbf{b}_{1}}(x), f_{\mathbf{b}_{2}} \circ \mathcal{P}_{\mathbf{b}_{1}}(y)\right)^{2}-d\left(\mathcal{P}_{\mathbf{b}_{1}}(x), \mathcal{P}_{\mathbf{b}_{1}}(y)\right)^{2}\right|}{d(\mathfrak{w}(x), \mathfrak{w}(y))+d\left(f_{\mathbf{b}_{1}}(x), f_{\mathbf{b}_{1}}(y)\right)}+\frac{\epsilon r}{3} \\
& \leq\left|d\left(f_{\mathbf{b}_{2}} \circ \mathcal{P}_{\mathbf{b}_{1}}(x), f_{\mathbf{b}_{2}} \circ \mathcal{P}_{\mathbf{b}_{1}}(y)\right)-d\left(\mathcal{P}_{\mathbf{b}_{1}}(x), \mathcal{P}_{\mathbf{b}_{1}}(y)\right)\right|+\frac{\epsilon r}{3} \\
& \leq \frac{2 \epsilon r}{3} .
\end{aligned}
$$

Now we have

$$
\begin{aligned}
& \left|d\left(f_{2}(x), f_{2}(y)\right)-d(x, y)\right| \\
\leq & \left|d\left(f_{2}(x), f_{2}(y)\right)-d(\mathfrak{w}(x), \mathfrak{w}(y))\right|+|d(\mathfrak{w}(x), \mathfrak{w}(y))-d(x, y)| \\
\leq & d\left(f_{2}(x), \mathfrak{w}(x)\right)+d\left(f_{2}(y), \mathfrak{w}(y)\right)+\frac{2 \epsilon r}{3} \\
\leq & \left|\mathbf{b}_{2}(x)-\mathbf{b}_{2} \circ \mathcal{P}_{\mathbf{b}_{1}}(x)\right|+\left|\mathbf{b}_{2}(y)-\mathbf{b}_{2} \circ \mathcal{P}_{\mathbf{b}_{1}}(y)\right|+\frac{2 \epsilon r}{3} \\
\leq & \frac{\epsilon r}{n}+\frac{2 \epsilon r}{3} \leq \epsilon r .
\end{aligned}
$$

The rest argument is similar as in the case $k=1$.

PaRt III. Covering Groups of RiEMANNIAN MANIFolds with $R c \geq 0$

\section{SQueEze Lemma and Dimension induction on harmonic Functions}

In this section, unless otherwise mentioned, we assume $\varphi: \tilde{M}^{n} \rightarrow M^{n}$ is the covering map with covering group $\Gamma$ such that $M^{n}=\tilde{M}^{n} / \Gamma$, where $\left(\tilde{M}^{n}, \tilde{g}\right)$ and $\left(M^{n}, g\right)$ are two complete Riemannian manifolds and the metric $g$ is the quotient metric of $\tilde{g}$ with respect to group action of $\Gamma$.

We include the definition of quotient metric here for convenience.

Definition 6.1. Consider a subgroup $G \subset \operatorname{Isom}(\mathbf{X})$, where $\mathbf{X}$ is a metric space, for every $\bar{x}, \bar{y} \in \mathbf{X} / G$, set the quotient metric $\bar{d}$ on $\mathbf{X} / G$ by:

$$
\bar{d}(\bar{x}, \bar{y})=\inf \{d(x, y): x \in \bar{x}, y \in \bar{y}\}=\inf \{d(x, g y): g \in G\} .
$$

For any function $f$ defined on a domain $\mathbf{D} \subset M^{n}$, we define

$$
\mathcal{L}(f)(\tilde{x}):=f(\varphi(\tilde{x})), \quad \forall \tilde{x} \in \varphi^{-1}(\mathbf{D}) .
$$

For $\tilde{p}, \tilde{q} \in \tilde{M}^{n}$ and any $s>0$, we define

$$
\Gamma_{\tilde{p}}(s)=\{\gamma \in \Gamma \mid d(\tilde{p}, \gamma \tilde{p}) \leq s\} .
$$

Similarly we can define $\Gamma_{\tilde{q}}(s)$. When the point is fixed and clear in the context, we use $\Gamma(s)$ instead of $\Gamma_{\tilde{p}}(s)$ for simplicity.

The following Lemma is motivated by the use of the canonical fundamental domain $\mathcal{F}$ in [And90], and is needed for the proof of the squeeze lemma. 
Lemma 6.2. For any function $f \geq 0$ defined on $B_{r}(p) \subset M^{n}, \tilde{p}$ is one lift of $p$ and $B_{r}(\tilde{p})$ is the geodesic ball centered at $\tilde{p}$ with radius $r$ in $\tilde{M}^{n}$. Then

$$
B_{r}(\tilde{p}) \subset \varphi^{-1}\left(B_{r}(p)\right) \quad \text { and } \quad f_{B_{r}(\tilde{p})} \mathcal{L}(f) \leq 4^{n} f_{B_{r}(p)} f .
$$

Proof: Let $\tilde{\Omega}=\varphi^{-1}\left(B_{r}(p)\right)$. For any $\tilde{y} \in B_{r}(\tilde{p})$,

$$
d(p, \varphi(\tilde{y}))=\inf _{h \in \Gamma} d(\tilde{p}, h \tilde{y}) \leq d(\tilde{p}, \tilde{y})<r,
$$

which implies $\varphi\left(B_{r}(\tilde{p})\right) \subset B_{r}(p)$, then

$$
B_{r}(\tilde{p}) \subset \varphi^{-1}\left(B_{r}(p)\right)=\tilde{\Omega} .
$$

We choose a measurable section $s: B_{r}(p) \rightarrow B_{r}(\tilde{p})$, such that $\varphi(s(x))=x$ for any $x \in B_{r}(p)$. Let $T=s\left(B_{r}(p)\right)$, then we have $f_{B_{r}(p)} f=f_{T} \mathcal{L}(f)$. Let $S$ be the union of $g(T)$ over all $g \in \Gamma$ such that $g(T) \cap B_{r}(\tilde{p}) \neq \emptyset$. Then from $\operatorname{diam}(T) \leq 2 r$, we obtain $S \subseteq B_{3 r}(\tilde{p})$. And we also have $f_{B_{r}(p)} f=f_{S} \mathcal{L}(f)$ and $B_{r}(\tilde{p}) \subseteq S$.

From the Bishop-Gromov volume comparison theorem, we know $V\left(B_{3 r}(\tilde{p})\right) \leq$ $3^{n} V\left(B_{r}(\tilde{p})\right)$. Note $B_{r}(\tilde{p}) \subseteq S \subseteq B_{3 r}(\tilde{p})$, we have $V(S) \leq 3{ }^{n} V\left(B_{r}(\tilde{p})\right)$, hence

$$
f_{B_{r}(\tilde{p})} \mathcal{L}(f) \leq \frac{1}{V\left(B_{r}(\tilde{p})\right)} \int_{S} \mathcal{L}(f)=\frac{V(S)}{V\left(B_{r}(\tilde{p})\right)} f_{S} \mathcal{L}(f) \leq 4^{n} f_{B_{r}(p)} f .
$$

Before we state and prove our squeeze lemma, we would like to include the following well-known result and its proof here, because the squeeze lemma can be looked at the Gromov-Hausdorff perturbation version of the following Lemma.

Lemma 6.3. For a locally compact, pointed length space $(\mathcal{Z}, q)$, assume $G$ is a closed subgroup of the isometry group $\operatorname{Isom}(\mathcal{Z})$ and $\mathcal{Z} / G=\mathbf{K}$, where $\mathbf{K}$ is a compact metric space with $\operatorname{diam}(\mathbf{K})=r_{0}$. Then for any $\epsilon>0$, we have

$$
G=\left\langle G\left(2 r_{0}+\epsilon\right)\right\rangle .
$$

Proof: For any $g_{1} \in G$, because $\mathcal{Z}$ is a length space, there is a segment $\gamma_{q, g_{1} q}$ from $q$ to $g_{1} q$. Then one can find a middle point $x \in \mathcal{Z}$ of the segment $\gamma_{q, g_{1} q}$ such that $d(x, q)=d\left(x, g_{1} q\right)=\frac{1}{2} d\left(q, g_{1} q\right)$. Since $\mathcal{Z} / G=\mathbf{K}$ and $G$ is a closed subgroup, one can find $g_{2} \in G$ such that $d\left(g_{2} q, x\right) \leq r_{0}$. Then we have

$$
\begin{aligned}
& d\left(q, g_{2} q\right) \leq d(q, x)+d\left(x, g_{2} q\right) \leq \frac{1}{2} d\left(q, g_{1} q\right)+r_{0} \\
& d\left(q, g_{2}^{-1} g_{1} q\right)=d\left(g_{2} q, g_{1} q\right) \leq d\left(g_{2} q, x\right)+d\left(x, g_{1} q\right) \leq r_{0}+\frac{1}{2} d\left(q, g_{1} q\right) .
\end{aligned}
$$

Assume $r=d\left(q, g_{1} q\right)$, then we have

$$
g_{2} \in G\left(\frac{r}{2}+r_{0}\right), \quad g_{2}^{-1} g_{1} \in G\left(\frac{r}{2}+r_{0}\right),
$$

which implies $g_{1} \in\left\langle G\left(\frac{r}{2}+r_{0}\right)\right\rangle$. Using that $G$ is a closed subgroup, by induction on $r$ we get $g_{1} \in\left\langle G\left(2 r_{0}\right)\right\rangle$, and the conclusion follows. 
Lemma 6.4 (Squeeze Lemma). For any $\epsilon>0, \delta=n^{-900 n^{4}} \epsilon^{40 n^{4}}$, integer $0 \leq k \leq n$, if there exist harmonic functions $\left\{\mathbf{b}_{i}\right\}_{i=1}^{k}$ defined on $B_{r}(p)$, satisfying $\mathbf{b}_{i}(p)=0$ and

$$
\sup _{\substack{B r(p) \\ i=1, \cdots, k}}\left|\nabla \mathbf{b}_{i}\right| \leq 2, \quad \quad \sup _{t \leq r} f_{B_{t}(p)} \sum_{i, j=1}^{k}\left|\left\langle\nabla \mathbf{b}_{i}, \nabla \mathbf{b}_{j}\right\rangle-\delta_{i j}\right| \leq \delta,
$$

then there is a family of $(\epsilon s)$-Gromov-Hausdorff approximation for $s \in\left(0,10 r_{c}\right]$,

$$
f_{s}=\left(\mathbf{b}_{1}, \cdots, \mathbf{b}_{k}, \mathcal{P}_{s}\right): B_{s}(p) \rightarrow B_{s}\left(0, \hat{p}_{s}\right) \subset \mathbb{R}^{k} \times \mathbf{X}_{k, s},
$$

where $r_{c}=\frac{r}{12800}$. And let $\operatorname{diam}\left(B_{r_{c}}\left(\hat{p}_{10 r_{c}}\right)\right)=r_{0}$, we have

$$
\Gamma\left(r_{c}\right) \subset\left\langle\Gamma\left(\epsilon r_{c}+2 r_{0}\right)\right\rangle .
$$

Proof: In the proof, we can assume that $r_{0} \leq \frac{1}{2} r_{c}$, otherwise the conclusion follows directly. Let $\tilde{\mathbf{b}}_{i}=\mathcal{L}\left(\mathbf{b}_{i}\right), i=1, \cdots, k$, from (6.2) and Lemma 6.2, we have $\mathbf{b}_{i}(p)=\tilde{\mathbf{b}}_{i}(\tilde{p})=0$ and

$$
\sup _{\substack{B_{r}(\tilde{p}) \\ i=1, \cdots, k}}\left|\nabla \tilde{\mathbf{b}}_{i}\right| \leq 2, \quad \quad \sup _{t \leq r} f_{B_{t}(\tilde{p})} \sum_{i, j=1}^{k}\left|\left\langle\nabla \tilde{\mathbf{b}}_{i}, \nabla \tilde{\mathbf{b}}_{j}\right\rangle-\delta_{i j}\right| \leq 4^{n} \delta .
$$

For $0<\epsilon_{1}<1$ to be determined later, from Theorem 2.13, if we assume

$$
\delta \leq 4^{-n} n^{-700 n^{4}} \epsilon_{1}^{18 n^{4}},
$$

there are two family of $\left(\epsilon_{1} s\right)$-Gromov-Hausdorff approximation for $s \in\left(0,10 r_{c}\right]$,

$$
\begin{aligned}
& f_{s}=\left(\mathbf{b}_{1}, \cdots, \mathbf{b}_{k}, \mathcal{P}_{s}\right): B_{s}(p) \rightarrow B_{s}\left(0, \hat{p}_{s}\right) \subset \mathbb{R}^{k} \times \mathbf{X}_{k, s} \\
& \tilde{f}_{s}=\left(\tilde{\mathbf{b}}_{1}, \cdots, \tilde{\mathbf{b}}_{k}, \tilde{\mathcal{P}}_{s}\right): B_{s}(\tilde{p}) \rightarrow B_{s}\left(0, \check{p}_{s}\right) \subset \mathbb{R}^{k} \times \tilde{\mathbf{X}}_{k, s} .
\end{aligned}
$$

From Lemma 2.11, there is an $\left(30 \epsilon_{1} r_{c}\right)$-Gromov-Hausdorff approximation

$$
\Phi: B_{10 r_{c}}\left(0, \check{p}_{10 r_{c}}\right) \rightarrow B_{10 r_{c}}(\tilde{p}),
$$

where $B_{10 r_{c}}\left(0, \check{p}_{10 r_{c}}\right) \subset \mathbb{R}^{k} \times \tilde{\mathbf{X}}_{k, 10 r_{c}}$.

For any $\gamma \in \Gamma\left(r_{c}\right)$, we have

$$
\begin{aligned}
d\left(\tilde{\mathcal{P}}_{10 r_{c}}(\tilde{p}), \tilde{\mathcal{P}}_{10 r_{c}}(\gamma \tilde{p})\right) & \leq d\left(\tilde{f}_{10 r_{c}}(\tilde{p}), \tilde{f}_{10 r_{c}}(\gamma \tilde{p})\right)<d(\tilde{p}, \gamma \tilde{p})+10 \epsilon_{1} r_{c} \\
& \leq r_{c}+10 \epsilon_{1} r_{c}<2 r_{c} .
\end{aligned}
$$

Also note $\check{p}_{10 r_{c}}=\tilde{\mathcal{P}}_{10 r_{c}}(\tilde{p})$, then we have $\tilde{\mathcal{P}}_{10 r_{c}}(\tilde{p}), \tilde{\mathcal{P}}_{10 r_{c}}(\gamma \tilde{p}) \in B_{2 r_{c}}\left(\check{p}_{10 r_{c}}\right)$.

Apply the argument of Lemma 2.8 to $\tilde{\mathcal{P}}_{10 r_{c}}(\tilde{p})$ and $\tilde{\mathcal{P}}_{10 r_{c}}(\gamma \tilde{p})$, we can obtain $\tilde{z} \in B_{4 r_{c}}\left(\check{p}_{10 r_{c}}\right)$ such that

$$
\begin{aligned}
\left|d\left(\tilde{z}, \tilde{\mathcal{P}}_{10 r_{c}}(\tilde{p})\right)-\frac{1}{2} d\left(\tilde{\mathcal{P}}_{10 r_{c}}(\tilde{p}), \tilde{\mathcal{P}}_{10 r_{c}}(\gamma \tilde{p})\right)\right| & \leq 10 \sqrt{30 \epsilon_{1}} r_{c} \\
\left|d\left(\tilde{z}, \tilde{\mathcal{P}}_{10 r_{c}}(\gamma \tilde{p})\right)-\frac{1}{2} d\left(\tilde{\mathcal{P}}_{10 r_{c}}(\tilde{p}), \tilde{\mathcal{P}}_{10 r_{c}}(\gamma \tilde{p})\right)\right| & \leq 10 \sqrt{30 \epsilon_{1}} r_{c} .
\end{aligned}
$$


Then note $\tilde{\mathbf{b}}_{i}(\tilde{p})=\tilde{\mathbf{b}}_{i}(\gamma \tilde{p})=0$, we have

$$
\begin{aligned}
d\left(\tilde{z}, \check{p}_{10 r_{c}}\right) & =d\left(\tilde{z}, \tilde{\mathcal{P}}_{10 r_{c}}(\tilde{p})\right) \leq \frac{1}{2} d\left(\tilde{\mathcal{P}}_{10 r_{c}}(\tilde{p}), \tilde{\mathcal{P}}_{10 r_{c}}(\gamma \tilde{p})\right)+10 \sqrt{30 \epsilon_{1}} r_{c} \\
& =\frac{1}{2} d\left(\tilde{f}_{10 r_{c}}(\tilde{p}), \tilde{f}_{10 r_{c}}(\gamma \tilde{p})\right)+10 \sqrt{30 \epsilon_{1}} r_{c} \\
& <\frac{1}{2} d(\tilde{p}, \gamma \tilde{p})+70 \sqrt{\epsilon_{1}} r_{c} \\
& \leq \frac{1}{2} r_{c}+70 \sqrt{\epsilon_{1}} r_{c} .
\end{aligned}
$$

Similarly, we have

$$
d\left(\tilde{z}, \tilde{\mathcal{P}}_{10 r_{c}}(\gamma \tilde{p})\right)<\frac{1}{2} d(\tilde{p}, \gamma \tilde{p})+70 \sqrt{\epsilon_{1}} r_{c} .
$$

From 6.7), we get that $(0, \tilde{z}) \in B_{\frac{1}{2} r_{c}+70 \sqrt{\epsilon_{1}} r_{c}}\left(0, \check{p}_{10 r_{c}}\right)$. Because $\tilde{f}_{10 r_{c}}$ is an $\left(10 \epsilon_{1} r_{c}\right)$ Gromov-Hausdorff approximation, there is $z_{1} \in B_{10 r_{c}}(\tilde{p})$ such that

$$
d\left(\tilde{f}_{10 r_{c}}\left(z_{1}\right),(0, \tilde{z})\right)<10 \epsilon_{1} r_{c} .
$$

Now we have

$$
\begin{aligned}
d\left(z_{1}, \tilde{p}\right) & \leq d\left(\tilde{f}_{10 r_{c}}\left(z_{1}\right), \tilde{f}_{10 r_{c}}(\tilde{p})\right)+10 \epsilon_{1} r_{c} \\
& \leq d\left(\tilde{f}_{10 r_{c}}\left(z_{1}\right),(0, \tilde{z})\right)+d\left((0, \tilde{z}), \tilde{f}_{10 r_{c}}(\tilde{p})\right)+10 \epsilon_{1} r_{c} \\
& <\frac{1}{2} r_{c}+70 \sqrt{\epsilon_{1}} r_{c}+20 \epsilon_{1} r_{c} \\
& \leq \frac{1}{2} r_{c}+90 \sqrt{\epsilon_{1}} r_{c} .
\end{aligned}
$$

Assume $z_{0}=\varphi\left(z_{1}\right)$, we have $d\left(z_{0}, p\right) \leq d\left(z_{1}, \tilde{p}\right)<\frac{1}{2} r_{c}+90 \sqrt{\epsilon_{1}} r_{c}$. Then

$$
\begin{aligned}
d\left(\mathcal{P}_{10 r_{c}}\left(z_{0}\right), \hat{p}_{10 r_{c}}\right) & =d\left(\mathcal{P}_{10 r_{c}}\left(z_{0}\right), \mathcal{P}_{10 r_{c}}(p)\right) \leq d\left(f_{10 r_{c}}\left(z_{0}\right), f_{10 r_{c}}(p)\right) \\
& \leq d\left(z_{0}, p\right)+\epsilon_{1} \cdot\left(10 r_{c}\right)<\frac{1}{2} r_{c}+100 \sqrt{\epsilon_{1}} r_{c}<r_{c},
\end{aligned}
$$

which implies $\mathcal{P}_{10 r_{c}}\left(z_{0}\right) \in B_{r_{c}}\left(\hat{p}_{10 r_{c}}\right)$. From the assumption $\operatorname{diam}\left(B_{r_{c}}\left(\hat{p}_{10 r_{c}}\right)\right)=r_{0}$,

$$
d\left(\mathcal{P}_{10 r_{c}}\left(z_{0}\right), \hat{p}_{10 r_{c}}\right) \leq r_{0} .
$$

Let $\tilde{\mathbf{b}}=\left(\tilde{\mathbf{b}}_{1}, \cdots, \tilde{\mathbf{b}}_{k}\right)$ and $\mathbf{b}=\left(\mathbf{b}_{1}, \cdots, \mathbf{b}_{k}\right)$, from 6.11) and 6.9), we obtain

$$
\begin{aligned}
d\left(z_{0}, p\right) & \leq 10 \epsilon_{1} r_{c}+d\left(f_{10 r_{c}}\left(z_{0}\right), f_{10 r_{c}}(p)\right) \\
& \leq 10 \epsilon_{1} r_{c}+d\left(\mathbf{b}\left(z_{0}\right), 0\right)+d\left(\mathcal{P}_{10 r_{c}}\left(z_{0}\right), \mathcal{P}_{10 r_{c}}(p)\right) \\
& \leq 10 \epsilon_{1} r_{c}+d\left(\tilde{\mathbf{b}}\left(z_{1}\right), 0\right)+d\left(\mathcal{P}_{10 r_{c}}\left(z_{0}\right), \hat{p}_{10 r_{c}}\right) \\
& \leq 20 \epsilon_{1} r_{c}+r_{0} .
\end{aligned}
$$

There exists $\gamma_{2} \in \Gamma$ such that $d\left(z_{1}, \gamma_{2} \tilde{p}\right)=d\left(z_{0}, p\right)<r_{c}$, then from 6.10),

$$
d\left(\tilde{p}, \gamma_{2} \tilde{p}\right) \leq d\left(\tilde{p}, z_{1}\right)+d\left(z_{1}, \gamma_{2} \tilde{p}\right)<2 r_{c} .
$$


We have $\gamma_{2} \tilde{p} \in B_{2 r_{c}}(\tilde{p})$, from (6.9) and (6.12), we obtain

$$
\begin{aligned}
d\left((0, \tilde{z}), \tilde{f}_{10 r_{c}}\left(\gamma_{2} \tilde{p}\right)\right) & <10 \epsilon_{1} r_{c}+d\left(\tilde{f}_{10 r_{c}}\left(z_{1}\right), \tilde{f}_{10 r_{c}}\left(\gamma_{2} \tilde{p}\right)\right) \leq 20 \epsilon_{1} r_{c}+d\left(z_{1}, \gamma_{2} \tilde{p}\right) \\
& =20 \epsilon_{1} r_{c}+d\left(z_{0}, p\right) \leq 40 \epsilon_{1} r_{c}+r_{0} .
\end{aligned}
$$

Now from (6.6) and (6.13), we obtain

$$
\begin{aligned}
d\left(\tilde{p}, \gamma_{2} \tilde{p}\right) & \leq d\left(\tilde{f}_{10 r_{c}}(\tilde{p}), \tilde{f}_{10 r_{c}}\left(\gamma_{2} \tilde{p}\right)\right)+10 \epsilon_{1} r_{c} \\
& \leq d\left(\tilde{f}_{10 r_{c}}(\tilde{p}),(0, \tilde{z})\right)+d\left((0, \tilde{z}), \tilde{f}_{10 r_{c}}\left(\gamma_{2} \tilde{p}\right)\right)+10 \epsilon_{1} r_{c} \\
& <d\left(\tilde{z}, \check{p}_{10 r_{c}}\right)+\left(40 \epsilon_{1} r_{c}+r_{0}\right)+10 \epsilon_{1} r_{c} \\
& \leq \frac{1}{2} d(\tilde{p}, \gamma \tilde{p})+\left(120 \sqrt{\epsilon_{1}} r_{c}+r_{0}\right) .
\end{aligned}
$$

Similarly from (6.8) and (6.13), we can get

$$
\begin{aligned}
d\left(\gamma_{2} \tilde{p}, \gamma \tilde{p}\right) & =d\left(\tilde{f}_{10 r_{c}}\left(\gamma_{2} \tilde{p}\right), \tilde{f}_{10 r_{c}}(\gamma \tilde{p})\right)+10 \epsilon_{1} r_{c} \\
& \leq d\left(\tilde{f}_{10 r_{c}}(\gamma \tilde{p}),(0, \tilde{z})\right)+d\left((0, \tilde{z}), \tilde{f}_{10 r_{c}}\left(\gamma_{2} \tilde{p}\right)\right)+10 \epsilon_{1} r_{c} \\
& <d\left(\tilde{z}, \tilde{\mathcal{P}}_{10 r_{c}}(\gamma \tilde{p})\right)+\left(40 \epsilon_{1} r_{c}+r_{0}\right)+10 \epsilon_{1} r_{c} \\
& \leq \frac{1}{2} d(\tilde{p}, \gamma \tilde{p})+\left(120 \sqrt{\epsilon_{1}} r_{c}+r_{0}\right) .
\end{aligned}
$$

Let $\epsilon_{1}=\frac{\epsilon^{2}}{90000}$, from (6.14) and (6.15), we get $\gamma \in\left\langle\Gamma\left(\frac{1}{2} d(\tilde{p}, \gamma \tilde{p})+r_{0}+\frac{120}{300} \epsilon r_{c}\right)\right\rangle$.

From (6.3), we can choose $\delta=n^{-900 n^{4}} \epsilon^{40 n^{4}}$. Now by induction, we have $\gamma \in$ $\left\langle\Gamma\left(\epsilon r_{c}+2 r_{0}\right)\right\rangle$, which implies the conclusion.

Lemma 6.5. If $\epsilon<\frac{1}{10 n^{2}}$, there do not exist $\left\{v_{i}\right\}_{i=1}^{n+1} \subset \mathbb{R}^{n}$ such that

$$
\sup _{1 \leq i<j \leq n+1}\left|\left\langle v_{i}, v_{j}\right\rangle\right| \leq \epsilon \quad \text { and } \quad \sup _{1 \leq i \leq n+1}|| v_{i}|-1| \leq \epsilon .
$$

Proof: To prove the conclusion, we only need to show that $v_{1}, \cdots, v_{n+1}$ are linearly independent. By contradiction, otherwise, without loss of generality, one can assume $v_{n+1}=\sum_{i=1}^{n} a_{i} v_{i}$ and $\left|a_{1}\right|=\max _{i=1, \cdots, n}\left|a_{i}\right|$, where $a_{i} \in \mathbb{R}$. Then from the assumption,

$$
\epsilon \geq\left|\left\langle v_{n+1}, v_{1}\right\rangle\right| \geq\left|a_{1}\right| \cdot\left|v_{1}\right|^{2}-\sum_{i=2}^{n}\left|a_{i}\right| \cdot\left|\left\langle v_{i}, v_{1}\right\rangle\right| \geq\left|a_{1}\right| \cdot[(1-\epsilon)-(n-1) \epsilon],
$$

which implies

$$
\left|a_{1}\right| \leq \frac{\epsilon}{1-n \epsilon} .
$$

On the other hand, from (6.16) and $\epsilon<\frac{1}{10 n^{2}}$, we can get

$$
\left|v_{n+1}\right| \leq\left|a_{1}\right| \cdot \sum_{i=1}^{n}\left|v_{i}\right| \leq \frac{\epsilon}{1-n \epsilon} \cdot n(1+\epsilon)<1-\epsilon,
$$

which is the contradiction to the assumption. 
Proposition 6.6. For complete Riemannian manifold $\left(M^{n}, g\right)$ with $R c \geq 0$, if there are harmonic functions $\left\{\mathbf{b}_{i}\right\}_{i=1}^{n}$, defined on $B_{r}(p)$, satisfying $\mathbf{b}_{i}(p)=0$ and

$$
\sup _{\substack{B_{r}(p) \\ i=1, \cdots, n}}\left|\nabla \mathbf{b}_{i}\right| \leq 2, \quad \sup _{s \leq r} f_{B_{s}(p)} \sum_{i, j=1}^{n}\left|\left\langle\nabla \mathbf{b}_{i}, \nabla \mathbf{b}_{j}\right\rangle-\delta_{i j}\right| \leq n^{-80000 n^{7}},
$$

then $\Gamma_{\tilde{p}}\left(\frac{r}{12800}\right)=\{e\}$.

Proof: Let $\delta=n^{-80000 n^{7}}$ in the rest proof. From (6.17) and Lemma 6.2, let $\tilde{\mathbf{b}}_{i}=\mathcal{L}\left(\mathbf{b}_{i}\right)$, then $\tilde{\mathbf{b}}_{i}(\tilde{p})=\mathbf{b}_{i}(p)=0$ and

$$
\sup _{\substack{B_{r}(\tilde{\tilde{w}}) \\ i=1, \cdots, n}}\left|\nabla \tilde{\mathbf{b}}_{i}\right| \leq 2, \quad \quad \sup _{s \leq r} f_{B_{s}(\tilde{p})} \sum_{i, j=1}^{n}\left|\left\langle\nabla \tilde{\mathbf{b}}_{i}, \nabla \tilde{\mathbf{b}}_{j}\right\rangle-\delta_{i j}\right| \leq 4^{n} \delta .
$$

From (6.17), 6.18) and Theorem 2.13, if $\delta_{1}>0$ is to be determined later such that

$$
\delta \leq 4^{-n} \cdot n^{-700 n^{4}} \delta_{1}^{18 n^{4}},
$$

then there are two family of $\left(\delta_{1} \cdot s\right)$-Gromov-Hausdorff approximation for any $0<$ $s \leq 10 r_{c}$,

$$
\begin{aligned}
f_{s} & =\left(\mathbf{b}_{1}, \cdots, \mathbf{b}_{n}, \mathcal{P}_{s}\right): B_{s}(p) \rightarrow B_{s}\left(0, \hat{p}_{s}\right) \subset \mathbb{R}^{n} \times \mathbf{X}_{n, s} \\
\tilde{f}_{s} & =\left(\tilde{\mathbf{b}}_{1}, \cdots, \tilde{\mathbf{b}}_{n}, \tilde{\mathcal{P}}_{s}\right): B_{s}(\tilde{p}) \rightarrow B_{s}\left(0, \check{p}_{s}\right) \subset \mathbb{R}^{n} \times \tilde{\mathbf{X}}_{n, s},
\end{aligned}
$$

where $r_{c}=\frac{1}{12800} r$.

For any $0<t \leq r_{c}$, let $\operatorname{diam}\left(B_{t}\left(\hat{p}_{10 t}\right)\right)=t_{1}$. If $t_{1} \geq \frac{1}{4} t$, note

$$
f_{10 t}=\left(\mathbf{b}_{1}, \cdots, \mathbf{b}_{n}, \mathcal{P}_{10 t}\right): B_{10 t}(p) \rightarrow B_{10 t}\left(0, \hat{p}_{10 t}\right) \subset \mathbb{R}^{n} \times \mathbf{X}_{n, 10 t},
$$

is an $\left(10 \delta_{1} t\right)$-Gromov-Hausdorff approximation.

For $\epsilon>0$ to be determined later, assume

$$
\delta_{1}=n^{-3400 n^{3}} \epsilon^{110 n} .
$$

Apply Theorem 2.12, there exist harmonic functions $\left\{\mathbf{b}_{i}\right\}_{i=1}^{n+1}$ defined on $B_{l_{1}}(q) \subset$ $B_{10 t}(p)$ such that

$$
\sup _{\substack{B_{l^{\prime}}(q) \\ i=1, \cdots, n+1}}\left|\nabla \mathbf{b}_{i}\right| \leq 2 \quad \text { and } \quad \sup _{l \leq l_{1}} f_{B_{l}(q)} \sum_{i, j=1}^{n+1}\left|\left\langle\nabla \mathbf{b}_{i}, \nabla \mathbf{b}_{j}\right\rangle-\delta_{i j}\right| \leq \epsilon,
$$

where $l_{1}=n^{-320 n^{3}} \epsilon^{10 n} t$.

Let $l \rightarrow 0$ in the above, we get $\left\{v_{i}\right\}_{i=1}^{n+1}$, where $v_{i} \in T_{q} M^{n}$ such that

$$
\sup _{1 \leq i<j \leq n+1}\left|\left\langle v_{i}, v_{j}\right\rangle\right| \leq \epsilon \quad \text { and } \quad \sup _{1 \leq i \leq n+1}|| v_{i}|-1| \leq \epsilon .
$$

Choose suitable $\epsilon=\frac{1}{20 n^{2}}$, then (6.19) holds because of (6.20) and the definition of $\delta$.

Note $\epsilon<\frac{1}{10 n^{2}}$, from Lemma 6.5 it is impossible. Hence we have

$$
\operatorname{diam}\left(B_{t}\left(\hat{p}_{10 t}\right)\right)<\frac{1}{4} t, \quad \forall t \in\left(0, r_{c}\right] .
$$


Note the definition of $\delta$ implies

$$
\delta \leq n^{-900 n^{4}} 4^{-40 n^{4}} .
$$

For any $0<s \leq r_{c}$, from 6.22), 6.17, (6.21) and Lemma 6.4, we have

$$
\Gamma(s) \subset\left\langle\Gamma\left(4^{-1} s+2 \cdot \frac{1}{4} s\right)\right\rangle \subset\left\langle\Gamma\left(\frac{3}{4} s\right)\right\rangle, \quad \forall 0<s \leq r_{c} .
$$

From (6.23), by induction on $s$, for any positive integer $m$, we have

$$
\left\langle\Gamma_{\tilde{p}}\left(r_{c}\right)\right\rangle=\left\langle\Gamma_{\tilde{p}}\left(\frac{3}{4} r_{c}\right)\right\rangle=\cdots=\left\langle\Gamma_{\tilde{p}}\left(\left(\frac{3}{4}\right)^{m} r_{c}\right)\right\rangle .
$$

Note the group action is discrete, hence for big enough $m$, we have $\left\langle\Gamma_{\tilde{p}}\left(\left(\frac{3}{4}\right)^{m} r_{c}\right)\right\rangle=$ $\{e\}$, which implies $\left\langle\Gamma_{\tilde{p}}\left(r_{c}\right)\right\rangle=\{e\}$.

We define $\mathfrak{n g}\langle\Gamma(r)\rangle$ as the minimal number of generators in $\Gamma(r)$ needed to generated $\langle\Gamma(r)\rangle$.

Proposition 6.7. For $\tilde{p}, \tilde{q} \in\left(\tilde{M}^{n}, \tilde{g}\right)$ with $\operatorname{Rc}(\tilde{g}) \geq 0$, assume $d(\tilde{p}, \tilde{q}) \leq \delta<\frac{s}{2}$, then

$$
\begin{array}{ll}
\mathfrak{n g}\left\langle\Gamma_{\tilde{p}}\left(\lambda_{2}\right)\right\rangle \leq\left(\frac{2 \lambda_{2}+\lambda_{1}}{\lambda_{1}}\right)^{n} \mathfrak{n g}\left\langle\Gamma_{\tilde{p}}\left(\lambda_{1}\right)\right\rangle, & \text { if } 0<\lambda_{1} \leq \lambda_{2} \\
\mathfrak{n g}\left\langle\Gamma_{\tilde{p}}(s)\right\rangle \leq\left(\frac{3 s+2 \delta}{s-2 \delta}\right)^{n} \cdot \mathfrak{n g}\left\langle\Gamma_{\tilde{q}}(s-2 \delta)\right\rangle . &
\end{array}
$$

Proof: Note $\Gamma_{\tilde{p}}\left(\lambda_{2}\right)$ is a finite set, then there are only finite number of subsets $\mathcal{B}_{1}, \cdots, \mathcal{B}_{m} \subseteq \Gamma_{\tilde{p}}\left(\lambda_{2}\right)$ satisfying

$\{e\} \subseteq \mathcal{B}_{i} \quad$ and $\quad\left\{h_{1}^{-1} h_{2}: h_{1} \neq h_{2} \in \mathcal{B}_{i}\right\} \in \Gamma_{\tilde{p}}\left(\lambda_{2}\right)-\Gamma_{\tilde{p}}\left(\lambda_{1}\right) \quad \forall 1 \leq i \leq m$.

Let $\mathcal{A}_{\lambda_{1}, \lambda_{2}}$ be one of the above subsets, such that there does not exist $\mathcal{B}_{i}$ with $\mathcal{A}_{\lambda_{1}, \lambda_{2}} \varsubsetneqq \mathcal{B}_{i}$. Then $\mathcal{A}_{\lambda_{1}, \lambda_{2}} \subset \Gamma_{\tilde{p}}\left(\lambda_{2}\right)$ satisfy:

$$
d\left(\tilde{p}, h_{1}^{-1} h_{2} \tilde{p}\right) \geq \lambda_{1} \quad \text { if } h_{1} \neq h_{2}, h_{1}, h_{2} \in \mathcal{A}_{\lambda_{1}, \lambda_{2}} .
$$

And for any $\gamma \in \Gamma_{\tilde{p}}\left(\lambda_{2}\right)$, there is $h \in \mathcal{A}_{\lambda_{1}, \lambda_{2}}$ such that $h^{-1} \gamma \in \Gamma_{\tilde{p}}\left(\lambda_{1}\right)$.

For any $h \in \mathcal{A}_{\lambda_{1}, \lambda_{2}}$, we get $h \tilde{p} \in B_{\lambda_{2}}(\tilde{p})$. From (6.24),

$$
B_{\frac{\lambda_{1}}{2}}\left(g_{i} \tilde{p}\right) \cap B_{\frac{\lambda_{1}}{2}}\left(g_{j} \tilde{p}\right)=\emptyset \quad \text { if } g_{1} \neq g_{2}, g_{1}, g_{2} \in \mathcal{A}_{\lambda_{1}, \lambda_{2}} .
$$

Now let $\#\left(\mathcal{A}_{\lambda_{1}, \lambda_{2}}\right)$ denote the number of the elements in $\mathcal{A}_{\lambda_{1}, \lambda_{2}}$, we have

$$
\begin{aligned}
V\left(B_{\lambda_{2}+\frac{\lambda_{1}}{2}}(\tilde{p})\right) & \geq V\left(\bigcup_{g_{i} \in \mathcal{A}_{\lambda_{1}, \lambda_{2}}} B_{\frac{\lambda_{1}}{2}}\left(g_{i} \tilde{p}\right)\right)=\sum_{g_{i} \in \mathcal{A}_{\lambda_{1}, \lambda_{2}}} V\left(B_{\frac{\lambda_{1}}{2}}\left(g_{i} \tilde{p}\right)\right) \\
& =\sum_{g_{i} \in \mathcal{A}_{\lambda_{1}, \lambda_{2}}} V\left(B_{\lambda_{2}+\frac{\lambda_{1}}{2}}(\tilde{p})\right) \cdot\left[\frac{V\left(B_{\frac{\lambda_{1}}{2}}(\tilde{p})\right)}{V\left(B_{\lambda_{2}+\frac{\lambda_{1}}{2}}(\tilde{p})\right)}\right] \\
& \geq \#\left(\mathcal{A}_{\lambda_{1}, \lambda_{2}}\right) \cdot\left(\frac{\frac{\lambda_{1}}{2}}{\lambda_{2}+\frac{\lambda_{1}}{2}}\right)^{n} \cdot V\left(B_{\lambda_{2}+\frac{\lambda_{1}}{2}}(\tilde{p})\right) .
\end{aligned}
$$

In the last inequality the Bishop-Gromov Comparison Theorem is used. Then

$$
\#\left(\mathcal{A}_{\lambda_{1}, \lambda_{2}}\right) \leq\left(\frac{2 \lambda_{2}+\lambda_{1}}{\lambda_{1}}\right)^{n} .
$$


It is easy to see $\Gamma_{\tilde{p}}\left(\lambda_{2}\right) \subset \mathcal{A}_{\lambda_{1}, \lambda_{2}} \cdot \Gamma_{\tilde{p}}\left(\lambda_{1}\right)$, from (6.25), we have

$$
\mathfrak{n g}\left\langle\Gamma_{\tilde{p}}\left(\lambda_{2}\right)\right\rangle \leq \#\left(\mathcal{A}_{\lambda_{1}, \lambda_{2}}\right) \cdot \mathfrak{n g}\left\langle\Gamma_{\tilde{p}}\left(\lambda_{1}\right)\right\rangle \leq\left(\frac{2 \lambda_{2}+\lambda_{1}}{\lambda_{1}}\right)^{n} \mathfrak{n g}\left\langle\Gamma_{\tilde{p}}\left(\lambda_{1}\right)\right\rangle .
$$

For $\gamma \in \Gamma_{\tilde{p}}(s)$, we have

$$
d(\gamma \tilde{q}, \tilde{q}) \leq d(\gamma \tilde{q}, \gamma \tilde{p})+d(\gamma \tilde{p}, \tilde{p})+d(\tilde{p}, \tilde{q}) \leq 2 d(\tilde{p}, \tilde{q})+d(\gamma \tilde{p}, \tilde{p}) \leq s+2 \delta,
$$

which implies $\Gamma_{\tilde{p}}(s) \subset \Gamma_{\tilde{q}}(s+2 \delta)$. Then

$$
\begin{aligned}
\mathfrak{n g}\left\langle\Gamma_{\tilde{p}}(s)\right\rangle & \leq \mathfrak{n g}\left\langle\Gamma_{\tilde{q}}(s+2 \delta)\right\rangle \leq\left[\frac{2(s+2 \delta)+(s-2 \delta)}{s-2 \delta}\right]^{n} \cdot \mathfrak{n g}\left\langle\Gamma_{\tilde{q}}(s-2 \delta)\right\rangle \\
& =\left(\frac{3 s+2 \delta}{s-2 \delta}\right)^{n} \cdot \mathfrak{n g}\left\langle\Gamma_{\tilde{q}}(s-2 \delta)\right\rangle .
\end{aligned}
$$

Proposition 6.8. For any $\epsilon \in(0,1)$ and $0 \leq k<n$, there exists $\delta=\left(n^{-30} \epsilon\right)^{5000 n^{7}}$, such that if there exist harmonic functions $\left\{\mathbf{b}_{i}\right\}_{i=1}^{k}$, satisfying

$$
\sup _{\substack{B_{r}(q) \\ i=1, \cdots, k}}\left|\nabla \mathbf{b}_{i}\right| \leq 2 \quad \text { and } \quad \sup _{s \leq r} f_{B_{s}(q)} \sum_{i, j=1}^{k}\left|\left\langle\nabla \mathbf{b}_{i}, \nabla \mathbf{b}_{j}\right\rangle-\delta_{i j}\right| \leq \delta .
$$

Then there are harmonic functions $\left\{\mathbf{b}_{i}\right\}_{i=1}^{k+1}$ defined on $B_{r_{1}}\left(q_{1}\right) \subset B_{r}(q)$, such that

$$
\begin{aligned}
& \mathfrak{n g}\left\langle\Gamma_{\tilde{q}}(r)\right\rangle \leq n^{340 n^{4}} \epsilon^{-10 n^{2}} \cdot \mathfrak{n g}\left\langle\Gamma_{\tilde{q}_{1}}\left(r_{1}\right)\right\rangle \\
& \sup _{\substack{B_{r_{1}}\left(q_{1}\right) \\
i=1, \cdots, k+1}}\left|\nabla \mathbf{b}_{i}\right| \leq 2 \quad \text { and } \quad \sup _{t \leq r_{1}} f_{B_{t}\left(q_{1}\right)} \sum_{i, j=1}^{k+1}\left|\left\langle\nabla \mathbf{b}_{i}, \nabla \mathbf{b}_{j}\right\rangle-\delta_{i j}\right| \leq \epsilon .
\end{aligned}
$$

Proof: Set $r_{c}=\frac{1}{12800} r$, from Proposition 6.7,

$$
\mathfrak{n g}\left\langle\Gamma_{\tilde{q}}(r)\right\rangle \leq\left(\frac{2 r+r_{c}}{r_{c}}\right)^{n} \cdot \mathfrak{n g}\left\langle\Gamma_{\tilde{q}}\left(r_{c}\right)\right\rangle .
$$

Define $\hat{r}_{1}=\min \left\{s \geq 0 \mid \Gamma_{\tilde{q}}\left(r_{c}\right) \subset\left\langle\Gamma_{\tilde{q}}(s)\right\rangle\right\}$, then $\hat{r}_{1} \leq r_{c}$ and

$$
\Gamma_{\tilde{q}}\left(r_{c}\right) \subset\left\langle\Gamma_{\tilde{q}}\left(\hat{r}_{1}\right)\right\rangle .
$$

For $\delta_{1}>0$ to be determined later, we choose $\delta \leq n^{-900 n^{4}} \delta_{1}^{40 n^{4}}$. From (6.26) and apply Lemma 6.4 on $B_{10 \hat{r}_{1}}(q)$, we have a family of $\left(\delta_{1} \cdot s\right)$-Gromov-Hausdorff approximation for any $0<s \leq 10 \hat{r}_{1}$,

$$
f_{s}: B_{s}(q) \rightarrow B_{s}\left(0, \hat{q}_{s}\right) \subset \mathbb{R}^{k} \times \mathbf{X}_{k, s} .
$$

And assume $\operatorname{diam}\left(B_{\hat{r}_{1}}\left(\hat{q}_{10 \hat{r}_{1}}\right)\right)=r_{0}$, we have

$$
\Gamma_{\tilde{q}}\left(\hat{r}_{1}\right) \subset\left\langle\Gamma_{\tilde{q}}\left(\delta_{1} \hat{r}_{1}+2 r_{0}\right)\right\rangle .
$$

From (6.29) and 6.31), we have $\Gamma_{\tilde{q}}\left(r_{c}\right) \subset\left\langle\Gamma_{\tilde{q}}\left(\delta_{1} \hat{r}_{1}+2 r_{0}\right)\right\rangle$. Then by the definition of $\hat{r}_{1}$, we get $\hat{r}_{1} \leq \delta_{1} \hat{r}_{1}+2 r_{0}$. If we assume $\delta_{1} \leq \frac{1}{32}$, it yields $r_{0} \geq \frac{1}{2} \hat{r}_{1}-\frac{1}{2} \delta_{1} \hat{r}_{1} \geq \frac{1}{4} \hat{r}_{1}$. Note $\operatorname{diam}\left(B_{\hat{r}_{1}}\left(\hat{q}_{10 \hat{r}_{1}}\right)\right)=r_{0}$, then combining (6.30), choose $\delta_{1}=n^{-3400 n^{3}} \epsilon^{110 n}$. 
Apply Theorem 2.12, we can find harmonic functions $\left\{\mathbf{b}_{i}\right\}_{i=1}^{k+1}$ defined on $B_{r_{1}}\left(q_{1}\right) \subset$ $B_{10 \hat{r}_{1}}(q)$, such that

$$
\sup _{\substack{B_{r_{1}\left(q_{1}\right)} \\ i=1, \cdots, k+1}}\left|\nabla \mathbf{b}_{i}\right| \leq 2 \quad \text { and } \quad \sup _{t \leq r_{1}} f_{B_{t}\left(q_{1}\right)} \sum_{i, j=1}^{k+1}\left|\left\langle\nabla \mathbf{b}_{i}, \nabla \mathbf{b}_{j}\right\rangle-\delta_{i j}\right| \leq \epsilon,
$$

where $r_{1}=n^{-320 n^{3}} \epsilon^{10 n} \hat{r}_{1}>0$.

From Proposition 6.7 and $q_{1} \in B_{10 \hat{r}_{1}}(q)$, we have

$$
\begin{aligned}
\mathfrak{n g}\left\langle\Gamma_{\tilde{q}}\left(21 \hat{r}_{1}\right)\right\rangle & \leq(83)^{n} \mathfrak{n g}\left\langle\Gamma_{\tilde{q}_{1}}\left(\hat{r}_{1}\right)\right\rangle \leq(83)^{n} \cdot\left(\frac{2 \hat{r}_{1}+r_{1}}{r_{1}}\right)^{n} \cdot \mathfrak{n g}\left\langle\Gamma_{\tilde{q}_{1}}\left(r_{1}\right)\right\rangle \\
& \leq n^{330 n^{4}} \epsilon^{-10 n^{2}} \cdot \mathfrak{n g}\left\langle\Gamma_{\tilde{q}_{1}}\left(r_{1}\right)\right\rangle .
\end{aligned}
$$

From (6.28), (6.29) and (6.32), we have

$$
\mathfrak{n g}\left\langle\Gamma_{\tilde{q}}(r)\right\rangle \leq\left(\frac{2 r+r_{c}}{r_{c}}\right)^{n} \cdot \mathfrak{n g}\left\langle\Gamma_{\tilde{q}}\left(\hat{r}_{1}\right)\right\rangle \leq n^{340 n^{4}} \epsilon^{-10 n^{2}} \mathfrak{n g}\left\langle\Gamma_{\tilde{q}_{1}}\left(r_{1}\right)\right\rangle .
$$

Theorem 6.9. Assume $\varphi: \tilde{M}^{n} \rightarrow M^{n}$ is the covering map with covering group $\Gamma$ such that $M^{n}=\tilde{M}^{n} / \Gamma$, where $\left(\tilde{M}^{n}, \tilde{g}\right)$ and $\left(M^{n}, g\right)$ are two complete Riemannian manifolds and the metric $g$ is the quotient metric of $\tilde{g}$ with respect to group action of $\Gamma$, furthermore $R c \geq 0$, then $\mathfrak{n g}\left\langle\Gamma_{\tilde{p}}(1)\right\rangle \leq n^{n^{20 n}}$ for any $\tilde{p} \in \tilde{M}^{n}$.

Proof: Apply Proposition 6.8, firstly when $k=0$, we get

$$
\begin{aligned}
& \mathfrak{n g}\left\langle\Gamma_{\tilde{p}}(1)\right\rangle \leq n^{340 n^{4}} \epsilon_{1}^{-10 n^{2}} \cdot \mathfrak{n g}\left\langle\Gamma_{\tilde{q}_{1}}\left(r_{1}\right)\right\rangle \\
& \sup _{\substack{B_{r_{1}}\left(q_{1}\right) \\
i=1}}\left|\nabla \mathbf{b}_{1}\right| \leq 2 \quad \text { and }\left.\quad \sup _{t \leq r_{1}} f_{B_{t}\left(q_{1}\right)}|| \nabla \mathbf{b}_{1}\right|^{2}-1 \mid \leq \epsilon_{1},
\end{aligned}
$$

where $\epsilon_{1}=\left(n^{-30} \epsilon_{2}\right)^{\tau}, \tau:=5000 n^{7}$ and $\epsilon_{2}$ is to be determined later.

By induction, apply Proposition 6.8 , for $0 \leq k \leq(n-1)$, we get

$$
\begin{aligned}
& \mathfrak{n g}\left\langle\Gamma_{\tilde{p}}(1)\right\rangle \leq n^{340 n^{4}} \epsilon_{k+1}^{-10 n^{2}} \cdot \mathfrak{n g}\left\langle\Gamma_{\tilde{q}_{k+1}}\left(r_{k+1}\right)\right\rangle \\
& \sup _{\substack{B_{r_{k+1}}\left(q_{k+1}\right) \\
i=1, \cdots, k+1}}\left|\nabla \mathbf{b}_{i}\right| \leq 2, \quad \sup _{t \leq r_{k+1}} f_{B_{t}\left(q_{k+1}\right)} \sum_{i, j=1}^{k+1}\left|\left\langle\nabla \mathbf{b}_{i}, \nabla \mathbf{b}_{j}\right\rangle-\delta_{i j}\right| \leq \epsilon_{k+1} .
\end{aligned}
$$

Let $\epsilon_{j}=\left(n^{-30} \epsilon_{j+1}\right)^{\tau}, j=1, \cdots, n-1$.

We let $\epsilon_{n}=n^{-200 \tau}$, then from (6.33) and (6.34) for $k=(n-1)$, apply Proposition 6.6, we have $\Gamma_{\tilde{q}_{n}}\left(\frac{r_{n}}{12800}\right)=\{e\}$.

From the induction formula for $\epsilon_{j}$, we have

$$
\epsilon_{j}=n^{-30 \tau \sum_{i=0}^{n-j-1} \tau^{i}}\left(\epsilon_{n}\right)^{\tau^{n-j}}, \quad 1 \leq j \leq(n-1) .
$$


Then from (6.33), 6.35) and Proposition 6.7yields

$$
\begin{aligned}
\mathfrak{n g}\left\langle\Gamma_{\tilde{p}}(1)\right\rangle & \leq n^{340 n^{4}} \epsilon_{1}^{-10 n^{2}} \mathfrak{n g}\left\langle\Gamma_{\tilde{q}_{1}}\left(r_{1}\right)\right\rangle \\
& \leq\left(n^{340 n^{4}}\right)^{n} \cdot\left(\epsilon_{1} \cdots \epsilon_{n}\right)^{-10 n^{2}} \mathfrak{n g}\left\langle\Gamma_{\tilde{q}_{n}}\left(r_{n}\right)\right\rangle \\
& \leq n^{340 n^{5}} \cdot n^{1200 n^{2} \tau^{n-1}} \cdot \epsilon_{n}^{-20 n^{2} \tau^{n-1}} \cdot \mathfrak{n g}\left\langle\Gamma_{\tilde{q}_{n}}\left(r_{n}\right)\right\rangle \\
& \leq n^{5000 n^{2} \tau^{n}} \cdot\left(\frac{2 r_{n}+\frac{1}{12800} r_{n}}{\frac{1}{12800} r_{n}}\right) \mathfrak{n g}\left\langle\Gamma_{\tilde{q}_{n}}\left(\frac{1}{12800} r_{n}\right)\right\rangle \\
& \leq n^{2^{20 n}} .
\end{aligned}
$$

Theorem 6.10. Suppose $\left(M^{n}, g\right)$ is a complete Riemannian manifold with $R c \geq 0$, then for any finitely generated subgroup $\Gamma$ of $\pi_{1}\left(M^{n}\right)$, we have $\mathfrak{n g}(\Gamma) \leq n^{n^{20 n}}$.

Proof: From [JRM00, Theorem 82.1], there exists a covering map $\varphi: N^{n} \rightarrow M^{n}$ such that $\varphi_{*}\left(\pi_{1}\left(N^{n}, \hat{p}\right)\right)=\Gamma$, where $\varphi(\hat{p})=p$. Now from [JRM00, Theorem 54.6(a)], we get $\pi_{1}\left(N^{n}, \hat{p}\right) \simeq \Gamma$. Hence there exists a complete Riemannian manifold $\left(N^{n}, \tilde{g}\right)$ with $R c\left(N^{n}\right) \geq 0$ and $\pi_{1}\left(N^{n}\right)=\Gamma$.

Assume $\Gamma=\left\langle\gamma_{1}, \cdots, \gamma_{k}\right\rangle$ and $\sup _{i=1, \cdots, k} d\left(\gamma_{i} \hat{p}, \hat{p}\right) \leq C_{1}$. Then by scaling the metric $\tilde{g}$ to $\hat{g}=C_{1}^{-1} \tilde{g}$, we get that $\Gamma=\left\langle\Gamma_{\hat{p}}(1)\right\rangle$, where $\hat{p} \in\left(N^{n}, \hat{g}\right)$. From Theorem 6.9, we have $\mathfrak{n g}(\Gamma) \leq \mathfrak{n g}\left\langle\Gamma_{\hat{p}}(1)\right\rangle \leq n^{n^{20 n}}$.

\section{ACKNOWLEDGMENTS}

The author thank Aaron Naber for helpful suggestion, which greatly improves the quantitative estimates in the earlier version of this paper. We are grateful to Jiaping Wang for continuous encouragement, William P. Minicozzi II and Christina Sormani for comments. We are indebted to Vitali Kapovitch for several email reply about [KW11], which help us to understand the results there better.

\section{REFERENCES}

[AG90] Uwe Abresch and Detlef Gromoll, On complete manifolds with nonnegative Ricci curvature, J. Amer. Math. Soc. 3 (1990), no. 2, 355-374, DOI 10.2307/1990957.

[And90] Michael T. Anderson, On the topology of complete manifolds of nonnegative Ricci curvature, Topology 29 (1990), no. 1, 41-55, DOI 10.1016/0040-9383(90)90024-E.

[Arn89] V. I. Arnol'd, Mathematical methods of classical mechanics, Second, Graduate Texts in Mathematics, vol. 60, Springer-Verlag, New York, 1989. Translated from the Russian by K. Vogtmann and A. Weinstein.

[BGS85] Werner Ballmann, Mikhael Gromov, and Viktor Schroeder, Manifolds of nonpositive curvature, Progress in Mathematics, vol. 61, Birkhäuser Boston, Inc., Boston, MA, 1985.

[Che99] J. Cheeger, Differentiability of Lipschitz functions on metric measure spaces, Geom. Funct. Anal. 9 (1999), no. 3, 428-517, DOI 10.1007/s000390050094.

[CC96] Jeff Cheeger and Tobias H. Colding, Lower bounds on Ricci curvature and the almost rigidity of warped products, Ann. of Math. (2) 144 (1996), no. 1, 189-237, DOI $10.2307 / 2118589$.

[CC97] _ On the structure of spaces with Ricci curvature bounded below. I, J. Differential Geom. 46 (1997), no. 3, 406-480, MR1484888, Zbl 0902.53034. 
[CC00a] On the structure of spaces with Ricci curvature bounded below. II, J. Differential Geom. 54 (2000), no. 1, 13-35.

[CCOOb] On the structure of spaces with Ricci curvature bounded below. III, J. Differential Geom. 54 (2000), no. 1, 37-74.

[CG71] Jeff Cheeger and Detlef Gromoll, The splitting theorem for manifolds of nonnegative Ricci curvature, J. Differential Geometry 6 (1971/72), 119-128.

[CG72] _ On the structure of complete manifolds of nonnegative curvature, Ann. of Math. (2) 96 (1972), 413-443.

[CN15] Jeff Cheeger and Aaron Naber, Regularity of Einstein manifolds and the codimension 4 conjecture, Ann. of Math. (2) 182 (2015), no. 3, 1093-1165, DOI 10.4007/annals.2015.182.3.5.

[CY75] S. Y. Cheng and S. T. Yau, Differential equations on Riemannian manifolds and their geometric applications, Comm. Pure Appl. Math. 28 (1975), no. 3, 333-354.

[Col96a] Tobias H. Colding, Shape of manifolds with positive Ricci curvature, Invent. Math. 124 (1996), no. 1-3, 175-191, DOI 10.1007/s002220050049.

[Col96b] _ Large manifolds with positive Ricci curvature, Invent. Math. 124 (1996), no. 1-3, 193-214, DOI 10.1007/s002220050050.

[Co197] _ Ricci curvature and volume convergence, Ann. of Math. (2) 145 (1997), no. 3, 477-501, DOI 10.2307/2951841.

[CMI14] Tobias Holck Colding and William P. Minicozzi II, On uniqueness of tangent cones for Einstein manifolds, Invent. Math. 196 (2014), no. 3, 515-588, DOI 10.1007/s00222-0130474-z.

[CN12] Tobias Holck Colding and Aaron Naber, Sharp Hölder continuity of tangent cones for spaces with a lower Ricci curvature bound and applications, Ann. of Math. (2) 176 (2012), no. 2, 1173-1229, DOI 10.4007/annals.2012.176.2.10.

[Fuk86] Kenji Fukaya, Theory of convergence for Riemannian orbifolds, Japan. J. Math. (N.S.) 12 (1986), no. 1, 121-160.

[FY92] Kenji Fukaya and Takao Yamaguchi, The fundamental groups of almost non-negatively curved manifolds, Ann. of Math. (2) 136 (1992), no. 2, 253-333, DOI 10.2307/2946606.

[GT01] David Gilbarg and Neil S. Trudinger, Elliptic partial differential equations of second order, Reprint of the 1998 edition. Classics in Mathematics. Springer-Verlag, Berlin, xiv +517 pp, 2001.

[Gro78] M. Gromov, Almost flat manifolds, J. Differential Geom. 13 (1978), no. 2, 231-241.

[Gro99] Misha Gromov, Metric structures for Riemannian and non-Riemannian spaces, Progress in Mathematics, vol. 152, Birkhäuser Boston, Inc., Boston, MA, 1999. Based on the 1981 French original [ MR0682063 (85e:53051)], With appendices by M. Katz, P. Pansu and S. Semmes, Translated from the French by Sean Michael Bates.

[KW11] Vitali Kapovitch and Burkhard Wilking, Structure of fundamental groups of manifolds with Ricci curvature bounded below, arXiv:1105.5955 [math.DG] (2011).

[Li86] Peter Li, Large time behavior of the heat equation on complete manifolds with nonnegative Ricci curvature, Ann. of Math. (2) 124 (1986), no. 1, 1-21, DOI 10.2307/1971385.

[LY86] Peter Li and Shing-Tung Yau, On the parabolic kernel of the Schrödinger operator, Acta Math. 156 (1986), no. 3-4, 153-201, DOI 10.1007/BF02399203.

[Mil68] J. Milnor, A note on curvature and fundamental group, J. Differential Geometry 2 (1968), $1-7$.

[JRM00] James R. Munkres, Topology, 2nd ed., Upper Saddle River, NJ, Prentice Hall, 2000.

[Rag72] M. S. Raghunathan, Discrete subgroups of Lie groups, Springer-Verlag, New YorkHeidelberg, 1972. Ergebnisse der Mathematik und ihrer Grenzgebiete, Band 68.

[Sor00] Christina Sormani, Nonnegative Ricci curvature, small linear diameter growth and finite generation of fundamental groups, J. Differential Geom. 54 (2000), no. 3, 547-559.

[Wei97] Guofang Wei, Ricci curvature and Betti numbers, J. Geom. Anal. 7 (1997), no. 3, 493-509, DOI 10.1007/BF02921630. 
[Wil00] Burkhard Wilking, On fundamental groups of manifolds of nonnegative curvature, Differential Geom. Appl. 13 (2000), no. 2, 129-165, DOI 10.1016/S0926-2245(00)00030-9.

Department of Mathematical Sciences,, Tsinghua University, Beijing, P. R. China, 100084

E-mail address: guoyixu@tsinghua. edu.cn 